\title{
CONVERGENCE ANALYSIS OF A LOCALLY STABILIZED COLLOCATED FINITE VOLUME SCHEME FOR INCOMPRESSIBLE FLOWS
}

\author{
Robert Eymard ${ }^{1}$, Raphà̀le Herbin ${ }^{2}$, Jean-Claude Latché $^{3}$ and Bruno Piar ${ }^{3}$
}

\begin{abstract}
We present and analyse in this paper a novel cell-centered collocated finite volume scheme for incompressible flows. Its definition involves a partition of the set of control volumes; each element of this partition is called a cluster and consists in a few neighbouring control volumes. Under a simple geometrical assumption for the clusters, we obtain that the pair of discrete spaces associating the classical cell-centered approximation for the velocities and cluster-wide constant pressures is inf-sup stable; in addition, we prove that a stabilization involving pressure jumps only across the internal edges of the clusters yields a stable scheme with the usual collocated discretization (i.e., in particular, with control-volume-wide constant pressures), for the Stokes and the Navier-Stokes problem. An analysis of this stabilized scheme yields the existence of the discrete solution (and uniqueness for the Stokes problem). The convergence of the approximate solution toward the solution to the continuous problem as the mesh size tends to zero is proven, provided, in particular, that the approximation of the mass balance flux is second order accurate; this condition imposes some geometrical conditions on the mesh. Under the same assumption, an error analysis is provided for the Stokes problem: it yields first-order estimates in energy norms. Numerical experiments confirm the theory and show, in addition, a second order convergence for the velocity in a discrete $\mathrm{L}^{2}$ norm.
\end{abstract}

Mathematics Subject Classification. 65N12, 65N15, 65N30, 76D05, 76D07, 76M25.

Received October 30, 2006.

Published online August 1st, 2009.

\section{INTRODUCTION}

The use of collocated finite volumes for fluid flow problems is appealing for several reasons. Among them, let us mention a very inexpensive assembling step (in particular compared to finite elements, because there is no numerical integration to perform), the possibility to use, at least to some extent, general unstructured meshes with a low complexity of the data structure (compared with staggered schemes) suitable for the implementation of adaptative mesh refinement strategies and, finally, an easy coupling with additional conservation laws solvers, when these latters are developed within the finite volume framework. These features make collocated finite volumes attractive for industrial problems, and they are widely used in Computational Fluid Dynamics,

Keywords and phrases. Finite volumes, collocated discretizations, Stokes problem, Navier-Stokes equations, incompressible flows, analysis.

1 Université de Marne-la-Vallée, France. robert. eymard@univ-mlv.fr

2 Université de Provence, France. herbin@cmi.univ-mrs.fr

${ }^{3}$ Institut de Radioprotection et de Sûreté Nucléaire (IRSN), France. jean-claude.latche@irsn.fr; bruno.piar@irsn.fr 
either in commercial (FLUENT, CFX, ...) or in proprietary codes, as encountered for instance in nuclear safety [1], which is a part of the context of this study.

When applied to incompressible flow problems, cell-centered collocated finite volumes suffer from a lack of coercivity, which was shown in $[13,15]$ to be cured by a stabilization similar to the Brezzi-Pitkäranta technique, well-known in the finite element context. When this stabilization is used, the existence of a solution to the discrete problem (unique in the linear case, i.e. the Stokes problem) is ensured, together with its convergence toward the solution to the continuous problem, in both the steady and unsteady cases; for the steady Stokes problem and particular meshes, first order error estimates in natural (energy) norms are given in [13].

However, at high Reynolds numbers, numerical experiments show that the Brezzi-Pitkäranta stabilization term necessary to avoid pressure oscillations severely reduces the accuracy of the solution. To overcome this problem, the so-called "Collocated Clustered Finite Volume scheme" was introduced in [5]. The idea of this scheme is to implement a stabilization designed to damp the short wavelengths oscillations of the pressure within a given cluster (i.e. a small group of control volumes), since the original equations are indeed sufficient to control the long wavelength ones. In fact, one could even imagine to consider pressures which are constant on the clusters, but this turns out to be (numerically) not so favorable in terms of accuracy; moreover, the principle of one pressure per control volume is easier to implement, as the pressure then shares the discretization of other variables, and thus the same computer data structures. Following these ideas, the cluster stabilization which was implemented in [5] consists in using a penalization of the pressure jumps only across the edges located within each cluster. This scheme gives very high quality results both for the Boussinesq approximation at high Reynolds numbers and the low Mach number approximation.

The goal of the present paper is to study the mathematical properties of this Collocated Clustered Finite Volume scheme. Concerning the stability issue, our results are two-fold: first, we prove that a simple geometrical property for the clusters is equivalent to the inf-sup stability (e.g. [19]) of the pair of approximation spaces obtained by combining the standard cell-centered approximation for the velocity and an approximation of the pressure piecewise constant over each cluster; then this property is shown to yield the stability of the scheme. Under the same additional regularity property of the mesh as in [13], which seems in practice rather restrictive, we prove, with the analysis tools of [11], the convergence of the velocity and the pressure toward the exact solution as the mesh size tends to 0, for both the steady Stokes and Navier-Stokes equations. In addition, we also obtain a first order error estimate in natural norms for the Stokes problem.

The paper is organized as follows: in Section 2, we present the considered continuous problems and the weak formulations which are used in the subsequent analysis. In Section 3, we define the discretization spaces and recall some fundamental results on the finite volume schemes. The Collocated Clustered Finite Volume scheme is then presented and analysed for the Stokes problem in Section 4, and for the Navier-Stokes equations in Section 5. Some numerical results are presented in Section 6.

\section{The Continuous problem}

We are interested in this paper in finding an approximation of the fields $\overline{\boldsymbol{u}}=\left(\overline{\boldsymbol{u}}^{(i)}\right)_{i=1, \ldots, d}: \Omega \rightarrow \mathbb{R}^{d}$, and $\bar{p}: \Omega \rightarrow \mathbb{R}$, weak solution to the generalized incompressible steady Navier-Stokes equations which read:

$$
\begin{array}{ll}
\eta \overline{\boldsymbol{u}}^{(i)}+\sum_{j=1}^{d} \overline{\boldsymbol{u}}^{(j)} \partial_{j} \overline{\boldsymbol{u}}^{(i)}-\nu \Delta \overline{\boldsymbol{u}}^{(i)}+\partial_{i} \bar{p}=\boldsymbol{f}^{(i)} & \text { in } \Omega, \text { for } i=1, \ldots, d \\
\operatorname{div} \overline{\boldsymbol{u}}=\sum_{i=1}^{d} \partial_{i} \overline{\boldsymbol{u}}^{(i)}=0 & \text { in } \Omega
\end{array}
$$

with a homogeneous Dirichlet boundary condition for $\overline{\boldsymbol{u}}$. In the above equations, $\overline{\boldsymbol{u}}^{(i)}, i=1, \ldots, d$ denote the components of the velocity of a fluid which flows in a domain $\Omega, \partial_{i}$ stands for the partial derivative with respect 
to the $i$ th variable, $\bar{p}$ denotes the pressure, $\nu>0$ stands for the viscosity of the fluid. In tensor form, the first equation of (2.1) equivalently reads:

$$
\eta \overline{\boldsymbol{u}}+(\overline{\boldsymbol{u}} \cdot \boldsymbol{\nabla}) \overline{\boldsymbol{u}}-\nu \Delta \overline{\boldsymbol{u}}+\nabla \bar{p}=\boldsymbol{f} .
$$

We make the following assumptions:

$\Omega$ is an open bounded connected subset of $\mathbb{R}^{d}$, supposed to polygonal $(d=2)$

or polyhedral $(d=3)$,

$\nu \in(0,+\infty), \eta \in[0,+\infty)$

$$
\boldsymbol{f} \in \mathrm{L}^{2}(\Omega)^{d} .
$$

We denote by $\boldsymbol{x}=\left(\boldsymbol{x}^{(i)}\right)_{i=1, \ldots, d}$ any point of $\Omega$, by $|\cdot|$ the Euclidean norm in $\mathbb{R}^{d}$, and by $\mathrm{d} \boldsymbol{x}$ the $d$-dimensional Lebesgue measure $\mathrm{d} \boldsymbol{x}=\mathrm{d} \boldsymbol{x}^{(1)} \ldots \mathrm{d} \boldsymbol{x}^{(d)}$.

The weak sense that we consider for the Navier-Stokes equations is the following one.

Definition 2.1 (weak solution to the steady Navier-Stokes equations). Under hypotheses $(2.2)-(2.4),(\overline{\boldsymbol{u}}, \bar{p})$ is called a weak solution to (2.1) with a homogeneous Dirichlet boundary condition if:

$$
\begin{array}{|ll}
\overline{\boldsymbol{u}} \in \mathrm{H}_{0}^{1}(\Omega)^{d}, \bar{p} \in \mathrm{L}^{2}(\Omega) \text { with } \int_{\Omega} \bar{p}(\boldsymbol{x}) \mathrm{d} \boldsymbol{x}=0 \text { and: } & \\
\eta \int_{\Omega} \overline{\boldsymbol{u}}(\boldsymbol{x}) \cdot \overline{\boldsymbol{v}}(\boldsymbol{x}) \mathrm{d} \boldsymbol{x}+\nu \int_{\Omega} \boldsymbol{\nabla} \overline{\boldsymbol{u}}(\boldsymbol{x}): \boldsymbol{\nabla} \overline{\boldsymbol{v}}(\boldsymbol{x}) \mathrm{d} \boldsymbol{x}+b(\overline{\boldsymbol{u}}, \overline{\boldsymbol{u}}, \overline{\boldsymbol{v}}) & \\
\quad-\int_{\Omega} \bar{p}(\boldsymbol{x}) \operatorname{div} \overline{\boldsymbol{v}}(\boldsymbol{x}) \mathrm{d} \boldsymbol{x}=\int_{\Omega} \boldsymbol{f}(\boldsymbol{x}) \cdot \overline{\boldsymbol{v}}(\boldsymbol{x}) \mathrm{d} \boldsymbol{x} & \forall \overline{\boldsymbol{v}} \in \mathrm{H}_{0}^{1}(\Omega)^{d} \\
\int_{\Omega} \bar{q}(\boldsymbol{x}) \operatorname{div} \overline{\boldsymbol{u}}(\boldsymbol{x}) \mathrm{d} \boldsymbol{x}=0 & \forall \bar{q} \in \mathrm{L}^{2}(\Omega),
\end{array}
$$

where, for all $\overline{\boldsymbol{u}}, \overline{\boldsymbol{v}} \in \mathrm{H}_{0}^{1}(\Omega)^{d}$ and for a.e. $\boldsymbol{x} \in \Omega$, we use the following notation:

$$
\boldsymbol{\nabla} \overline{\boldsymbol{u}}(\boldsymbol{x}): \boldsymbol{\nabla} \overline{\boldsymbol{v}}(\boldsymbol{x})=\sum_{i=1}^{d} \boldsymbol{\nabla} \overline{\boldsymbol{u}}^{(i)}(\boldsymbol{x}) \cdot \boldsymbol{\nabla} \overline{\boldsymbol{v}}^{(i)}(\boldsymbol{x})
$$

and where the trilinear form $b(., .,$.$) is defined, for all \overline{\boldsymbol{u}}, \overline{\boldsymbol{v}}, \overline{\boldsymbol{w}} \in \mathrm{H}_{0}^{1}(\Omega)^{d}$, by:

$$
b(\overline{\boldsymbol{u}}, \overline{\boldsymbol{v}}, \overline{\boldsymbol{w}})=\sum_{i=1}^{d} \sum_{j=1}^{d} \int_{\Omega} \overline{\boldsymbol{u}}^{(j)}(\boldsymbol{x}) \partial_{j} \overline{\boldsymbol{v}}^{(i)}(\boldsymbol{x}) \overline{\boldsymbol{w}}^{(i)}(\boldsymbol{x}) \mathrm{d} \boldsymbol{x}=\int_{\Omega}(\overline{\boldsymbol{u}} \cdot \boldsymbol{\nabla}) \overline{\boldsymbol{v}} \cdot \overline{\boldsymbol{w}} \mathrm{d} \boldsymbol{x} .
$$

The proof of existence of at least one solution to (2.5) can be found, for instance, in [23].

Before dealing with the Navier-Stokes equations, we shall first analyze a scheme for the related linear problem, namely the generalized Stokes equations with homogeneous Dirichlet boundary conditions, which read:

$$
\mid \begin{array}{ll}
\eta \overline{\boldsymbol{u}}-\nu \Delta \overline{\boldsymbol{u}}+\boldsymbol{\nabla} \bar{p}=\boldsymbol{f} & \text { in } \Omega \\
\operatorname{div} \overline{\boldsymbol{u}}=g & \text { in } \Omega
\end{array}
$$

where $g$ is a source term supposed to belong to $\mathrm{L}^{2}(\Omega)$ and to be such that $\int_{\Omega} g(\boldsymbol{x}) \mathrm{d} \boldsymbol{x}=0$. 
We then consider the following weak sense for this problem.

Definition 2.2 (weak solution to the steady Stokes equations). Under hypotheses $(2.2)-(2.4),(\overline{\boldsymbol{u}}, \bar{p})$ is called a weak solution to (2.7) if:

$$
\begin{aligned}
& \overline{\boldsymbol{u}} \in \mathrm{H}_{0}^{1}(\Omega)^{d}, \bar{p} \in \mathrm{L}^{2}(\Omega) \text { with } \int_{\Omega} \bar{p}(\boldsymbol{x}) \mathrm{d} \boldsymbol{x}=0 \text { and: } \\
& \eta \int_{\Omega} \overline{\boldsymbol{u}}(\boldsymbol{x}) \cdot \overline{\boldsymbol{v}}(\boldsymbol{x}) \mathrm{d} \boldsymbol{x}+\nu \int_{\Omega} \boldsymbol{\nabla} \overline{\boldsymbol{u}}(\boldsymbol{x}): \nabla \overline{\boldsymbol{v}}(\boldsymbol{x}) \mathrm{d} \boldsymbol{x}-\int_{\Omega} \bar{p}(\boldsymbol{x}) \operatorname{div} \overline{\boldsymbol{v}}(\boldsymbol{x}) \mathrm{d} \boldsymbol{x}=\int_{\Omega} \boldsymbol{f}(\boldsymbol{x}) \cdot \overline{\boldsymbol{v}}(\boldsymbol{x}) \mathrm{d} \boldsymbol{x} \quad \forall \overline{\boldsymbol{v}} \in \mathrm{H}_{0}^{1}(\Omega)^{d} \\
& \int_{\Omega} \bar{q}(\boldsymbol{x}) \operatorname{div} \overline{\boldsymbol{u}}(\boldsymbol{x}) \mathrm{d} \boldsymbol{x}=\int_{\Omega} g(\boldsymbol{x}) \bar{q}(\boldsymbol{x}) \mathrm{d} \boldsymbol{x} \quad \forall \bar{q} \in \mathrm{L}^{2}(\Omega) .
\end{aligned}
$$

The existence and uniqueness of the weak solution to (2.7) in the sense of the above definition is a classical result (e.g. [23] or [3]).

\section{Spatial Discretization}

\subsection{Discretization of the computational domain}

\subsubsection{Admissible and super-admissible discretizations}

We first enrich the definition of admissible discretization for a finite volume method given in [11] by introducing the notion of cluster. The first three items of the following definition are thus classical, and only the last one is new.

Definition 3.1 (admissible discretization). Let $\Omega$ be an open bounded $(d=2)$ or polyhedral $(d=3)$ subset of $\mathbb{R}^{d}$, and $\partial \Omega=\bar{\Omega} \backslash \Omega$ its boundary. An admissible finite volume discretization of $\Omega$, denoted by $\mathcal{D}$, is given by $\mathcal{D}=(\mathcal{M}, \mathcal{E}, \mathcal{P}, \mathcal{G})$, where:

$-\mathcal{M}$ is a finite family of non empty open polygonal $(d=2)$ or polyhedral $(d=3)$ convex disjoint subsets of $\Omega$ (the "control volumes") such that $\bar{\Omega}=\cup_{K \in \mathcal{M}} \bar{K}$. For any $K \in \mathcal{M}$, let $\partial K=\bar{K} \backslash K$ be the boundary of $K$.

- $\mathcal{E}$ is a finite family of disjoint subsets of $\bar{\Omega}$ (the "edges" of the mesh), such that, for all $\sigma \in \mathcal{E}$, there exists a hyperplane $E$ of $\mathbb{R}^{d}$ and $K \in \mathcal{M}$ with $\bar{\sigma}=\partial K \cap E$ and $\sigma$ is a non empty open subset of $E$. We assume that, for all $K \in \mathcal{M}$, there exists a subset $\mathcal{E}(K)$ of $\mathcal{E}$ such that $\partial K=\cup_{\sigma \in \mathcal{E}(K)} \bar{\sigma}$. It results from the previous hypotheses that, for all $\sigma \in \mathcal{E}$, either $\sigma \subset \partial \Omega$ or there exists $(K, L) \in \mathcal{M}^{2}$ with $K \neq L$ such that $\bar{K} \cap \bar{L}=\bar{\sigma}$; we denote in the latter case $\sigma=K \mid L$.

- $\mathcal{P}$ is a family of points of $\Omega$ indexed by $\mathcal{M}$, denoted by $\mathcal{P}=\left(\boldsymbol{x}_{K}\right)_{K \in \mathcal{M}}$. The family $\mathcal{P}$ is such that, for all $K \in \mathcal{M}, \boldsymbol{x}_{K} \in K$. Furthermore, for all $\sigma \in \mathcal{E}$ such that there exists $(K, L) \in \mathcal{M}^{2}$ with $\sigma=K \mid L$, it is assumed that the straight line $\left(\boldsymbol{x}_{K}, \boldsymbol{x}_{L}\right)$ going through $\boldsymbol{x}_{K}$ and $\boldsymbol{x}_{L}$ is orthogonal to $K \mid L$.

- $\mathcal{G}$ is a partition of $\mathcal{M}$ : the elements of $\mathcal{G}$ (the so-called clusters) are disjoint subsets of $\mathcal{M}$, the union of which is equal to $\mathcal{M}$.

The set of interior (resp. boundary) edges is denoted by $\mathcal{E}_{\text {int }}$ (resp. $\mathcal{E}_{\text {ext }}$ ), that is $\mathcal{E}_{\text {int }}=\{\sigma \in \mathcal{E} ; \sigma \not \subset \partial \Omega\}$ (resp. $\left.\mathcal{E}_{\text {ext }}=\{\sigma \in \mathcal{E} ; \sigma \subset \partial \Omega\}\right)$.

For all $K \in \mathcal{M}$, we denote by $\mathcal{N}_{K}$ the subset of $\mathcal{M}$ of the neighbouring control volumes to $K$ (i.e. the control volumes sharing an edge with $K$ ) excluding $K$ (i.e. $\left.K \notin \mathcal{N}_{K}\right)$ and by $G_{K}$ the unique element of $\mathcal{G}$ such that $K \in G_{K}$. Let us note that the property $\left(\forall K \in \mathcal{M}, \mathcal{N}_{K} \subset G_{K}\right)$ cannot hold, since $\left(G_{K}\right)_{K \in \mathcal{M}}$ is a partition of $\mathcal{M}$ while $\left(\mathcal{N}_{K}\right)_{K \in \mathcal{M}}$ is not. But, for any $K \in \mathcal{M}$, we suppose that $\mathcal{N}_{K} \cap G_{K} \neq \emptyset$.

Definition 3.2 (super-admissible discretization). An admissible discretization is said to be "super-admissible", if, for any internal $\sigma=K \mid L$, the line $\left(\boldsymbol{x}_{K}, \boldsymbol{x}_{L}\right)$ meets $K \mid L$ at its center of gravity. 


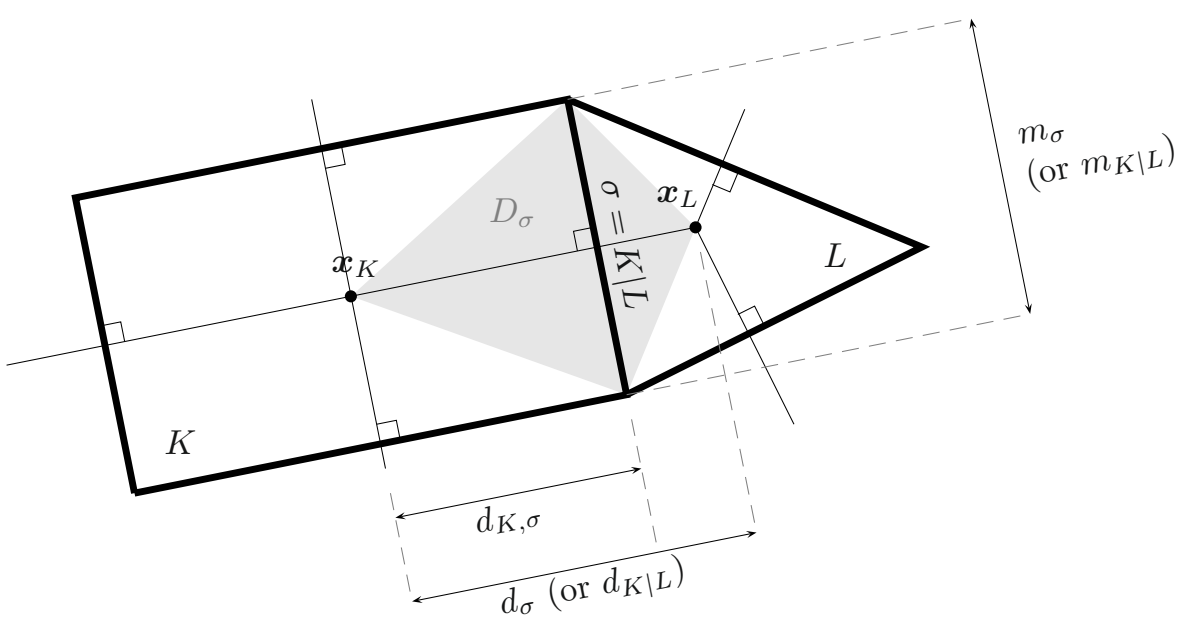

FiguRE 1. Notations for two neighbouring control volumes.

Typical super-admissible cells are rectangles and triangles with acute inner angles. An example of two such cells of a super-admissible mesh is given in Figure 1, along with some of the notations introduced in the next section.

\subsubsection{Geometrical quantities}

For each control volume $K \in \mathcal{M}$ and each edge $\sigma \in \mathcal{E}$ of the mesh, we denote by $m_{K}>0$ and $m_{\sigma}>0$ the measure of $K$ and the (d-1)-dimensional measure of $\sigma$, respectively. We denote by $d_{K, \sigma}$ the Euclidean distance between $\boldsymbol{x}_{K}$ and $\sigma$. For each internal edge of the mesh $\sigma=K \mid L, d_{\sigma}$ is defined as the Euclidean distance between $\boldsymbol{x}_{K}$ and $\boldsymbol{x}_{L}$; the notation is extended to any external edge $\sigma$ of a control volume $K$, for which we set $d_{\sigma}=d_{K, \sigma}$. For all $K \in \mathcal{M}$ and $L \in \mathcal{N}_{K}, \boldsymbol{n}_{K \mid L}$ stands for the unit vector normal to the edge $K \mid L$ outward to $K$ (so $\boldsymbol{n}_{L \mid K}=-\boldsymbol{n}_{K \mid L}$ ). To refer to a geometrical quantity related to any internal edge $\sigma=K \mid L$, we will use indifferently in this paper the index $\sigma$ or the index $K \mid L$ (i.e., for instance, $d_{\sigma}$ or $d_{K \mid L}, m_{\sigma}$ or $\left.m_{K \mid L} \ldots\right)$.

For each edge $\sigma$ of any control volume $K$, we denote by $D_{K, \sigma}$ the volume defined by:

$$
D_{K, \sigma}=\left\{t \boldsymbol{x}+(1-t) \boldsymbol{x}_{K}, \boldsymbol{x} \in \sigma, t \in(0,1)\right\}
$$

The so-called diamond-cell associated with the edge $\sigma$ is defined by $D_{\sigma}=D_{K, \sigma} \cup D_{L, \sigma}$ when $\sigma \in \mathcal{E}_{\text {int }}, \sigma=K \mid L$ and $D_{\sigma}=D_{K, \sigma}$ when $\sigma \in \mathcal{E}_{\text {ext }}, \sigma \in \mathcal{E}(K)$.

We define two parameters to characterize the size of the discretization, $h_{\mathcal{M}}$ and $h_{\mathcal{G}}$, as, respectively the maximal diameter of the control volumes and of the clusters:

$$
h_{\mathcal{M}}=\sup _{K \in \mathcal{M}} h_{K}, \quad h_{\mathcal{G}}=\sup _{G \in \mathcal{G}} h_{G}
$$

where $h_{K}$ and $h_{G}$ stand respectively for the diameter of the control volume $K$ and of the subset $\bigcup_{K \in G} K$ of $\mathbb{R}^{d}$. 

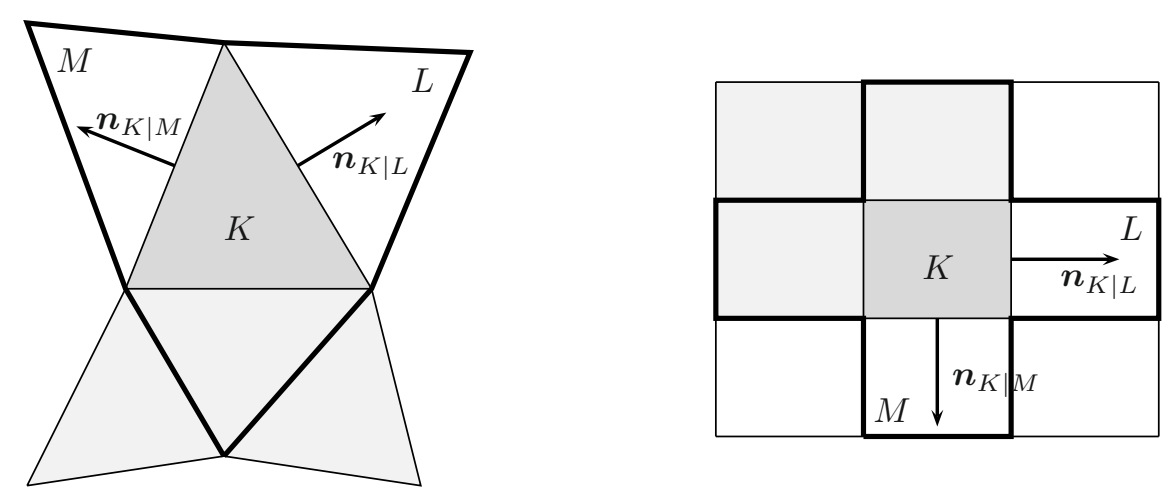

Admissible clusters:

$a_{L} \boldsymbol{n}_{K \mid L}+a_{M} \boldsymbol{n}_{K \mid M}=0 \quad \Rightarrow \quad a_{L}=a_{M}=0$

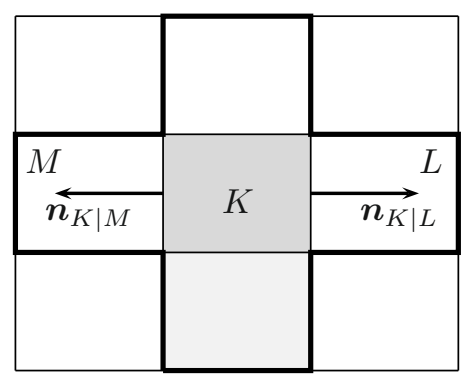

Non admissible cluster: $\quad \frac{\sqrt{2}}{2} \boldsymbol{n}_{K \mid L}+\frac{\sqrt{2}}{2} \boldsymbol{n}_{K \mid M}=0$

Figure 2. Admissible and non admissible clusters. For a given cell $K$ (in dark gray), the set of cells defining the cluster $G_{K}$ is colored in gray (and includes $K$ ), and the set $\mathcal{N}_{K}$ of neighbouring cells to $K$ is outlined with a bold line.

\subsubsection{Regularity of the mesh}

We assume that the clusters satisfy the following geometrical assumption:

$$
\forall K \in \mathcal{M} \text { such that } \mathcal{N}_{K} \not \subset G_{K}, \quad\left(\sum_{L \in \mathcal{N}_{K} \backslash G_{K}} a_{L} \boldsymbol{n}_{K \mid L}=0\right) \Rightarrow\left(\forall L \in \mathcal{N}_{K} \backslash G_{K}, a_{L}=0\right)
$$

In Figure 2 below, we show examples of clusters which satisfy (top line of the picture) this property (for triangles and rectangles), and which do not satisfy this property (bottom line). Roughly speaking, assumption (3.1) implies that there should not be too many edges of a control volume which are external edges of the cluster to which this control volume belongs, since, in dimension $d, d+1$ vectors are always linearly dependent. We will prove hereafter that this very simple geometrical relation is equivalent to the inf-sup stability of the pair of approximation spaces composed of the classical cell-centered finite volume approximation for the velocity 
and an approximation of the pressure which is piecewise constant over each cluster. We then set:

$$
\begin{aligned}
& \operatorname{regul}(\mathcal{G})=\inf \left\{I_{K}, K \in \mathcal{M}, \mathcal{N}_{K} \not \subset G_{K}\right\} \cup\left\{\frac{1}{\operatorname{card}(G)}, G \in \mathcal{G}\right\} \quad \text { with } \\
& I_{K}=\inf \left\{\left|\sum_{L \in \mathcal{N}_{K} \backslash G_{K}} a_{L} \boldsymbol{n}_{K \mid L}\right|^{2},\left(a_{L}\right)_{L \in \mathcal{N}_{K} \backslash G_{K}} \subset \mathbb{R} \text { and } \sum_{L \in \mathcal{N}_{K} \backslash G_{K}} a_{L}^{2}=1\right\} .
\end{aligned}
$$

We also define the function $\operatorname{regul}(\mathcal{D})$ by:

$$
\begin{gathered}
\operatorname{regul}(\mathcal{D})=\inf \left\{\frac{m_{\sigma}}{h_{K}^{d-1}}, \frac{d_{K, \sigma}}{h_{K}}, K \in \mathcal{M}, \sigma \in \mathcal{E}(K)\right\} \cup\left\{\frac{d_{K, K \mid L}}{d_{K \mid L}}, K \in \mathcal{M}, L \in \mathcal{N}_{K}\right\} \\
\cup\left\{\frac{h_{K}}{h_{L}}, K \in \mathcal{M}, L \in \mathcal{N}_{K}\right\} \cup\left\{\frac{1}{\operatorname{card}(\mathcal{E}(K))}, K \in \mathcal{M}\right\}
\end{gathered}
$$

and, finally, the regularity of the mesh is measured through the function:

$$
\operatorname{regul}(\mathcal{D}, \mathcal{G})=\inf \{\operatorname{regul}(\mathcal{D}), \operatorname{regul}(\mathcal{G})\}
$$

Super-admissible discretizations $\mathcal{D}$ such that $\operatorname{regul}(\mathcal{D}, \mathcal{G})>0$ are easily encountered: for example, one can consider in 2D a rectangular mesh, each cluster being defined by the rectangles sharing a same vertex. Another example is a mesh of triangles with all angles acute, in order that the circumcenter of each triangle be located inside the triangle, and the clusters are pairs of triangles. Both examples are depicted in the first line of Figure 2. In 3D, the assumption of "super-admissibility" is unfortunately only known to be satisfied by rectangular grids. However, the scheme is shown to be stable without this assumption; indeed the super-admissibility is only required in our convergence proof in order to obtain enough consistency on the divergence operator (estimate (4.23) of Thm. 4.7). Note also that adaptations of the scheme presented in this paper to circumvent this limitation are under study [10].

\subsection{Approximation spaces}

\subsubsection{The space $\mathrm{H}_{\mathcal{D}}(\Omega)$}

Let $\Omega$ be an open bounded polygonal $(d=2)$ or polyhedral $(d=3)$ subset of $\mathbb{R}^{d}$. Let $\mathcal{D}=(\mathcal{M}, \mathcal{E}, \mathcal{P})$ be an admissible finite volume discretization of $\Omega$ in the sense of Definition 3.1. We denote by $\mathrm{H}_{\mathcal{D}}(\Omega) \subset \mathrm{L}^{2}(\Omega)$ the space of functions which are piecewise constant on each control volume $K \in \mathcal{M}$. For all $v \in \mathrm{H}_{\mathcal{D}}(\Omega)$ and for all $K \in \mathcal{M}$, we denote by $v_{K}$ the constant value of $v$ in $K$.

\subsubsection{Bilinear forms, norms and semi-norms associated to $\mathrm{H}_{\mathcal{D}}(\Omega)$}

For $(v, w) \in\left(\mathrm{H}_{\mathcal{D}}(\Omega)\right)^{2}$, we define the following inner product, which is the discrete analogue of the canonical $\mathrm{H}_{0}^{1}(\Omega)$ bilinear form:

$$
[v, w]_{\mathcal{D}}=\sum_{\sigma \in \mathcal{E}_{\text {int }}, \sigma=K \mid L} \frac{m_{\sigma}}{d_{\sigma}}\left(v_{L}-v_{K}\right)\left(w_{L}-w_{K}\right)+\sum_{\sigma \in \mathcal{E}_{\text {ext }}, \sigma \in \mathcal{E}(K)} \frac{m_{\sigma}}{d_{K, \sigma}} v_{K} w_{K}
$$

We then obtain a norm in $\mathrm{H}_{\mathcal{D}}(\Omega)$ (thanks to the discrete Poincaré inequality (3.6) given below) by:

$$
\|w\|_{\mathcal{D}}=\left([w, w]_{\mathcal{D}}\right)^{1 / 2} .
$$


These definitions naturally extend to vector valued functions as follows. For $\boldsymbol{v}=\left(\boldsymbol{v}^{(i)}\right)_{i=1, \ldots, d} \in \mathrm{H}_{\mathcal{D}}(\Omega)^{d}$ and $\boldsymbol{w}=\left(\boldsymbol{w}^{(i)}\right)_{i=1, \ldots, d} \in \mathrm{H}_{\mathcal{D}}(\Omega)^{d}$, we define:

$$
\|\boldsymbol{v}\|_{\mathcal{D}}=\left(\sum_{i=1}^{d}\left[\boldsymbol{v}^{(i)}, \boldsymbol{v}^{(i)}\right]_{\mathcal{D}}\right)^{1 / 2}, \quad[\boldsymbol{v}, \boldsymbol{w}]_{\mathcal{D}}=\sum_{i=1}^{d}\left[\boldsymbol{v}^{(i)}, \boldsymbol{w}^{(i)}\right]_{\mathcal{D}}
$$

The discrete Poincaré inequality (see [11]) reads:

$$
\|w\|_{\mathrm{L}^{2}(\Omega)} \leq \operatorname{diam}(\Omega)\|w\|_{\mathcal{D}}, \forall w \in \mathrm{H}_{\mathcal{D}}(\Omega) .
$$

We define a discrete $\mathrm{H}^{-1}(\Omega)^{d}$ norm, which reads, for any function $\boldsymbol{f}$ of $\mathrm{L}^{2}(\Omega)^{d}$ :

$$
\|\boldsymbol{f}\|_{-1, \mathcal{D}}=\sup _{\boldsymbol{v} \in \mathrm{H}_{\mathcal{D}}(\Omega)^{d}} \frac{\int_{\Omega} \boldsymbol{f}(\boldsymbol{x}) \cdot \boldsymbol{v}(\boldsymbol{x}) \mathrm{d} \boldsymbol{x}}{\|\boldsymbol{v}\|_{\mathcal{D}}} .
$$

By the discrete Poincaré inequality, we obtain that this norm is controlled by the $\mathrm{L}^{2}$ norm:

$$
\|\boldsymbol{f}\|_{-1, \mathcal{D}} \leq \operatorname{diam}(\Omega)\|\boldsymbol{f}\|_{\mathrm{L}^{2}(\Omega)^{d}} .
$$

Finally, we define the three following bilinear forms over $\mathrm{H}_{\mathcal{D}}(\Omega) \times \mathrm{H}_{\mathcal{D}}(\Omega)$ by the following relations, which hold for any function $v$ and $w$ of $\mathrm{H}_{\mathcal{D}}(\Omega)$ :

$$
\begin{aligned}
\langle v, w\rangle_{\mathcal{M}} & =\frac{1}{2} \sum_{K \in \mathcal{M}} \sum_{L \in \mathcal{N}_{K}} m_{K \mid L}\left(h_{K}+h_{L}\right)\left(v_{L}-v_{K}\right)\left(w_{L}-w_{K}\right) \\
\langle v, w\rangle_{\mathcal{G}} & =\frac{1}{2} \sum_{K \in \mathcal{M}} \sum_{L \in \mathcal{N}_{K} \cap G_{K}} m_{K \mid L}\left(h_{K}+h_{L}\right)\left(v_{L}-v_{K}\right)\left(w_{L}-w_{K}\right) \\
\langle v, w\rangle_{\mathcal{M} \backslash \mathcal{G}} & =\frac{1}{2} \sum_{K \in \mathcal{M}} \sum_{L \in \mathcal{N}_{K} \backslash G_{K}} m_{K \mid L}\left(h_{K}+h_{L}\right)\left(v_{L}-v_{K}\right)\left(w_{L}-w_{K}\right) .
\end{aligned}
$$

Note that $\langle v, w\rangle_{\mathcal{M}}=\langle v, w\rangle_{\mathcal{G}}+\langle v, w\rangle_{\mathcal{M} \backslash \mathcal{G}}$. Each of these three bilinear forms defines a semi-norm over $\mathrm{H}_{\mathcal{D}}(\Omega)$ :

$$
|w|_{\mathcal{M}}=\langle w, w\rangle_{\mathcal{M}}^{1 / 2} \quad|w|_{\mathcal{G}}=\langle w, w\rangle_{\mathcal{G}}^{1 / 2} \quad|w|_{\mathcal{M} \backslash \mathcal{G}}=\langle w, w\rangle_{\mathcal{M} \backslash \mathcal{G}}^{1 / 2}
$$

\subsubsection{Interpolation operators}

We define the interpolation operator $P_{\mathcal{D}}$, mapping $C(\Omega)$ onto $\mathrm{H}_{\mathcal{D}}(\Omega)$, by setting $\left(P_{\mathcal{D}} \varphi\right)_{K}=\varphi\left(\boldsymbol{x}_{K}\right)$, for all $K \in \mathcal{M}$, for all $\varphi \in C(\Omega)$. Its natural extension to vector valued functions, also noted $P_{\mathcal{D}}$, maps $C(\Omega)^{d}$ onto $\mathrm{H}_{\mathcal{D}}(\Omega)^{d}$, by $\left(P_{\mathcal{D}} \boldsymbol{\varphi}\right)_{K}=\boldsymbol{\varphi}\left(\boldsymbol{x}_{K}\right)$, for all $K \in \mathcal{M}$, for all $\boldsymbol{\varphi} \in C(\Omega)^{d}$.

We also define two additional interpolation operators, $P_{\mathcal{M}}$ and $P_{\mathcal{G}}$. The first one maps $\mathrm{L}^{2}(\Omega)$ onto $\mathrm{H}_{\mathcal{D}}(\Omega)$, the second one maps $\mathrm{L}^{2}(\Omega)$ onto the sub-space of functions of $\mathrm{H}_{\mathcal{D}}(\Omega)$ which are constant over each cluster:

$$
\left(P_{\mathcal{M}} \varphi\right)_{K}=\frac{1}{m_{K}} \int_{K} \varphi(\boldsymbol{x}) \mathrm{d} \boldsymbol{x} \quad\left(P_{\mathcal{G}} \varphi\right)_{K}=\frac{1}{\sum_{L \in G_{K}} m_{L}} \int_{\cup_{L \in G_{K}}} \varphi(\boldsymbol{x}) \mathrm{d} \boldsymbol{x} .
$$

The operator $P_{\mathcal{M}}$ satisfies the following continuity result, the proof of which easily follows from estimates (A.8) and (A.9) (see Appendix, Lem. A.3). 
Lemma 3.3. Let assumption (2.2) hold, let $\mathcal{D}$ be an admissible discretization of $\Omega$ in the sense of Definition 3.1 and $\theta>0$ be such that $\operatorname{regul}(\mathcal{D})>\theta$. Let $\boldsymbol{v} \in \mathrm{H}_{0}^{1}(\Omega)^{d}$. Then the following bound holds:

$$
\left\|P_{\mathcal{M}}(\boldsymbol{v})\right\|_{\mathcal{D}} \leq c|\boldsymbol{v}|_{\mathrm{H}^{1}(\Omega)^{d}}
$$

where $c$ only depends on $\Omega$ and $\theta$ and $|\cdot|_{\mathrm{H}^{1}(\Omega)^{d}}$ stands for the $\mathrm{H}^{1}$ seminorm on $\Omega$.

\section{Approximation of the generalized Stokes problem}

To perform the convergence and error analysis for the generalised Stokes problem, we implement in this paper a step-by-step process, which has been described for a standard elliptic problem in [14]. The main advantage of this approach is that consistency residual estimates can be recast under a generic form, suitable for reutilization in subsequent works. These residuals are specific to the considered regularity for the continuous function, the projection operator onto the discrete space and the equation. With respect to this latter point, they may be decomposed as the sum of elementary consistency residuals, each one corresponding to a term of the equation: for the generalized Stokes problem addressed in this study, the zero order, diffusion, gradient, divergence and stabilization terms. We thus provide here an estimate for each of them, for the above defined (usual in the finite volume context) projection operators. Note that, by this last point, the results of this paper differ from those presented in [13], where the interpolation operators were built by a quasi-interpolation technique à la Clément (more classical in the finite element context).

The present section is built as follows. The finite volume is first defined (Sect. 4.1), then its stability is proven (Sect. 4.2). Residual estimates are given in Section 4.3; they are based on consistency results for local quantities (in particular, the finite volume fluxes) which are provided in the Appendix. These bounds allow to prove the convergence of the scheme (Sect. 4.4), and then to obtain, for regular solutions, error estimates (Sect. 4.5).

\subsection{The finite volume scheme}

Finite volume schemes are classically presented as discrete balance equations with a suitable approximation of the fluxes, see e.g. [11]. However, in recent works dealing with cell centered finite volume methods for elliptic problems [12,14], an equivalent variational formulation in adequate functional spaces is introduced, and this presentation is convenient for the analysis of the schemes, as it is a natural starting point to derive stability estimates. We follow here this latter path.

We begin by defining a discrete divergence operator $\operatorname{div}_{\mathcal{D}}$, the expression of which is the same as in [13], and which maps $\mathrm{H}_{\mathcal{D}}(\Omega)^{d}$ to $\mathrm{H}_{\mathcal{D}}(\Omega)$ and reads:

$$
\forall \boldsymbol{v} \in \mathrm{H}_{\mathcal{D}}(\Omega)^{d}, \quad \operatorname{div}_{\mathcal{D}} \boldsymbol{v}(\boldsymbol{x})=\left(\operatorname{div}_{\mathcal{D}} \boldsymbol{v}\right)_{K}=\frac{1}{m_{K}} \sum_{L \in \mathcal{N}_{K}} m_{K \mid L} \boldsymbol{n}_{K \mid L} \cdot \frac{d_{L, \sigma} \boldsymbol{v}_{K}+d_{K, \sigma} \boldsymbol{v}_{L}}{d_{K \mid L}}, \quad \forall \boldsymbol{x} \in K, \forall K \in \mathcal{M}
$$

The adjoint of this discrete divergence with respect to the discrete $\mathrm{L}^{2}$ inner product defines a discrete gradient $\boldsymbol{\nabla}_{\mathcal{D}}$, mapping $\mathrm{H}_{\mathcal{D}}(\Omega)$ to $\mathrm{H}_{\mathcal{D}}(\Omega)^{d}$, which is expressed as:

$$
\forall q \in \mathrm{H}_{\mathcal{D}}(\Omega), \quad \nabla_{\mathcal{D}} q(\boldsymbol{x})=\left(\boldsymbol{\nabla}_{\mathcal{D}} q\right)_{K}=\frac{1}{m_{K}} \sum_{L \in \mathcal{N}_{K}} m_{K \mid L} \frac{d_{L, \sigma}}{d_{K \mid L}}\left(q_{L}-q_{K}\right) \boldsymbol{n}_{K \mid L}, \quad \forall \boldsymbol{x} \in K, \forall K \in \mathcal{M}
$$


Since $\sum_{\sigma \in \mathcal{E}(K)} m_{\sigma} \boldsymbol{n}_{\sigma}=0$, this discrete gradient equivalently reads:

$$
\begin{aligned}
&\left(\boldsymbol{\nabla}_{\mathcal{D}} q\right)_{K}= \frac{1}{m_{K}}\left[\sum_{L \in \mathcal{N}_{K}} \boldsymbol{F}_{\mathrm{grad}, K \mid L}+\sum_{\sigma \in \mathcal{E}(K) \cap \mathcal{E}_{\mathrm{ext}}} \boldsymbol{F}_{\mathrm{grad}, \sigma}\right] \quad \text { with } \\
& \boldsymbol{F}_{\mathrm{grad}, K \mid L}=m_{K \mid L} \frac{d_{K, K \mid L} q_{K}+d_{L, K \mid L} q_{L}}{d_{K \mid L}} \boldsymbol{n}_{K \mid L}, \quad \boldsymbol{F}_{\mathrm{grad}, \sigma}=m_{\sigma} q_{K} \boldsymbol{n}_{\sigma}
\end{aligned}
$$

in which we recognize a classical "flux-based" finite volume formulation, with, however, a rather unnatural (and only first order consistent) interpolation of the pressure on the edge.

The discrete solution is then defined as the pair of functions $(\boldsymbol{u}, p)$ such that:

$$
\begin{array}{|ll}
\boldsymbol{u} \in \mathrm{H}_{\mathcal{D}}(\Omega)^{d}, p \in \mathrm{H}_{\mathcal{D}}(\Omega) \text { with } \int_{\Omega} p(\boldsymbol{x}) \mathrm{d} \boldsymbol{x}=0 \text { and: } & \\
\eta \int_{\Omega} \boldsymbol{u}(\boldsymbol{x}) \cdot \boldsymbol{v}(\boldsymbol{x}) \mathrm{d} \boldsymbol{x}+\nu[\boldsymbol{u}, \boldsymbol{v}]_{\mathcal{D}}-\int_{\Omega} p(\boldsymbol{x}) \operatorname{div}_{\mathcal{D}} \boldsymbol{v}(\boldsymbol{x}) \mathrm{d} \boldsymbol{x}=\int_{\Omega} \boldsymbol{f}(\boldsymbol{x}) \cdot \boldsymbol{v}(\boldsymbol{x}) \mathrm{d} \boldsymbol{x} & \forall \boldsymbol{v} \in \mathrm{H}_{\mathcal{D}}(\Omega)^{d} \\
\int_{\Omega} \operatorname{div}_{\mathcal{D}} \boldsymbol{u}(\boldsymbol{x}) q(\boldsymbol{x}) \mathrm{d} \boldsymbol{x}+\lambda\langle p, q\rangle_{\mathcal{G}}=\int_{\Omega} g(\boldsymbol{x}) q(\boldsymbol{x}) \mathrm{d} \boldsymbol{x} & \forall q \in \mathrm{H}_{\mathcal{D}}(\Omega)
\end{array}
$$

where $\lambda$ is a positive parameter. The term $\lambda\langle\cdot, \cdot\rangle_{\mathcal{G}}$ corresponds to a "cluster-wide" stabilization, in the sense that it involves pressure jumps only across the internal edges of the clusters. Thanks to the definition of the operator $\operatorname{div}_{\mathcal{D}}$ and of the bilinear form $\langle\cdot, \cdot\rangle_{\mathcal{G}}$, and thanks to the assumption that the mean value of $g$ is zero, we observe that both sides of the second equation vanish for any constant test function $q$; in spite of the assumption $\int_{\Omega} p(\boldsymbol{x}) \mathrm{d} \boldsymbol{x}=0$, system (4.4) is thus equivalent to a square linear system.

System (4.4) is equivalent to searching for the family of vectors $\left(\boldsymbol{u}_{K}\right)_{K \in \mathcal{M}}$ of $\mathbb{R}^{d}$, and scalars $\left(p_{K}\right)_{K \in \mathcal{M}}$ solution to the system of equations (written under flux form) obtained by writing for each control volume $K$ of $\mathcal{M}$ :

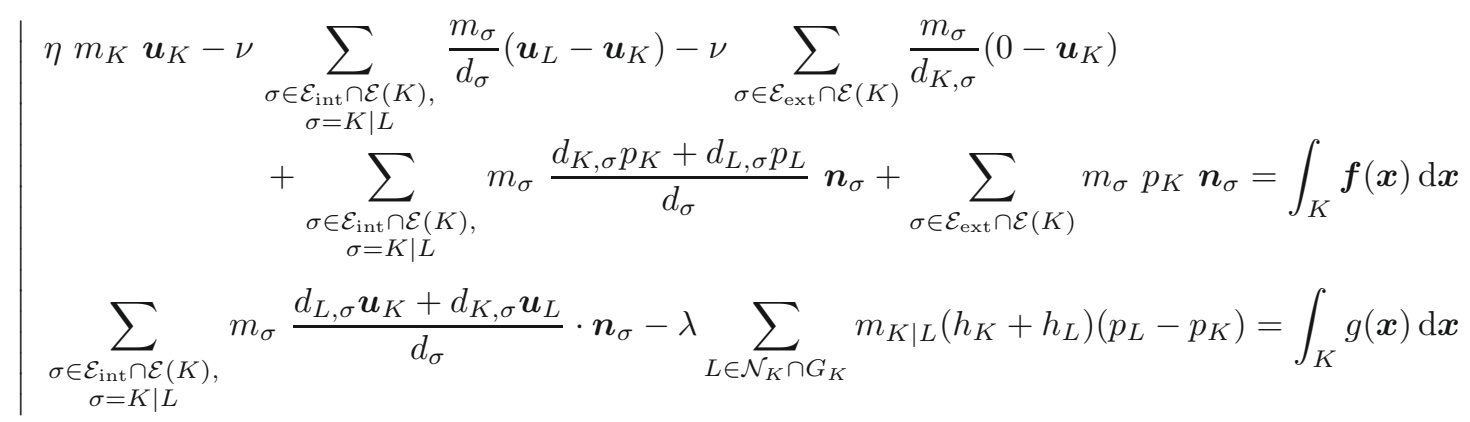

supplemented by the relation:

$$
\sum_{K \in \mathcal{M}} m_{K} p_{K}=0
$$

\subsection{Stability of the scheme}

This section is aimed at proving the stability of the scheme. It is worth stressing that, to this purpose, the mesh is not required to be "super-admissible", but only admissible in the sense of Definition 3.1. 
We first begin by a (partial) stability result for the discrete gradient operator, which is not specific to a clustered approximation, and may be seen as the "finite volume analogue" to a lemma already known in the finite element context [17], Lemma 3.3.

Lemma 4.1. Let $\mathcal{D}$ be an admissible discretization of $\Omega$ in the sense of Definition 3.1. Let $\theta>0$ be such that $\operatorname{regul}(\mathcal{D})>\theta$. Then there exists two strictly positive real numbers $\beta_{1}$ and $\beta_{2}$ depending only on $d, \Omega$ and $\theta$ such that the following property holds:

$$
\begin{aligned}
& \forall p \in \mathrm{H}_{\mathcal{D}}(\Omega) \text { with } \int_{\Omega} p(\boldsymbol{x}) \mathrm{d} \boldsymbol{x}=0, \exists \boldsymbol{v} \in \mathrm{H}_{\mathcal{D}}(\Omega)^{d} \\
& \text { such that } \mid \begin{array}{ll}
\|\boldsymbol{v}\|_{\mathcal{D}} \leq \beta_{1}\|p\|_{\mathrm{L}^{2}(\Omega)} \quad \text { and } \\
\int_{\Omega} p(\boldsymbol{x}) \operatorname{div}_{\mathcal{D}} \boldsymbol{v}(\boldsymbol{x}) \mathrm{d} \boldsymbol{x} \geq\|p\|_{\mathrm{L}^{2}(\Omega)}^{2}-\beta_{2}|p|_{\mathcal{M}}^{2} .
\end{array}
\end{aligned}
$$

Proof. Let $p \in \mathrm{H}_{\mathcal{D}}(\Omega)$ be given. We apply a classical result (e.g. [19], Cor. 2.1), the proof of which relies on arguments which may be traced back to Nečas [20]. Because $p(\boldsymbol{x})$ is a zero mean-valued function, there exists $c_{\mathrm{dr}}$, which only depends on $d$ and $\Omega$, and $\overline{\boldsymbol{v}} \in \mathrm{H}_{0}^{1}(\Omega)^{d}$ such that $\operatorname{div} \overline{\boldsymbol{v}}(\boldsymbol{x})=p(\boldsymbol{x})$ for a.e. $\boldsymbol{x} \in \Omega$ and:

$$
\|\overline{\boldsymbol{v}}\|_{\mathrm{H}^{1}(\Omega)^{d}} \leq c_{\mathrm{dr}}\|p\|_{\mathrm{L}^{2}(\Omega)} .
$$

We then set:

$$
\boldsymbol{v}_{\sigma}^{(i)}=\frac{1}{m_{\sigma}} \int_{\sigma} \overline{\boldsymbol{v}}^{(i)}(\boldsymbol{x}) \mathrm{d} \gamma(\boldsymbol{x}), \quad \forall \sigma \in \mathcal{E}, i=1, \ldots, d
$$

(note that $\boldsymbol{v}_{\sigma}^{(i)}=0$ for all $\sigma \in \mathcal{E}_{\text {ext }}$ and $i=1, \ldots, d$ ) and we define $\boldsymbol{v} \in \mathrm{H}_{\mathcal{D}}(\Omega)^{d}$ by:

$$
\boldsymbol{v}_{K}^{(i)}=\frac{1}{m_{K}} \int_{K} \overline{\boldsymbol{v}}^{(i)}(\boldsymbol{x}) \mathrm{d} \boldsymbol{x}, \quad \forall K \in \mathcal{M}, i=1, \ldots, d .
$$

As proved in the Appendix (relation (A.8)), we know that there exists $c_{1}$ only depending on $d, \Omega$ and $\theta$ such that:

$$
\forall K \in \mathcal{M}, \forall \sigma \in \mathcal{E}(K), \quad\left|\boldsymbol{v}_{K}-\boldsymbol{v}_{\sigma}\right|^{2} \leq c_{1} \frac{h_{K}}{m_{\sigma}} \int_{K}|\nabla \overline{\boldsymbol{v}}(\boldsymbol{x})|^{2} \mathrm{~d} \boldsymbol{x} .
$$

In addition, by the continuity of the interpolation operator $P_{\mathcal{M}}$ (Lem. 3.3), there exists another real number $c_{2}$ once again only depending on $d, \Omega$ and $\theta$ such that:

$$
\|\boldsymbol{v}\|_{\mathcal{D}} \leq c_{2}\|\overline{\boldsymbol{v}}\|_{\mathrm{H}^{1}(\Omega)^{d}} \leq c_{2} c_{\mathrm{dr}}\|p\|_{\mathrm{L}^{2}(\Omega)} .
$$

We then have:

$$
\int_{\Omega} p(\boldsymbol{x}) \operatorname{div}_{\mathcal{D}} \boldsymbol{v}(\boldsymbol{x}) \mathrm{d} \boldsymbol{x}=\sum_{K \in \mathcal{M}} p_{K} \sum_{\substack{\sigma \in \mathcal{E}_{\mathrm{int}} \cap \mathcal{E}(K), \sigma=K \mid L}} m_{\sigma} \boldsymbol{n}_{\sigma} \cdot \frac{d_{L, \sigma} \boldsymbol{v}_{K}+d_{K, \sigma} \boldsymbol{v}_{L}}{d_{\sigma}}=T_{1}+T_{2}
$$

where:

$$
\begin{aligned}
T_{1}=\sum_{K \in \mathcal{M}} p_{K} \sum_{\substack{\sigma \in \mathcal{E}_{\operatorname{int}} \cap \mathcal{E}(K), \sigma=K \mid L}} m_{\sigma} \boldsymbol{n}_{\sigma} \cdot \boldsymbol{v}_{\sigma} & =\sum_{K \in \mathcal{M}} p_{K} \sum_{\substack{\sigma \in \mathcal{E}_{\operatorname{int}} \cap \mathcal{E}(K), \sigma=K \mid L}} \int_{\sigma} \overline{\boldsymbol{v}}(\boldsymbol{x}) \cdot \boldsymbol{n}_{\sigma} \mathrm{d} \gamma(\boldsymbol{x}) \\
& =\int_{\Omega} p(\boldsymbol{x}) \operatorname{div} \overline{\boldsymbol{v}}(\boldsymbol{x}) \mathrm{d} \boldsymbol{x}=\|p\|_{\mathrm{L}^{2}(\Omega)}^{2}
\end{aligned}
$$


and:

$$
\begin{aligned}
T_{2} & =\sum_{K \in \mathcal{M}} p_{K} \sum_{\substack{\sigma \in \mathcal{E}_{\mathrm{int}} \cap \mathcal{E}(K), \sigma=K \mid L}} m_{\sigma}\left(\frac{d_{L, \sigma} \boldsymbol{v}_{K}+d_{K, \sigma} \boldsymbol{v}_{L}}{d_{\sigma}}-\boldsymbol{v}_{\sigma}\right) \cdot \boldsymbol{n}_{\sigma} \\
& =\sum_{\sigma \in \mathcal{E}_{\text {int }}, \sigma=K \mid L} m_{\sigma}\left(p_{K}-p_{L}\right)\left(\frac{d_{L, \sigma} \boldsymbol{v}_{K}+d_{K, \sigma} \boldsymbol{v}_{L}}{d_{\sigma}}-\boldsymbol{v}_{\sigma}\right) \cdot \boldsymbol{n}_{\sigma} .
\end{aligned}
$$

We then have, thanks to the Cauchy-Schwarz inequality:

$$
T_{2}^{2} \leq\left[\sum_{\sigma \in \mathcal{E}_{\mathrm{int}}, \sigma=K \mid L} m_{\sigma}\left(h_{K}+h_{L}\right)\left(p_{K}-p_{L}\right)^{2}\right]\left[\sum_{\sigma \in \mathcal{E}_{\mathrm{int}}, \sigma=K \mid L} \frac{m_{\sigma}}{h_{K}+h_{L}}\left|\frac{d_{L, \sigma} \boldsymbol{v}_{K}+d_{K, \sigma} \boldsymbol{v}_{L}}{d_{\sigma}}-\boldsymbol{v}_{\sigma}\right|^{2}\right]
$$

We now remark that, since, for any internal edge $\sigma=K \mid L, d_{K, \sigma}<d_{\sigma}, d_{L, \sigma}<d_{\sigma}$ and $d_{\sigma}=d_{K, \sigma}+d_{L, \sigma}$ :

$$
\left|\frac{d_{L, \sigma} \boldsymbol{v}_{K}+d_{K, \sigma} \boldsymbol{v}_{L}}{d_{\sigma}}-\boldsymbol{v}_{\sigma}\right|^{2} \leq 2\left|\boldsymbol{v}_{K}-\boldsymbol{v}_{\sigma}\right|^{2}+2\left|\boldsymbol{v}_{L}-\boldsymbol{v}_{\sigma}\right|^{2} .
$$

Using inequalities (4.9) and (4.8) and noting that, thanks to the assumption $\operatorname{regul}(\mathcal{D})>\theta$, the maximum number of edges for a control volume is bounded, we get:

$$
T_{2} \leq c_{3}|p|_{\mathcal{M}}\|\overline{\boldsymbol{v}}\|_{\mathrm{H}^{1}(\Omega)^{d}} \leq c_{3} c_{\mathrm{dr}}|p|_{\mathcal{M}}\|p\|_{\mathrm{L}^{2}(\Omega)}
$$

where $c_{3}$ only depends on $d, \Omega$ and $\theta$. Collecting terms, we obtain by Young's inequality:

$$
\int_{\Omega} p(\boldsymbol{x}) \operatorname{div}_{\mathcal{D}} \boldsymbol{v}(\boldsymbol{x}) \mathrm{d} \boldsymbol{x} \geq \frac{1}{2}\|p\|_{\mathrm{L}^{2}(\Omega)}^{2}-\frac{\left(c_{3} c_{\mathrm{dr}}\right)^{2}}{2}|p|_{\mathcal{M}}^{2}
$$

which, together with relation (4.10), concludes the proof.

The following result is an essential stability feature of the scheme. It proves, in particular, that the simple geometrical condition $\operatorname{regul}(\mathcal{G})>\theta>0$, where $\operatorname{regul}(\mathcal{G})$ is given by relation $(3.2)$, yields the inf-sup stability of the discretization with cluster-wide constant pressures (see remark below), provided that the mesh is regular.

Lemma 4.2. Under hypotheses (2.2)-(2.4), let $\mathcal{D}$ be an admissible discretization of $\Omega$ in the sense of Definition 3.1. Let $\theta>0$ be such that $\operatorname{regul}(\mathcal{D}, \mathcal{G})>\theta$. Then there exist two positive real numbers, again denoted by $\beta_{1}$ and $\beta_{2}$, depending only on $d, \Omega$ and $\theta$, such that the following property holds:

$$
\begin{aligned}
& \forall p \in \mathrm{H}_{\mathcal{D}}(\Omega) \text { with } \int_{\Omega} p(\boldsymbol{x}) \mathrm{d} \boldsymbol{x}=0, \exists \boldsymbol{v} \in \mathrm{H}_{\mathcal{D}}(\Omega)^{d} \\
& \qquad \begin{array}{l|l}
\|\boldsymbol{v}\|_{\mathcal{D}} \leq \beta_{1}\|p\|_{\mathrm{L}^{2}(\Omega)} \quad \text { and that } & \begin{array}{l}
\int_{\Omega} p(\boldsymbol{x}) \operatorname{div}_{\mathcal{D}} \boldsymbol{v}(\boldsymbol{x}) \mathrm{d} \boldsymbol{x} \geq\|p\|_{\mathrm{L}^{2}(\Omega)}^{2}-\beta_{2}|p|_{\mathcal{G}}^{2}
\end{array}
\end{array}
\end{aligned}
$$

Proof. Let $p \in \mathrm{H}_{\mathcal{D}}(\Omega)$ be given. We define $\boldsymbol{v} \in \mathrm{H}_{\mathcal{D}}(\Omega)^{d}$ by:

$$
\boldsymbol{v}_{K}=\frac{1}{h_{K}^{d-2}} \sum_{L \in \mathcal{N}_{K} \backslash G_{K}} m_{K \mid L} \frac{d_{L, K \mid L}}{d_{K \mid L}}\left(p_{L}-p_{K}\right) \boldsymbol{n}_{K \mid L} .
$$


As the discrete divergence is the transpose of the discrete gradient, we have:

$$
\begin{aligned}
\int_{\Omega} p(\boldsymbol{x}) \operatorname{div}_{\mathcal{D}}(-\boldsymbol{v})(\boldsymbol{x}) \mathrm{d} \boldsymbol{x}=\int_{\Omega} \boldsymbol{\nabla}_{\mathcal{D}} p(\boldsymbol{x}) \cdot \boldsymbol{v}(\boldsymbol{x}) \mathrm{d} \boldsymbol{x} \\
=\sum_{K \in \mathcal{M}} \boldsymbol{v}_{K} \cdot[\underbrace{\sum_{L \in \mathcal{N}_{K} \backslash G_{K}} m_{K \mid L} \frac{d_{L, K \mid L}}{d_{K \mid L}}\left(p_{L}-p_{K}\right) \boldsymbol{n}_{K \mid L}}_{\boldsymbol{T}_{1, K}}+\underbrace{\sum_{L \in \mathcal{N}_{K} \cap G_{K}} m_{K \mid L} \frac{d_{L, K \mid L}}{d_{K \mid L}}\left(p_{L}-p_{K}\right) \boldsymbol{n}_{K \mid L}}_{\boldsymbol{T}_{2, K}}]
\end{aligned}
$$

Remarking that $\boldsymbol{v}_{K}=\frac{1}{h_{K}^{d-2}} \boldsymbol{T}_{1, K}$, we have by Young's inequality:

$$
\int_{\Omega} p(\boldsymbol{x}) \operatorname{div}_{\mathcal{D}}(-\boldsymbol{v})(\boldsymbol{x}) \mathrm{d} \boldsymbol{x} \geq \frac{1}{2} \sum_{K \in \mathcal{M}} \frac{1}{h_{K}^{d-2}}\left(\left|\boldsymbol{T}_{1, K}\right|^{2}-\left|\boldsymbol{T}_{2, K}\right|^{2}\right) .
$$

Thanks to the regularity assumption on the mesh (in particular the fact that the quantity $\operatorname{regul}(\mathcal{G})$, defined by relation (3.2), is greater than $\theta$ ), the first part of this summation satisfies the following estimate:

$$
\begin{aligned}
\frac{1}{2} \sum_{K \in \mathcal{M}} \frac{1}{h_{K}^{d-2}}\left|\boldsymbol{T}_{1, K}\right|^{2} & =\frac{1}{2} \sum_{K \in \mathcal{M}} \frac{1}{h_{K}^{d-2}}\left|\sum_{L \in \mathcal{N}_{K} \backslash G_{K}} m_{K \mid L} \frac{d_{L, K \mid L}}{d_{K \mid L}}\left(p_{L}-p_{K}\right) \boldsymbol{n}_{K \mid L}\right|^{2} \\
& \geq \frac{\theta}{2} \sum_{K \in \mathcal{M}} \frac{1}{h_{K}^{d-2}} \sum_{L \in \mathcal{N}_{K} \backslash G_{K}} m_{K \mid L}^{2}\left(\frac{d_{L, K \mid L}}{d_{K \mid L}}\right)^{2}\left(p_{L}-p_{K}\right)^{2} \\
& =\frac{\theta}{2} \sum_{K \in \mathcal{M}} \sum_{L \in \mathcal{N}_{K} \backslash G_{K}} \frac{m_{K \mid L}}{h_{K}^{d-2}\left(h_{K}+h_{L}\right)}\left(\frac{d_{L, K \mid L}}{d_{K \mid L}}\right)^{2} m_{K \mid L}\left(h_{K}+h_{L}\right)\left(p_{L}-p_{K}\right)^{2}
\end{aligned}
$$

and thus, by regularity assumptions on the mesh, there exists $c_{1}>0$ depending only on $\theta$ such that:

$$
\frac{1}{2} \sum_{K \in \mathcal{M}} \frac{1}{h_{K}^{d-2}}\left|\boldsymbol{T}_{1, K}\right|^{2} \geq c_{1}|p|_{\mathcal{M} \backslash \mathcal{G}}^{2}
$$

By a similar computation, we get:

$$
\begin{aligned}
\frac{1}{2} \sum_{K \in \mathcal{M}} \frac{1}{h_{K}^{d-2}}\left|\boldsymbol{T}_{2, K}\right|^{2} & \leq \frac{1}{2} \sum_{K \in \mathcal{M}} \sum_{L \in \mathcal{N}_{K} \cap G_{K}} \frac{m_{K \mid L}}{h_{K}^{d-2}\left(h_{K}+h_{L}\right)}\left(\frac{d_{L, K \mid L}}{d_{K \mid L}}\right)^{2} m_{K \mid L}\left(h_{K}+h_{L}\right)\left(p_{L}-p_{K}\right)^{2} \\
& \leq c_{2}|p|_{\mathcal{G}}^{2}
\end{aligned}
$$

where, once again, $c_{2}$ only depends on the regularity of the mesh. Thus, collecting the bounds, we get:

$$
\int_{\Omega} p(\boldsymbol{x}) \operatorname{div}_{\mathcal{D}}(-\boldsymbol{v})(\boldsymbol{x}) \mathrm{d} \boldsymbol{x} \geq c_{1}|p|_{\mathcal{M} \backslash \mathcal{G}}^{2}-c_{2}|p|_{\mathcal{G}}^{2}
$$


On the other hand, because, by assumption, the number of edges of the control volumes is bounded, we have:

$$
\begin{aligned}
\|\boldsymbol{v}\|_{\mathcal{D}}^{2} \leq & 2 \sum_{\sigma \in \mathcal{E}_{\text {int }}, \sigma=K \mid L} \frac{m_{\sigma}}{d_{\sigma}}\left(\left|\boldsymbol{v}_{K}\right|^{2}+\left|\boldsymbol{v}_{L}\right|^{2}\right)+\sum_{\sigma \in \mathcal{E}_{\text {ext }} \cap \mathcal{E}(K)} \frac{m_{\sigma}}{d_{\sigma}}\left|\boldsymbol{v}_{K}\right|^{2} \\
\leq & c \sum_{\sigma \in \mathcal{E}_{\text {int }}, \sigma=K \mid L} \frac{m_{\sigma}}{d_{\sigma}}\left[\sum_{M \in \mathcal{N}_{K}} \frac{\left(m_{K \mid M}\right)^{2}}{h_{K}^{2(d-2)}}\left(p_{K}^{2}+p_{M}^{2}\right)+\sum_{M \in \mathcal{N}_{L}} \frac{\left(m_{L \mid M}\right)^{2}}{h_{L}^{2(d-2)}}\left(p_{L}^{2}+p_{M}^{2}\right)\right] \\
& +c \sum_{\sigma \in \mathcal{E}_{\text {ext }} \cap \mathcal{E}(K)} \frac{m_{\sigma}}{d_{\sigma}} \sum_{M \in \mathcal{N}_{K}} \frac{\left(m_{K \mid M)^{2}}\right.}{h_{K}^{2(d-2)}}\left(p_{K}^{2}+p_{M}^{2}\right) .
\end{aligned}
$$

Reordering the summations, the above relation takes the following form:

$$
\|\boldsymbol{v}\|_{\mathcal{D}}^{2} \leq \sum_{K \in \mathcal{M}} \mu_{K} p_{K}^{2}
$$

and the regularity of the mesh implies that each coefficient $\mu_{K} \leq c m_{K}$ where $c$ only depends on $\theta$ and $d$; note that we use here the assumption that the ratio of the measure of two neighbouring volumes is bounded. We then get:

$$
\|\boldsymbol{v}\|_{\mathcal{D}}^{2} \leq c_{3}\|p\|_{\mathrm{L}^{2}(\Omega)}^{2} .
$$

Hence, estimates (4.12) and (4.13) prove the existence of $\boldsymbol{v}_{1} \in \mathrm{H}_{\mathcal{D}}(\Omega)^{d}$ such that:

$$
\int_{\Omega} p(\boldsymbol{x}) \operatorname{div}_{\mathcal{D}} \boldsymbol{v}_{1}(\boldsymbol{x}) \mathrm{d} \boldsymbol{x} \geq \beta_{2}|p|_{\mathcal{M} \backslash \mathcal{G}}^{2}-c_{4}|p|_{\mathcal{G}}^{2}, \quad\left\|\boldsymbol{v}_{1}\right\|_{\mathcal{D}}^{2} \leq c_{5}\|p\|_{\mathrm{L}^{2}(\Omega)}
$$

where the real numbers $c_{4}$ and $c_{5}$ only depend on the regularity of the mesh. Let $\boldsymbol{v}_{2} \in \mathrm{H}_{\mathcal{D}}(\Omega)^{d}$ be such that the estimate of Lemma 4.1 holds; then the desired result is obtained with $\boldsymbol{v}=\boldsymbol{v}_{1}+\boldsymbol{v}_{2}$.

Remark 4.3. We easily note that $|p|_{\mathcal{G}}$ vanishes for pressure fields which are constant over each cluster. The previous result thus shows that combining an approximation of the velocity by the space $\mathrm{H}_{\mathcal{D}}(\Omega)^{d}$ and an approximation of the pressure by the functions in $\mathrm{H}_{\mathcal{D}}(\Omega)$ which are constant on each cluster yields an inf-sup stable discretization, which should be quite usable in practice. However, letting the pressure vary within the clusters and adding a stabilization term is both easier to implement and, from numerical experiments, more accurate.

This result suggests that the scheme under consideration may be in some particular cases obtained via a minimal stabilization procedure as defined in [4]; an example of such a derivation is given in [16].

We are now in position to prove stability estimates for the velocity and the pressure. In the following result, we split the right-hand side $f$ in two parts and we control the unknowns by the $\mathrm{L}^{2}$ norm of the first one and the discrete $\mathrm{H}^{-1}$ norm of the second one; this decomposition is here rather artificial, but will be crucial further, when applying this stability result to derive error estimates.

Theorem 4.4 (estimates for the velocity and the pressure). We suppose that hypotheses (2.2)-(2.4) hold. Let $\mathcal{D}$ be an admissible discretization of $\Omega$ in the sense of Definition 3.1 and let $\theta>0$ be such that $\operatorname{regul}(\mathcal{D}, \mathcal{G})>\theta$. Let $\lambda \in(0,+\infty)$ be given. Let $(\boldsymbol{u}, p) \in \mathrm{H}_{\mathcal{D}}(\Omega)^{d} \times \mathrm{H}_{\mathcal{D}}(\Omega)$ be a solution to (4.4). Then, for any $\boldsymbol{f}_{1}$ and $\boldsymbol{f}_{2}$ in $\mathrm{L}^{2}(\Omega)^{d}$ such that $\boldsymbol{f}=\boldsymbol{f}_{1}+\boldsymbol{f}_{2}$, there exist two constants $c_{2}$ and $c_{3}$ together with two positive real numbers $c_{1}$ and $c_{4}$ depending only on $d, \Omega$ and $\theta$ such that the following inequality holds:

$\eta\|\boldsymbol{u}\|_{\mathrm{L}^{2}(\Omega)^{d}}^{2}+\nu\|\boldsymbol{u}\|_{\mathcal{D}}^{2}+\frac{c_{1}}{\max [\eta, \nu, 1 / \lambda]}\|p\|_{\mathrm{L}^{2}(\Omega)}^{2}+\lambda|p|_{\mathcal{G}}^{2} \leq \frac{c_{2}}{\eta}\left\|\boldsymbol{f}_{1}\right\|_{\mathrm{L}^{2}(\Omega)^{d}}^{2}+\frac{c_{3}}{\nu}\left\|\boldsymbol{f}_{2}\right\|_{-1, \mathcal{D}}^{2}+c_{4} \max [\eta, \nu, 1 / \lambda]\|g\|_{\mathrm{L}^{2}(\Omega)}^{2}$. 
Remark 4.5 (dependence of the stability estimate on the stabilization parameter). We note that the bound for the $L^{2}$ estimate for the pressure blows up when $\lambda$ tends to zero, as $|\cdot|_{\mathcal{G}}$ is a very weak seminorm which vanishes for any constant-by-cluster pressure field: the stabilization of the scheme is thus necessary to control the pressure.

Proof. Let $(\boldsymbol{u}, p) \in \mathrm{H}_{\mathcal{D}}(\Omega)^{d} \times \mathrm{H}_{\mathcal{D}}(\Omega)$ be a solution to (4.4). First, we chose $\boldsymbol{w} \in \mathrm{H}_{\mathcal{D}}(\Omega)^{d}$ such that both estimates of (4.11) hold. Taking $\boldsymbol{w}$ as a test function in the first relation of (4.4) yields:

$$
\|p\|_{\mathrm{L}^{2}(\Omega)}^{2}-\beta_{2}|p|_{\mathcal{G}}^{2} \leq \eta\left|\int_{\Omega} \boldsymbol{u}(\boldsymbol{x}) \cdot \boldsymbol{w}(\boldsymbol{x}) \mathrm{d} \boldsymbol{x}\right|+\nu\left|[\boldsymbol{u}, \boldsymbol{w}]_{\mathcal{D}}\right|+\left|\int_{\Omega} \boldsymbol{f}_{1}(\boldsymbol{x}) \cdot \boldsymbol{w}(\boldsymbol{x}) \mathrm{d} \boldsymbol{x}\right|+\left|\int_{\Omega} \boldsymbol{f}_{2}(\boldsymbol{x}) \cdot \boldsymbol{w}(\boldsymbol{x}) \mathrm{d} \boldsymbol{x}\right| .
$$

Using the discrete Poincaré inequality (3.6) and Young's inequality, we obtain:

$$
\begin{aligned}
\|p\|_{\mathrm{L}^{2}(\Omega)}^{2}-\beta_{2}|p|_{\mathcal{G}}^{2} \leq & \frac{1}{8}\|p\|_{\mathrm{L}^{2}(\Omega)}^{2}+2 \eta^{2} \operatorname{diam}(\Omega)^{2} \beta_{1}^{2}\|\boldsymbol{u}\|_{\mathrm{L}^{2}(\Omega)^{d}}^{2}+\frac{1}{8}\|p\|_{\mathrm{L}^{2}(\Omega)}^{2}+2 \nu^{2} \beta_{1}^{2}\|\boldsymbol{u}\|_{\mathcal{D}}^{2} \\
& +\frac{1}{8}\|p\|_{\mathrm{L}^{2}(\Omega)}^{2}+2 \operatorname{diam}(\Omega)^{2} \beta_{1}^{2}\left\|\boldsymbol{f}_{1}\right\|_{\mathrm{L}^{2}(\Omega)^{d}}^{2}+\frac{1}{8}\|p\|_{\mathrm{L}^{2}(\Omega)}^{2}+2 \beta_{1}^{2}\left\|\boldsymbol{f}_{2}\right\|_{-1, \mathcal{D}}^{2} .
\end{aligned}
$$

Let $\xi_{1}$ and $\xi_{2}$ be the positive parameters given by:

$$
\xi_{1}=\min \left[\frac{1}{8 \operatorname{diam}(\Omega)^{2} \beta_{1}^{2}}, \frac{1}{8 \beta_{1}^{2}}, \frac{1}{2 \beta_{2}}\right] \quad \xi_{2}=\min \left[\frac{1}{\eta}, \frac{1}{\nu}, \lambda\right] \xi_{1}=\frac{1}{\max [\eta, \nu, 1 / \lambda]} \xi_{1} .
$$

Note that $\xi_{1}$ only depends on $\theta$ and $\Omega$. From these definitions, we get by multiplying (4.15) by $\xi_{2}$ :

$$
\frac{\xi_{2}}{2}\|p\|_{\mathrm{L}^{2}(\Omega)}^{2}-\frac{\lambda}{2}|p|_{\mathcal{G}}^{2} \leq \frac{\eta}{4}\|\boldsymbol{u}\|_{\mathrm{L}^{2}(\Omega)^{d}}^{2}+\frac{\nu}{4}\|\boldsymbol{u}\|_{\mathcal{D}}^{2}+\frac{1}{4 \eta}\left\|\boldsymbol{f}_{1}\right\|_{\mathrm{L}^{2}(\Omega)^{d}}^{2}+\frac{1}{4 \nu}\left\|\boldsymbol{f}_{2}\right\|_{-1, \mathcal{D}}^{2}
$$

Then, taking $\boldsymbol{v}=\boldsymbol{u}$ in the first relation of (4.4) and $q=p$ in the second one and summing, we obtain, because the discrete gradient is the transpose of the discrete divergence:

$$
\eta\|\boldsymbol{u}\|_{\mathrm{L}^{2}(\Omega)^{d}}^{2}+\nu\|\boldsymbol{u}\|_{\mathcal{D}}^{2}+\lambda|p|_{\mathcal{G}}^{2}=\int_{\Omega} \boldsymbol{f}_{1}(\boldsymbol{x}) \cdot \boldsymbol{u}(\boldsymbol{x}) \mathrm{d} \boldsymbol{x}+\int_{\Omega} \boldsymbol{f}_{2}(\boldsymbol{x}) \cdot \boldsymbol{u}(\boldsymbol{x}) \mathrm{d} \boldsymbol{x}+\int_{\Omega} g(\boldsymbol{x}) p(\boldsymbol{x}) \mathrm{d} \boldsymbol{x} .
$$

By Young's inequality, we then have:

$$
\eta\|\boldsymbol{u}\|_{\mathrm{L}^{2}(\Omega)^{d}}^{2}+\nu\|\boldsymbol{u}\|_{\mathcal{D}}^{2}+\lambda|p|_{\mathcal{G}}^{2} \leq \frac{1}{\eta}\left\|\boldsymbol{f}_{1}\right\|_{\mathrm{L}^{2}(\Omega)^{d}}^{2}+\frac{\eta}{4}\|\boldsymbol{u}\|_{\mathrm{L}^{2}(\Omega)^{d}}^{2}+\frac{1}{\nu}\left\|\boldsymbol{f}_{2}\right\|_{-1, \mathcal{D}}^{2}+\frac{\nu}{4}\|\boldsymbol{u}\|_{\mathcal{D}}^{2}+\frac{1}{\xi_{2}}\left\|\left.g\right|_{\mathrm{L}^{2}(\Omega)} ^{2}+\frac{\xi_{2}}{4}\right\| p \|_{\mathrm{L}^{2}(\Omega)}^{2} .
$$

Summing (4.16) and (4.17) yields the desired result.

We can now state the existence and the uniqueness of a discrete solution to (4.4).

Corollary 4.6 (existence and uniqueness of a solution to the finite volume scheme). Under hypotheses (2.2)(2.4), let $\mathcal{D}$ be an admissible discretization of $\Omega$ in the sense of Definition 3.1. Let $\lambda \in(0,+\infty)$ be given. We suppose that the following compatibility condition holds (which is nothing more than the compatibility condition associated to the continuous problem):

$$
\int_{\Omega} g(\boldsymbol{x}) \mathrm{d} \boldsymbol{x}=0 .
$$

Then there exists a unique solution to (4.4). 
Proof. We define the following finite dimensional vector space:

$$
V=\left\{(\boldsymbol{u}, p) \in \mathrm{H}_{\mathcal{D}}(\Omega)^{d} \times \mathrm{H}_{\mathcal{D}}(\Omega) \text { such that } \int_{\Omega} p(\boldsymbol{x}) \mathrm{d} \boldsymbol{x}=0\right\} .
$$

Let $F$ be the linear mapping which associates to $(\boldsymbol{u}, p) \in V$ the pair $(\hat{\boldsymbol{u}}, \hat{p})$ defined by the following discrete variational identity:

$$
\mid \begin{array}{ll}
\eta \int_{\Omega} \boldsymbol{u}(\boldsymbol{x}) \cdot \boldsymbol{v}(\boldsymbol{x}) \mathrm{d} \boldsymbol{x}+\nu[\boldsymbol{u}, \boldsymbol{v}]_{\mathcal{D}}-\int_{\Omega} p(\boldsymbol{x}) \operatorname{div}_{\mathcal{D}} \boldsymbol{v}(\boldsymbol{x}) \mathrm{d} \boldsymbol{x}=\int_{\Omega} \hat{\boldsymbol{u}}(\boldsymbol{x}) \cdot \boldsymbol{v}(\boldsymbol{x}) \mathrm{d} \boldsymbol{x} & \forall \boldsymbol{v} \in \mathrm{H}_{\mathcal{D}}(\Omega)^{d} \\
\int_{\Omega} \operatorname{div}_{\mathcal{D}} \boldsymbol{u}(\boldsymbol{x}) q(\boldsymbol{x}) \mathrm{d} \boldsymbol{x}+\lambda\langle p, q\rangle_{\mathcal{G}}=\int_{\Omega} \hat{p}(\boldsymbol{x}) q(\boldsymbol{x}) \mathrm{d} \boldsymbol{x} & \forall q \in \mathrm{H}_{\mathcal{D}}(\Omega) .
\end{array}
$$

It is easy to check that this system has a unique solution (choosing as test functions the characteristic function of each control volume yields a linear system the matrix of which is the identity). Taking for $q$ the constant function equal to 1 (which lies in $\mathrm{H}_{\mathcal{D}}(\Omega)$ ) in the last relation, we check by conservativity that the integral of $\hat{p}$ over $\Omega$ is zero, which means that $(\hat{\boldsymbol{u}}, \hat{p}) \in V$. Theorem 4.4 then implies that the kernel of $F$ is reduced to $(0,0)$, which proves that the mapping $F$ is one to one from $V$ to $V$. As by assumption the integral of $g$ over $\Omega$ is zero, the pair of functions defined by the right hand side of (4.5) belongs to $V$, and this concludes the proof.

\subsection{Estimates of consistency residuals}

We define and estimate in this section the consistency residuals appearing in the convergence and error analysis of the scheme.

Let $v$ be a function from $\Omega$ to $\mathbb{R}$, the regularity of which will be made precise hereafter; for the moment, we only suppose that the following definitions make sense. For an internal edge $\sigma=K \mid L$, we set:

$$
\begin{aligned}
& R_{\Delta, K \mid L}(v)=\frac{1}{d_{K \mid L}}\left[v\left(\boldsymbol{x}_{L}\right)-v\left(\boldsymbol{x}_{K}\right)\right]-\frac{1}{m_{K \mid L}} \int_{K \mid L} \nabla v(\boldsymbol{x}) \cdot \boldsymbol{n}_{K \mid L} \mathrm{~d} \gamma(\boldsymbol{x}) \\
& R_{\text {grad }, \mathcal{M}, K \mid L}(v)=\frac{d_{K, K \mid L}}{d_{K \mid L}} P_{\mathcal{M}}(v)_{K}+\frac{d_{L, K \mid L}}{d_{K \mid L}} P_{\mathcal{M}}(v)_{L}-\frac{1}{m_{K \mid L}} \int_{K \mid L} v(\boldsymbol{x}) \mathrm{d} \gamma(\boldsymbol{x}) \\
& R_{\text {grad }, \mathcal{G}, K \mid L}(v)=\frac{d_{K, K \mid L}}{d_{K \mid L}} P_{\mathcal{G}}(v)_{K}+\frac{d_{L, K \mid L}}{d_{K \mid L}} P_{\mathcal{G}}(v)_{L}-\frac{1}{m_{K \mid L}} \int_{K \mid L} v(\boldsymbol{x}) \mathrm{d} \gamma(\boldsymbol{x}) \\
& R_{\text {div }, K \mid L}(v)=\frac{d_{L, K \mid L}}{d_{K \mid L}} v\left(\boldsymbol{x}_{K}\right)+\frac{d_{K, K \mid L}}{d_{K \mid L}} v\left(\boldsymbol{x}_{L}\right)-\frac{1}{m_{K \mid L}} \int_{K \mid L} v(\boldsymbol{x}) \mathrm{d} \gamma(\boldsymbol{x}) \\
& R_{\text {stab }, \mathcal{M}, K \mid L}(v)=\left(h_{K}+h_{L}\right)\left(P_{\mathcal{M}}(v)_{L}-P_{\mathcal{M}}(v)_{K}\right)
\end{aligned}
$$

and, for an external edge $\sigma, \sigma \in \mathcal{E}(K)$ :

$$
\begin{aligned}
& R_{\Delta, \sigma}(v)=-\frac{1}{d_{K, \sigma}} v\left(\boldsymbol{x}_{K}\right)-\frac{1}{m_{\sigma}} \int_{\sigma} \nabla v(\boldsymbol{x}) \cdot \boldsymbol{n}_{\sigma} \mathrm{d} \gamma(\boldsymbol{x}) \\
& R_{\operatorname{grad}, \mathcal{M}, \sigma}(v)=P_{\mathcal{M}}(v)_{K}-\frac{1}{m_{\sigma}} \int_{\sigma} v(\boldsymbol{x}) \mathrm{d} \gamma(\boldsymbol{x}) \\
& R_{\operatorname{grad}, \mathcal{G}, \sigma}(v)=P_{\mathcal{G}}(v)_{K}-\frac{1}{m_{\sigma}} \int_{\sigma} v(\boldsymbol{x}) \mathrm{d} \gamma(\boldsymbol{x}) \\
& R_{\operatorname{div}, \sigma}(v)=R_{\mathrm{stab}, \mathcal{M}, \sigma}(v)=0 .
\end{aligned}
$$


In addition, we define, for any control volume $K$ of the mesh:

$$
R_{\mathrm{o}, K}(v)=v\left(\boldsymbol{x}_{K}\right)-\frac{1}{m_{K}} \int_{K} v(\boldsymbol{x}) \mathrm{d} \boldsymbol{x} .
$$

The consistency residuals of the scheme are now defined as follows:

$$
\begin{array}{ll}
\forall \boldsymbol{v} \in \mathrm{H}^{2}(\Omega)^{d}, \boldsymbol{R}_{\mathrm{o}}(\boldsymbol{v}) \in \mathrm{H}_{\mathcal{D}}(\Omega)^{d}, \quad\left(\boldsymbol{R}_{\mathrm{o}}(\boldsymbol{v})^{(i)}\right)_{K}=R_{\mathrm{o}, K}\left(\boldsymbol{v}^{(i)}\right), \quad i=1, \ldots, d \\
\forall \boldsymbol{v} \in \mathrm{H}^{2}(\Omega)^{d}, \boldsymbol{R}_{\Delta}(\boldsymbol{v}) \in \mathrm{H}_{\mathcal{D}}(\Omega)^{d}, \quad\left(\boldsymbol{R}_{\Delta}(\boldsymbol{v})^{(i)}\right)_{K}=\frac{1}{m_{K}} \sum_{\sigma \in \mathcal{E}(K)} m_{\sigma} R_{\Delta, \sigma}\left(\boldsymbol{v}^{(i)}\right), \quad i=1, \ldots, d \\
\forall v \in \mathrm{L}^{2}(\Omega), \boldsymbol{R}_{\mathrm{grad}, \mathcal{M}}(v) \in \mathrm{H}_{\mathcal{D}}(\Omega)^{d}, & \left(\boldsymbol{R}_{\mathrm{grad}, \mathcal{M}}(v)\right)_{K}=\frac{1}{m_{K}} \sum_{\sigma \in \mathcal{E}(K)} m_{\sigma} R_{\mathrm{grad}, \mathcal{M}, \sigma}(v) \boldsymbol{n}_{\sigma} \\
\forall v \in \mathrm{L}^{2}(\Omega), \boldsymbol{R}_{\mathrm{grad}, \mathcal{G}}(v) \in \mathrm{H}_{\mathcal{D}}(\Omega)^{d}, & \left(\boldsymbol{R}_{\mathrm{grad}, \mathcal{G}}(v)\right)_{K}=\frac{1}{m_{K}} \sum_{\sigma \in \mathcal{E}(K)} m_{\sigma} R_{\mathrm{grad}, \mathcal{G}, \sigma}(v) \boldsymbol{n}_{\sigma} \\
\forall \boldsymbol{v} \in \mathrm{H}^{2}(\Omega)^{d}, R_{\mathrm{div}}(\boldsymbol{v}) \in \mathrm{H}_{\mathcal{D}}(\Omega), & \left(R_{\mathrm{div}}(\boldsymbol{v})\right)_{K}=\frac{1}{m_{K}} \sum_{\sigma \in \mathcal{E}(K)} m_{\sigma}\left[\sum_{i=1}^{d} R_{\mathrm{div}, \sigma}\left(\boldsymbol{v}^{(i)}\right) \boldsymbol{e}_{i}\right] \cdot \boldsymbol{n}_{\sigma} \\
\forall v \in \mathrm{L}^{2}(\Omega), R_{\mathrm{stab}, \mathcal{M}}(v) \in \mathrm{H}_{\mathcal{D}}(\Omega), \quad\left(R_{\mathrm{stab}, \mathcal{M}}(v)\right)_{K}=\frac{1}{m_{K}} \sum_{\sigma \in \mathcal{E}(K)} m_{\sigma} R_{\mathrm{stab}, \mathcal{M}, \sigma}(v) .
\end{array}
$$

The following theorem gathers the estimates of the residuals which will be useful in the convergence and error analysis.

Theorem 4.7 (estimates of the consistency residuals). Let assumption (2.2) hold, $\mathcal{D}$ be an admissible discretization of $\Omega$ in the sense of Definition 3.1 and $\theta>0$ be such that $\operatorname{regul}(\mathcal{D}, \mathcal{G})>\theta$. Let $(\boldsymbol{v}, q) \in\left(\mathrm{H}^{2}(\Omega)^{d} \cap \mathrm{H}_{0}^{1}(\Omega)^{d}\right) \times$ $\mathrm{H}^{1}(\Omega)$. Then the following bounds hold:

$$
\begin{aligned}
\left\|\boldsymbol{R}_{\Delta}(\boldsymbol{v})\right\|_{-1, \mathcal{D}} & \leq c_{\Delta} h_{\mathcal{M}}|\boldsymbol{v}|_{\mathrm{H}^{2}(\Omega)^{d}} \\
\left\|\boldsymbol{R}_{\text {grad }, \mathcal{M}}(q)\right\|_{-1, \mathcal{D}} & \leq c_{\mathrm{grad}, \mathcal{M}} h_{\mathcal{M}}|q|_{\mathrm{H}^{1}(\Omega)} \\
\left\|\boldsymbol{R}_{\operatorname{grad}, \mathcal{G}}(q)\right\|_{-1, \mathcal{D}} & \leq c_{\mathrm{grad}, \mathcal{G}} h_{\mathcal{G}}|q|_{\mathrm{H}^{1}(\Omega)} \\
\left\|\boldsymbol{R}_{\mathrm{O}}(\boldsymbol{v})\right\|_{\mathrm{L}^{2}(\Omega)^{d}} & \leq c_{\mathrm{O}} h_{\mathcal{M}}\|\boldsymbol{v}\|_{\mathrm{H}^{2}(\Omega)^{d}} \\
\left\|R_{\mathrm{stab}, \mathcal{M}}(q)\right\|_{\mathrm{L}^{2}(\Omega)} & \leq c_{\mathrm{stab}} h_{\mathcal{M}}|q|_{\mathrm{H}^{1}(\Omega)}
\end{aligned}
$$

where $c_{\Delta}, c_{\mathrm{grad}, \mathcal{M}}, c_{\mathrm{grad}, \mathcal{G}}, c_{\mathrm{O}}$ and $c_{\mathrm{stab}}$ only depend on $d, \Omega$ and $\theta$ and $|\cdot|_{\mathrm{H}^{1}(\Omega)},\|\cdot\|_{\mathrm{H}^{2}(\Omega)}$ and $|\cdot|_{\mathrm{H}^{2}(\Omega)}$ stand for the usual Sobolev $\mathrm{H}^{1}$ seminorm and $\mathrm{H}^{2}$ norm and seminorm respectively.

If in addition the mesh is super-admissible in the sense of Definition 3.2, then:

$$
\left\|R_{\mathrm{div}}(\boldsymbol{v})\right\|_{\mathrm{L}^{2}(\Omega)} \leq c_{\mathrm{div}} h_{\mathcal{M}}|\boldsymbol{v}|_{\mathrm{H}^{2}(\Omega)^{d}}
$$

where $c_{\mathrm{div}}$ only depends on $d, \Omega$ and $\theta$.

Proof. $\mathrm{H}_{\mathcal{D}}^{-1}$ estimates (4.18)-(4.20)

The proof of the three discrete $\mathrm{H}^{-1}$ estimates (4.18), (4.19) and (4.20) being similar, we shall describe the general technique and then apply it only to obtain (4.20). Consider a consistency residual $R \in \mathrm{H}_{\mathcal{D}}(\Omega)$ under the general form:

$$
R_{K}=\frac{1}{m_{K}} \sum_{\sigma \in \mathcal{E}(K)} m_{\sigma} R_{\sigma}
$$


with, for any internal edge $\sigma=K \mid L, R_{K \mid L}=-R_{L \mid K}$. Let $v$ be a function of $\mathrm{H}_{\mathcal{D}}$. Then we have:

$$
\int_{\Omega} R(\boldsymbol{x}) v(\boldsymbol{x}) \mathrm{d} \boldsymbol{x}=\sum_{K \in \mathcal{M}} m_{K} R_{K} v_{K}=\sum_{K \in \mathcal{M}} v_{K} \sum_{\sigma \in \mathcal{E}(K)} m_{\sigma} R_{\sigma} .
$$

Reordering the sums, we get:

$$
\int_{\Omega} R(\boldsymbol{x}) v(\boldsymbol{x}) \mathrm{d} \boldsymbol{x}=\sum_{\sigma \in \mathcal{E}_{\text {int }}, \sigma=K \mid L} m_{\sigma} R_{\sigma}\left(v_{K}-v_{L}\right)+\sum_{\sigma \in \mathcal{E}_{\text {ext }}, \sigma \in \mathcal{E}(K)} m_{\sigma} R_{\sigma} v_{K}
$$

and, by the (discrete) Cauchy-Schwarz inequality:

$$
\begin{aligned}
\left|\int_{\Omega} R(\boldsymbol{x}) v(\boldsymbol{x}) \mathrm{d} \boldsymbol{x}\right| & \leq\left[\sum_{\sigma \in \mathcal{E}} d_{\sigma} m_{\sigma} R_{\sigma}^{2}\right]^{1 / 2}\left[\sum_{\sigma \in \mathcal{E}_{\text {int }}, \sigma=K \mid L} \frac{m_{\sigma}}{d_{\sigma}}\left(v_{K}-v_{L}\right)^{2}+\sum_{\sigma \in \mathcal{E}_{\text {ext }}, \sigma \in \mathcal{E}(K)} \frac{m_{\sigma}}{d_{\sigma}} v_{K}^{2}\right]^{1 / 2} \\
& \leq\left[\sum_{\sigma \in \mathcal{E}} d_{\sigma} m_{\sigma} R_{\sigma}^{2}\right]^{1 / 2}\|v\|_{\mathcal{D}} .
\end{aligned}
$$

In order to obtain an estimate in the $\|\cdot\|_{-1, \mathcal{D}}$ norm of $R$, there only remains to bound the sum $\sum_{\sigma \in \mathcal{E}} d_{\sigma} m_{\sigma} R_{\sigma}^{2}$, which must be done thanks to elementary (i.e. local to each edge) residual bounds, stated here in Lemma A.2 or Corollary A.4. Let us now, for instance, apply this technique to the estimate of $\boldsymbol{R}_{\mathrm{grad}, \mathcal{G}}$. Let $q$ be a function of $\mathrm{H}^{1}(\Omega)$ and $\boldsymbol{v}$ a function of $\mathrm{H}_{\mathcal{D}}(\Omega)^{d}$. By definition, we have:

$$
\int_{\Omega} \boldsymbol{R}_{\mathrm{grad}, \mathcal{G}}(q)(\boldsymbol{x}) \cdot \boldsymbol{v}(\boldsymbol{x}) \mathrm{d} \boldsymbol{x}=\sum_{K \in \mathcal{M}} m_{K} \frac{1}{m_{K}} \sum_{\sigma \in \mathcal{E}(K)} m_{\sigma} R_{\mathrm{grad}, \mathcal{G}, \sigma}(q) \boldsymbol{n}_{\sigma} \cdot \boldsymbol{v}_{K}
$$

By the computation described above, we thus get:

$$
\begin{aligned}
&\left|\int_{\Omega} \boldsymbol{R}_{\mathrm{grad}, \mathcal{G}}(q)(\boldsymbol{x}) \cdot \boldsymbol{v}(\boldsymbol{x}) \mathrm{d} \boldsymbol{x}\right| \leq\left[\sum_{\sigma \in \mathcal{E}} d_{\sigma} m_{\sigma} R_{\mathrm{grad}, \mathcal{G}, \sigma}(q)^{2}\right]^{1 / 2} \\
& \times\left[\sum_{\sigma \in \mathcal{E}_{\text {int }}, \sigma=K \mid L} \frac{m_{\sigma}}{d_{\sigma}}\left[\left(\boldsymbol{v}_{K}-\boldsymbol{v}_{L}\right) \cdot \boldsymbol{n}_{\sigma}\right]^{2}+\sum_{\sigma \in \mathcal{E}_{\text {ext }}, \sigma \in \mathcal{E}(K)} \frac{m_{\sigma}}{d_{\sigma}}\left[\boldsymbol{v}_{K} \cdot \boldsymbol{n}_{\sigma}\right]^{2}\right]^{1 / 2} .
\end{aligned}
$$

Now, since $\forall \boldsymbol{a} \in \mathbb{R}^{d}, \boldsymbol{a} \cdot \boldsymbol{n} \leq|\boldsymbol{a}|$, we get:

$$
\left|\int_{\Omega} \boldsymbol{R}_{\mathrm{grad}, \mathcal{G}}(q)(\boldsymbol{x}) \cdot \boldsymbol{v}(\boldsymbol{x}) \mathrm{d} \boldsymbol{x}\right| \leq\left[\sum_{\sigma \in \mathcal{E}} d_{\sigma} m_{\sigma} R_{\mathrm{grad}, \mathcal{G}, \sigma}(q)^{2}\right]^{1 / 2}\|\boldsymbol{v}\|_{\mathcal{D}} .
$$

Using the elementary residual estimate (A.12) of Corollary A.4 in the Appendix yields:

$$
\left|\int_{\Omega} \boldsymbol{R}_{\mathrm{grad}, \mathcal{G}}(q)(\boldsymbol{x}) \cdot \boldsymbol{v}(\boldsymbol{x}) \mathrm{d} \boldsymbol{x}\right| \leq c\|\boldsymbol{v}\|_{\mathcal{D}}\left[\sum_{\sigma \in \mathcal{E}} d_{\sigma} m_{\sigma} \frac{h_{\mathcal{D}}}{m_{\sigma}}|q|_{\mathrm{H}^{1}\left(C_{\sigma}\right)}^{2}\right]^{1 / 2}
$$

where, if $\sigma \in \mathcal{E}_{\text {int }}, \sigma=K \mid L, C_{\sigma}$ stands for the union of the clusters including $K$ and $L$ (i.e. $C_{\sigma}=\cup_{M \in\left(G_{K} \cup G_{L}\right)} M$ ) and, if $\sigma \in \mathcal{E}_{\text {ext }}, \sigma \in \mathcal{E}(K), C_{\sigma}$ stands for the cluster including $K$ (i.e. $C_{\sigma}=\cup_{M \in G_{K}} M$ ). Thanks to the regularity 
assumption on the mesh, for a given control volume $K$, the number of domains $C_{\sigma}$ including $K$ is bounded by a constant $c_{\mathrm{cl}}$; the term $|q|_{\mathrm{H}^{1}(K)}^{2}$ thus also appears in the above summation only $c_{\mathrm{cl}}$ times and we obtain:

$$
\left|\int_{\Omega} \boldsymbol{R}_{\mathrm{grad}, \mathcal{G}}(q)(\boldsymbol{x}) \cdot \boldsymbol{v}(\boldsymbol{x}) \mathrm{d} \boldsymbol{x}\right| \leq c\|\boldsymbol{v}\|_{\mathcal{D}} c_{\mathrm{cl}}\left(\max _{\sigma \in \mathcal{E}} d_{\sigma} h_{\mathcal{D}}\right)^{1 / 2}|q|_{\mathrm{H}^{1}(\Omega)}
$$

which yields the desired estimate for $\boldsymbol{R}_{\mathrm{grad}, \mathcal{G}}$.

$\mathrm{L}^{2}$ estimates (4.21)-(4.23)

Estimates of the consistency residuals in $\mathrm{L}^{2}$ are obtained in a straightforward way from the bounds of the elementary consistency residuals estimates of Lemma A.2 or Corollary A.4 in the Appendix. As an example, we detail here the bound (4.23); the bounds (4.21) and (4.22) are obtained in a similar way.

Let $\boldsymbol{v}($.$) be a function of \mathrm{H}^{2}(\Omega)^{d}$, and the mesh be super-admissible. By definition, we have:

$$
\left\|R_{\operatorname{div}}(\boldsymbol{v})\right\|_{\mathrm{L}^{2}(\Omega)}^{2}=\sum_{K \in \mathcal{M}} m_{K}\left[\frac{1}{m_{K}} \sum_{\sigma \in \mathcal{E}(K)} m_{\sigma}\left[\sum_{i=1}^{d} R_{\mathrm{div}, \sigma}\left(\boldsymbol{v}^{(i)}\right) \boldsymbol{e}_{i}\right] \cdot \boldsymbol{n}_{\sigma}\right]^{2} .
$$

Expanding the sum, we have:

$$
\left[\sum_{\sigma \in \mathcal{E}(K)} m_{\sigma}\left[\sum_{i=1}^{d} R_{\operatorname{div}, \sigma}\left(\boldsymbol{v}^{(i)}\right) \boldsymbol{e}_{i}\right] \cdot \boldsymbol{n}_{\sigma}\right]^{2} \leq c \sum_{\sigma \in \mathcal{E}(K)} m_{\sigma}^{2} \sum_{i=1}^{d} R_{\operatorname{div}, \sigma}\left(\boldsymbol{v}^{(i)}\right)^{2}
$$

where $c$ only depends on the number of edges of a control volume and on the space dimension $d$. Since, by definition, $R_{\mathrm{div}, \sigma}$ vanishes on external boundaries, we then get:

$$
\left\|R_{\mathrm{div}}(\boldsymbol{v})\right\|_{\mathrm{L}^{2}(\Omega)}^{2} \leq c \sum_{\sigma \in \mathcal{E}_{\mathrm{int}}, \sigma=K \mid L}\left(\frac{1}{m_{K}}+\frac{1}{m_{L}}\right) m_{\sigma}^{2} \sum_{i=1}^{d} R_{\mathrm{div}, \sigma}\left(\boldsymbol{v}^{(i)}\right)^{2} .
$$

The bound (A.6) thus yields:

$$
\left\|R_{\mathrm{div}}(\boldsymbol{v})\right\|_{\mathrm{L}^{2}(\Omega)}^{2} \leq c \sum_{\sigma \in \mathcal{E}_{\mathrm{int}}, \sigma=K \mid L}\left(\frac{1}{m_{K}}+\frac{1}{m_{L}}\right) m_{\sigma}^{2} \sum_{i=1}^{d} \frac{\left(h_{K}+h_{L}\right)^{4}}{m_{\sigma} \mathrm{d}_{\sigma}}\left|\boldsymbol{v}^{(i)}\right|_{\mathrm{H}^{2}\left(D_{\sigma}\right)}^{2} .
$$

The estimate of $R_{\text {div }}(\boldsymbol{v})$ is then completed using the regularity assumptions for the mesh.

\subsection{Convergence of the scheme}

The aim of this section is to prove the convergence of the scheme (4.4) to the unique solution to the generalized Stokes problem without any regularity assumption for this latter. This result is stated in the following theorem.

Theorem 4.8 (convergence for the generalized Stokes problem). Under hypotheses (2.2)-(2.4), let $(\overline{\boldsymbol{u}}, \bar{p})$ be the unique weak solution to the Stokes problem (2.7) in the sense of Definition 2.2. Let $\theta>0$ be given and let $\left(\mathcal{D}^{(m)}\right)_{m \in \mathbb{N}}$ be a sequence of super-admissible discretizations of $\Omega$, in the sense of Definitions 3.1 and 3.2, such that, for all $m \in \mathbb{N}$, $\operatorname{regul}\left(\mathcal{D}^{(m)}, \mathcal{G}^{(m)}\right) \geq \theta$ and such that $\lim _{m \rightarrow+\infty} h_{\mathcal{D}}^{(m)}=0$. We denote by $\left(\boldsymbol{u}^{(m)}, p^{(m)}\right) \in$ $\mathrm{H}_{\mathcal{D}^{(m)}}(\Omega)^{d} \times \mathrm{H}_{\mathcal{D}^{(m)}}(\Omega)$ the unique solution to (4.4) with the discretization $\mathcal{D}^{(m)}$, where the parameter $\lambda \in(0,+\infty)$ is independent of $m$. Then the sequence $\left(\boldsymbol{u}^{(m)}\right)_{m \in \mathbb{N}}$ converges to $\overline{\boldsymbol{u}}$ in $\left(\mathrm{L}^{2}(\Omega)\right)^{d}$ and $\left(p^{(m)}\right)_{m \in \mathbb{N}}$ weakly converges to $\bar{p}$ in $\mathrm{L}^{2}(\Omega)$. 
Proof. We first prove, under the hypotheses and with the notations of the above theorem, the existence of a subsequence of $\left(\mathcal{D}^{(m)}\right)_{m \in \mathbb{N}}$ such that the corresponding sequence $\left(\boldsymbol{u}^{(m)}\right)_{m \in \mathbb{N}}$ converges in $\left(\mathrm{L}^{2}(\Omega)\right)^{d}$ to a function $\overline{\boldsymbol{u}} \in \mathrm{H}_{0}^{1}(\Omega)^{d}$ and the sequence $\left(p^{(m)}\right)_{m \in \mathbb{N}}$ weakly converges in $\mathrm{L}^{2}(\Omega)$ to a function $\bar{p} \in \mathrm{L}^{2}(\Omega)$, as $m \rightarrow+\infty$; then we show that the pair $(\overline{\boldsymbol{u}}, \bar{p})$ is a weak solution to the generalized Stokes problem, in the sense of Definition 2.2; finally, the convergence holds for the whole sequence thanks to the uniqueness of the solution to this continuous problem.

By the fact that $\left\|\boldsymbol{u}^{(m)}\right\|_{\mathcal{D}^{(m)}}$ is bounded independently of $m$ by the stability estimate (4.14), we obtain (see [11], Lem. 9.3, p. 770) the following estimate on the translates of $\boldsymbol{u}^{(m)}$. For all $m \in \mathbb{N}$, there exists $c$, only depending on $d, \Omega, \nu, \boldsymbol{f}, g$ and $\theta$ such that:

$$
\int_{\Omega}\left[\boldsymbol{u}^{(m, i)}(\boldsymbol{x}+\boldsymbol{\xi})-\boldsymbol{u}^{(m, i)}(\boldsymbol{x})\right]^{2} \mathrm{~d} \boldsymbol{x} \leq c|\boldsymbol{\xi}|\left[|\boldsymbol{\xi}|+4 h^{(m)}\right], \quad \text { for } i=1, \ldots, d, \quad \forall \boldsymbol{\xi} \in \mathbb{R}^{d},
$$

where $\boldsymbol{u}^{(m, i)}$ denotes the $i$-th component of $\boldsymbol{u}^{(m)}$, extended by zero outside $\Omega$, and $h^{(m)}$ stands for the size of the discretization $\mathcal{D}^{(m)}$. We then apply Kolmogorov's theorem (e.g. [11], Thm. 14.1, p. 833), and obtain the existence of a subsequence of $\left(\mathcal{D}^{(m)}\right)_{m \in \mathbb{N}}$ and of $\overline{\boldsymbol{u}} \in \mathrm{L}^{2}(\Omega)^{d}$ such that $\left(\boldsymbol{u}^{(m)}\right)_{m \in \mathbb{N}}$ converges to $\overline{\boldsymbol{u}}$ in $\mathrm{L}^{2}(\Omega)^{d}$. Moreover, still using estimate (4.24), this limit $\overline{\boldsymbol{u}}$ is shown to belong to $\mathrm{H}_{0}^{1}(\Omega)^{d}[11]$, Theorem 14.2, p. 833.

In addition, thanks to the fact that $\left\|p^{(m)}\right\|_{\mathrm{L}^{2}(\Omega)}$ is bounded independently of $m$ by the same bound (4.14), we extract from this subsequence another one (still denoted $\left(\mathcal{D}^{(m)}\right)_{m \in \mathbb{N}}$ ) such that $\left(p^{(m)}\right)_{m \in \mathbb{N}}$ weakly converges to some function $\bar{p}$ in $\mathrm{L}^{2}(\Omega)$.

In order to prove the convergence of the scheme, we now must show that $(\overline{\boldsymbol{u}}, \bar{p})$ is the solution to $(2.8)$. By density, it is sufficient to prove that this variational problem is satisfied for any test function in $C_{c}^{\infty}(\Omega)^{d}$. This will be proved by passing to the limit in the scheme. We thus take $\varphi \in C_{c}^{\infty}(\Omega)^{d}$, and suppose that $m$ is large enough and thus $h_{\mathcal{D}}^{(m)}$ is small enough to ensure that, for all $K \in \mathcal{M}$ such that $K \cap \operatorname{support}(\varphi) \neq \emptyset$, $\partial K \cap \partial \Omega=\emptyset$. Let us take $\boldsymbol{v}=P_{\mathcal{D}^{(m)}} \boldsymbol{\varphi}$ in (4.4), which yields:

$$
\begin{aligned}
\eta \int_{\Omega} \boldsymbol{u}^{(m)}(\boldsymbol{x}) \cdot P_{\mathcal{D}^{(m)}} \boldsymbol{\varphi}(\boldsymbol{x}) & \mathrm{d} \boldsymbol{x}+\nu\left[\boldsymbol{u}^{(m)}, P_{\mathcal{D}^{(m)}} \boldsymbol{\varphi}\right]_{\mathcal{D}^{(m)}} \\
& -\int_{\Omega} p^{(m)}(\boldsymbol{x}) \operatorname{div}_{\mathcal{D}^{(m)}}\left(P_{\mathcal{D}^{(m)}} \boldsymbol{\varphi}\right)(\boldsymbol{x}) \mathrm{d} \boldsymbol{x}=\int_{\Omega} \boldsymbol{f}(\boldsymbol{x}) \cdot P_{\mathcal{D}^{(m)}} \boldsymbol{\varphi}(\boldsymbol{x}) \mathrm{d} \boldsymbol{x} .
\end{aligned}
$$

We write this latter relation as follows:

$$
\eta \int_{\Omega} \boldsymbol{u}^{(m)}(\boldsymbol{x}) \cdot \boldsymbol{\varphi}(\boldsymbol{x}) \mathrm{d} \boldsymbol{x}-\nu \int_{\Omega} \boldsymbol{u}^{(m)} \Delta \boldsymbol{\varphi} \mathrm{d} \boldsymbol{x}-\int_{\Omega} p^{(m)}(\boldsymbol{x}) \operatorname{div} \boldsymbol{\varphi}(\boldsymbol{x}) \mathrm{d} \boldsymbol{x}+R_{1}=\int_{\Omega} \boldsymbol{f}(\boldsymbol{x}) \cdot \boldsymbol{\varphi}(\boldsymbol{x}) \mathrm{d} \boldsymbol{x}+R_{2}
$$

where $R_{1}$ is the sum of three terms $R_{1}=T_{\mathrm{o}}+T_{\Delta}+T_{\text {grad }}$ which are defined hereafter, together with $R_{2}$. We first have:

$$
T_{\mathrm{o}}=\eta \int_{\Omega} \boldsymbol{u}^{(m)}(\boldsymbol{x}) \cdot\left(P_{\mathcal{D}^{(m)}} \boldsymbol{\varphi}(\boldsymbol{x})-\boldsymbol{\varphi}(\boldsymbol{x})\right) \mathrm{d} \boldsymbol{x}
$$

and thus, by the Cauchy-Schwarz inequality and by Theorem 4.7:

$$
\left|T_{\mathrm{o}}\right| \leq \eta\left\|\boldsymbol{u}^{(m)}\right\|_{\mathrm{L}^{2}(\Omega)^{d}}\left\|\boldsymbol{R}_{\mathrm{O}}(\boldsymbol{\varphi})\right\|_{\mathrm{L}^{2}(\Omega)^{d}} \leq c_{1}(\boldsymbol{\varphi}) h^{(m)}\left\|\boldsymbol{u}^{(m)}\right\|_{\mathrm{L}^{2}(\Omega)^{d}} .
$$

Using as in equation (4.5) the classical finite volume expression for the diffusion term, $T_{\Delta}$ reads $T_{\Delta}=\sum_{i=1}^{d} T_{\Delta}^{(i)}$ with:

$$
T_{\Delta}^{(i)}=-\nu \sum_{K \in \mathcal{M}} \boldsymbol{\varphi}^{(i)}\left(\boldsymbol{x}_{K}\right) \sum_{\sigma \in \mathcal{E}(K), \sigma=K \mid L} \frac{m_{\sigma}}{d_{\sigma}}\left(\boldsymbol{u}_{L}^{(m, i)}-\boldsymbol{u}_{K}^{(m, i)}\right)+\nu \sum_{K \in \mathcal{M}} \boldsymbol{u}_{K}^{(m, i)} \int_{K} \Delta \boldsymbol{\varphi}^{(i)}(\boldsymbol{x}) \mathrm{d} \boldsymbol{x} .
$$


Reordering the sums, we get:

$$
T_{\Delta}^{(i)}=-\nu \sum_{K \in \mathcal{M}} \boldsymbol{u}_{K}^{(m, i)} \sum_{\sigma \in \mathcal{E}(K), \sigma=K \mid L} \frac{m_{\sigma}}{d_{\sigma}}\left(\boldsymbol{\varphi}^{(i)}\left(\boldsymbol{x}_{L}\right)-\boldsymbol{\varphi}^{(i)}\left(\boldsymbol{x}_{K}\right)\right)-\int_{\sigma} \boldsymbol{\nabla} \boldsymbol{\varphi}^{(i)}(\boldsymbol{x}) \cdot \boldsymbol{n}_{\sigma} \mathrm{d} \gamma(\boldsymbol{x}) .
$$

Using the definition of $R_{\Delta, K \mid L}$, this relation equivalently reads:

$$
T_{\Delta}^{(i)}=-\nu \sum_{K \in \mathcal{M}} \boldsymbol{u}_{K}^{(m, i)} \sum_{\sigma \in \mathcal{E}(K), \sigma=K \mid L} m_{\sigma} R_{\Delta, K \mid L}\left(\varphi^{(i)}\right)=-\nu \int_{\Omega} \boldsymbol{u}^{(m, i)} \boldsymbol{R}_{\Delta}(\varphi)^{(i)} \mathrm{d} \boldsymbol{x}
$$

By the definition of the $\|\cdot\|_{-1, \mathcal{D}}$ norm, then by Theorem 4.7 , we thus have:

$$
\left|T_{\Delta}\right| \leq \nu\left\|\boldsymbol{u}^{(m)}\right\|_{\mathcal{D}^{(m)}}\left\|\boldsymbol{R}_{\Delta}(\boldsymbol{\varphi})\right\|_{-1, \mathcal{D}^{(m)}} \leq c_{2}(\boldsymbol{\varphi}) h^{(m)}\left\|\boldsymbol{u}^{(m)}\right\|_{\mathcal{D}}
$$

The third term is defined and bounded as follows:

$$
\left|T_{\text {grad }}\right|=\left|\int_{\Omega} p^{(m)}(\boldsymbol{x}) R_{\mathrm{div}}(\boldsymbol{\varphi})(\boldsymbol{x}) \mathrm{d} \boldsymbol{x}\right| \leq c_{3}(\boldsymbol{\varphi}) h^{(m)}\left\|p^{(m)}\right\|_{\mathrm{L}^{2}(\Omega)}
$$

and, finally:

$$
\left|R_{2}\right|=\left|\int_{\Omega} \boldsymbol{f}(\boldsymbol{x}) \boldsymbol{R}_{\mathrm{o}}(\boldsymbol{\varphi})(\boldsymbol{x}) \mathrm{d} \boldsymbol{x}\right| \leq c_{4}(\boldsymbol{\varphi}) h^{(m)}\|\boldsymbol{f}\|_{\mathrm{L}^{2}(\Omega)} .
$$

We thus obtain that both $R_{1}$ and $R_{2}$ tend to zero when $m$ tends to $+\infty$, and passing to the limit in (4.25) yields that the first equation of the generalized Stokes problem is satisfied. The last step in proving that $(\overline{\boldsymbol{u}}, \bar{p})$ is the weak solution to the Stokes problem is to show that $\operatorname{div}(\overline{\boldsymbol{u}})(\boldsymbol{x})=g(\boldsymbol{x})$ for a.e. $\boldsymbol{x} \in \Omega$. Let us take now $\varphi \in C_{c}^{\infty}(\Omega)$ and $q=P_{\mathcal{G}^{(m)}} \varphi$ in the second equation of (4.4), to obtain:

$$
\int_{\Omega}\left(\operatorname{div}_{\mathcal{D}} \boldsymbol{u}^{(m)}\right)(\boldsymbol{x})\left(P_{\mathcal{G}^{(m)}} \varphi\right)(\boldsymbol{x}) \mathrm{d} \boldsymbol{x}+\lambda\left\langle p^{(m)}, P_{\mathcal{G}^{(m)}} \varphi\right\rangle_{\mathcal{G}^{(m)}}=\int_{\Omega} g(\boldsymbol{x})\left(P_{\mathcal{G}^{(m)}} \varphi\right)(\boldsymbol{x}) \mathrm{d} \boldsymbol{x}
$$

As $P_{\mathcal{G}^{(m)}} \varphi$ is constant over each cluster, the stabilization term vanishes. Using the fact that the discrete divergence is the transpose of the discrete gradient, we get:

$$
\int_{\Omega}\left(\operatorname{div}_{\mathcal{D}} \boldsymbol{u}^{(m)}\right)(\boldsymbol{x})\left(P_{\mathcal{G}^{(m)}} \varphi\right)(\boldsymbol{x}) \mathrm{d} \boldsymbol{x}=-\int_{\Omega} \boldsymbol{u}^{(m)}(\boldsymbol{x}) \cdot\left(\nabla_{\mathcal{D}} P_{\mathcal{G}}(m) \varphi\right)(\boldsymbol{x}) \mathrm{d} \boldsymbol{x}=-\int_{\Omega} \boldsymbol{u}^{(m)}(\boldsymbol{x}) \cdot(\boldsymbol{\nabla} \varphi)(\boldsymbol{x}) \mathrm{d} \boldsymbol{x}+R_{3}
$$

where $R_{3}$ reads:

$$
R_{3}=-\sum_{K \in \mathcal{M}} \boldsymbol{u}_{K}^{(m)} \cdot \sum_{\sigma \in \mathcal{E}(K)} m_{\sigma} R_{\mathrm{grad}, \mathcal{G}, \sigma}(\varphi) \boldsymbol{n}_{\sigma}=-\int_{\Omega} \boldsymbol{u}^{(m)}(\boldsymbol{x}) \cdot \boldsymbol{R}_{\mathrm{grad}, \mathcal{G}}(\varphi)(\boldsymbol{x}) \mathrm{d} \boldsymbol{x} .
$$

By the definition of the $\|\cdot\|_{-1, \mathcal{D}}$ norm, then by Theorem 4.7, we thus have:

$$
\left|R_{3}\right| \leq c(\varphi) h^{(m)}\left\|\boldsymbol{u}^{(m)}\right\|_{\mathcal{D}^{(m)}} .
$$

As the convergence of $\int_{\Omega} g(\boldsymbol{x})\left(P_{\mathcal{G}^{(m)}} \varphi\right)(\boldsymbol{x}) \mathrm{d} \boldsymbol{x}$ to $\int_{\Omega} g(\boldsymbol{x}) \varphi(\boldsymbol{x}) \mathrm{d} \boldsymbol{x}$ is easily seen, this concludes the proof. 


\subsection{Error analysis}

We prove in this section first order estimates in natural (discrete) energy norms for the discretization error associated to scheme (4.4).

Theorem 4.9 (error estimate). We suppose that hypotheses (2.2)-(2.4) hold. Let $\mathcal{D}$ be a super-admissible discretization of $\Omega$ in the sense of Definitions 3.1 and 3.2 , and $\theta>0$ be such that $\operatorname{regul}(\mathcal{D}, \mathcal{G})>\theta$. Let $\lambda \in(0,+\infty)$ be given. We suppose that the solution to the continuous problem $(2.7),(\overline{\boldsymbol{u}}, \bar{p})$, lies in $\left(\mathrm{H}^{2}(\Omega)^{d} \cap\right.$ $\left.\mathrm{H}_{0}^{1}(\Omega)^{d}\right) \times \mathrm{H}^{1}(\Omega)$. Let $(\boldsymbol{u}, p) \in \mathrm{H}_{\mathcal{D}}(\Omega)^{d} \times \mathrm{H}_{\mathcal{D}}(\Omega)$ be the solution to (4.4). Then there exists two positive real numbers $c_{1}$ and $c_{2}$ depending only on $d, \Omega$ and $\theta$ such that the following two inequalities hold:

$$
\begin{gathered}
\eta\left\|\boldsymbol{u}-P_{\mathcal{D}}(\overline{\boldsymbol{u}})\right\|_{\mathrm{L}^{2}(\Omega)^{d}}^{2}+\nu\left\|\boldsymbol{u}-P_{\mathcal{D}}(\overline{\boldsymbol{u}})\right\|_{\mathcal{D}}^{2}+\frac{c_{1}}{\max [\eta, \nu, 1 / \lambda]}\left\|p-P_{\mathcal{M}}(\bar{p})\right\|_{\mathrm{L}^{2}(\Omega)}^{2} \leq \\
c_{1} h_{\mathcal{M}}^{2}\left[(1+\max [\eta, \nu, 1 / \lambda])\|\overline{\boldsymbol{u}}\|_{\mathrm{H}^{2}(\Omega)^{d}}+\left(\frac{1}{\nu}+\lambda \max [\eta, \nu, 1 / \lambda]\right)|\bar{p}|_{\mathrm{H}^{1}(\Omega)}\right] \\
\eta\left\|\boldsymbol{u}-P_{\mathcal{D}}(\overline{\boldsymbol{u}})\right\|_{\mathrm{L}^{2}(\Omega)^{d}}^{2}+\nu\left\|\boldsymbol{u}-P_{\mathcal{D}}(\overline{\boldsymbol{u}})\right\|_{\mathcal{D}}^{2}+\frac{c_{1}}{\max [\eta, \nu, 1 / \lambda]}\left\|p-P_{\mathcal{G}}(\bar{p})\right\|_{\mathrm{L}^{2}(\Omega)}^{2} \leq \\
c_{2} h_{\mathcal{G}}^{2}\left[(1+\max [\eta, \nu, 1 / \lambda])\|\overline{\boldsymbol{u}}\|_{\mathrm{H}^{2}(\Omega)^{d}}+\frac{1}{\nu}|\bar{p}|_{\mathrm{H}^{1}(\Omega)}\right] .
\end{gathered}
$$

Remark 4.10 (dependence of the error estimate on the stabilization parameter). Note that, in the latter bound, $h_{\mathcal{M}}$ is replaced by $h_{\mathcal{G}}$. In counterpart, this latter estimate is robust with respect to large values of the parameter $\lambda$, in the sense that the right hand side is bounded independently of $\lambda$ when $\lambda \rightarrow+\infty$. This behaviour is natural, since the limiting scheme in this case is obtained by replacing the standard finite-volume approximation of the pressure by the approximation by cluster-wide constant functions, which, associated to the cell-centered approximation for the velocities, has been shown to be inf-sup stable. The resulting flexibility for the choice of $\lambda$ is of great importance in practice.

Proof. Estimate (4.26)

We define $\boldsymbol{e} \in \mathrm{H}_{\mathcal{D}}(\Omega)^{d}$ and $\epsilon \in \mathrm{H}_{\mathcal{D}}(\Omega)$ by $\boldsymbol{e}=\boldsymbol{u}-P_{\mathcal{D}}(\overline{\boldsymbol{u}})$ and $\epsilon=p-P_{\mathcal{M}}(\bar{p})$. Subtracting the same terms from the left and right hand side of the discrete momentum balance equation, we get, for each control volume $K$ of $\mathcal{M}$ :

$$
\begin{aligned}
& \eta m_{K} \boldsymbol{e}_{K}-\nu \sum_{\substack{\sigma \in \mathcal{E}_{\text {int }} \cap \mathcal{E}(K), \sigma=K \mid L}} \frac{m_{\sigma}}{d_{\sigma}}\left(\boldsymbol{e}_{L}-\boldsymbol{e}_{K}\right)-\nu \sum_{\sigma \in \mathcal{E}(K) \cap \mathcal{E}_{\text {ext }}} \frac{m_{\sigma}}{d_{K, \sigma}}\left(-\boldsymbol{e}_{K}\right) \\
& +\sum_{\substack{\sigma \in \mathcal{E}_{\text {int }} \cap \mathcal{E}(K), \sigma=K \mid L}} m_{\sigma}\left[\frac{d_{K, \sigma}}{d_{\sigma}} \epsilon_{K}+\frac{d_{L, \sigma}}{d_{\sigma}} \epsilon_{L}\right] \boldsymbol{n}_{\sigma}+\sum_{\sigma \in \mathcal{E}(K) \cap \mathcal{E}_{\text {ext }}} m_{\sigma} \epsilon_{K} \boldsymbol{n}_{\sigma}=\int_{K} \boldsymbol{f}(\boldsymbol{x}) \mathrm{d} \boldsymbol{x} \\
& -\eta m_{K} \overline{\boldsymbol{u}}\left(\boldsymbol{x}_{K}\right)+\nu \sum_{\substack{\sigma \in \mathcal{E}_{\mathrm{int}} \cap \mathcal{E}(K), \sigma=K \mid L}} \frac{m_{\sigma}}{d_{\sigma}}\left(\overline{\boldsymbol{u}}\left(\boldsymbol{x}_{L}\right)-\overline{\boldsymbol{u}}\left(\boldsymbol{x}_{K}\right)\right)+\nu \sum_{\sigma \in \mathcal{E}(K) \cap \mathcal{E}_{\text {ext }}} \frac{m_{\sigma}}{d_{K, \sigma}}\left(-\overline{\boldsymbol{u}}\left(x_{K}\right)\right) \\
& -\sum_{\substack{\sigma \in \mathcal{E}_{\text {int }} \cap \mathcal{E}(K), \sigma=K \mid L}} m_{\sigma}\left[\frac{d_{K, \sigma}}{d_{\sigma}} P_{\mathcal{M}}(\bar{p})_{K}+\frac{d_{L, \sigma}}{d_{\sigma}} P_{\mathcal{M}}(\bar{p})_{L}\right] \boldsymbol{n}_{\sigma}-\sum_{\sigma \in \mathcal{E}(K) \cap \mathcal{E}_{\text {ext }}} m_{\sigma} P_{\mathcal{M}}(\bar{p})_{K} \boldsymbol{n}_{\sigma} .
\end{aligned}
$$

The regularity of $\overline{\boldsymbol{u}}$ and $\bar{p}$ assumed in the statement of the theorem allows to integrate the continuous partial derivative equation (2.7) over each element $K$ :

$$
0=-\eta \int_{K} \overline{\boldsymbol{u}}(\boldsymbol{x}) \mathrm{d} \boldsymbol{x}+\nu \int_{\partial K} \boldsymbol{\nabla} \overline{\boldsymbol{u}}(\boldsymbol{x}) \cdot \boldsymbol{n} \mathrm{d} \gamma(\boldsymbol{x})-\int_{\partial K} \bar{p}(\boldsymbol{x}) \boldsymbol{n} \mathrm{d} \gamma(\boldsymbol{x})+\int_{K} \boldsymbol{f}(\boldsymbol{x}) \mathrm{d} \boldsymbol{x} .
$$


Subtracting this relation from the previous one, we get, for each control volume $K$ of $\mathcal{M}$ :

$$
\begin{aligned}
\eta m_{K} \boldsymbol{e}_{K}-\nu \sum_{\substack{\sigma \in \mathcal{E}_{\mathrm{int}} \cap \mathcal{E}(K), \sigma=K \mid L}} \frac{m_{\sigma}}{d_{\sigma}}\left(\boldsymbol{e}_{L}-\boldsymbol{e}_{K}\right)-\nu \sum_{\sigma \in \mathcal{E}(K) \cap \mathcal{E}_{\mathrm{ext}}} \frac{m_{\sigma}}{d_{K, \sigma}}\left(-\boldsymbol{e}_{K}\right) \\
+\sum_{\substack{\sigma \in \mathcal{E}_{\mathrm{int}} \cap \mathcal{E}(K), \sigma=K \mid L}} m_{\sigma}\left[\frac{d_{K, \sigma}}{d_{\sigma}} \epsilon_{K}+\frac{d_{L, \sigma}}{d_{\sigma}} \epsilon_{L}\right] \boldsymbol{n}_{\sigma}+\sum_{\sigma \in \mathcal{E}(K) \cap \mathcal{E}_{\mathrm{ext}}} m_{\sigma} \epsilon_{K} \boldsymbol{n}_{\sigma} \\
=-\eta \int_{K} \boldsymbol{R}_{\mathrm{o}}(\overline{\boldsymbol{u}})(\boldsymbol{x}) \mathrm{d} \boldsymbol{x}+\nu \int_{K} \boldsymbol{R}_{\Delta}(\overline{\boldsymbol{u}})(\boldsymbol{x}) \mathrm{d} \boldsymbol{x}-\int_{K} \boldsymbol{R}_{\mathrm{grad}, \mathcal{M}}(\bar{p})(\boldsymbol{x}) \mathrm{d} \boldsymbol{x} .
\end{aligned}
$$

Repeating the same process with the mass balance equation yields, once again for each control volume $K$ of $\mathcal{M}$ :

$$
\begin{aligned}
\sum_{\substack{\sigma \in \mathcal{E}_{\text {int } \cap \mathcal{E}(K),}^{\sigma=K \mid L}, \sigma=}} m_{\sigma}\left(\frac{d_{L, \sigma}}{d_{\sigma}} \boldsymbol{e}_{K}+\frac{d_{K, \sigma}}{d_{\sigma}} \boldsymbol{e}_{L}\right) \cdot \boldsymbol{n}_{\sigma} & -\lambda \sum_{L \in \mathcal{N}_{K} \cap G_{K}}\left(h_{K}+h_{L}\right) m_{\sigma}\left(\epsilon_{L}-\epsilon_{K}\right) \\
& =-\int_{K} R_{\operatorname{div}}(\overline{\boldsymbol{u}})(\boldsymbol{x}) \mathrm{d} \boldsymbol{x}+\lambda \int_{K} R_{\mathrm{stab}, \mathcal{M}}(\bar{p})(\boldsymbol{x}) \mathrm{d} \boldsymbol{x} .
\end{aligned}
$$

The result then follows by combining the estimate of the consistency residuals (Thm. 4.7) with the stability result of Theorem 4.4, with the following choice:

$$
\boldsymbol{f}_{1}=-\eta \boldsymbol{R}_{\circ}(\overline{\boldsymbol{u}}), \quad \boldsymbol{f}_{2}=\nu \boldsymbol{R}_{\Delta}(\overline{\boldsymbol{u}})-\boldsymbol{R}_{\mathrm{grad}, \mathcal{M}}(\bar{p}), \quad g=-R_{\mathrm{div}}(\overline{\boldsymbol{u}})+\lambda R_{\mathrm{stab}, \mathcal{M}}(\bar{p}) .
$$

Estimate (4.27)

The proof of this estimate follows strictly the same line, replacing $P_{\mathcal{M}}(\bar{p})$ by $P_{\mathcal{G}}(\bar{p})$, so that the definition of the pressure error becomes $\epsilon=p-P_{\mathcal{G}}(\bar{p})$. We remark that the stabilization term vanishes when applied to $P_{\mathcal{G}}(\bar{p})$. The system of equations governing the errors is thus formally the same as in the previous case, excepting for the stabilization residual which disappears, and the conclusion once again follows from Theorems 4.4 and 4.7.

\section{The finite volume scheme for the Navier-Stokes Equations}

In this section, we extend the previous results to the Navier-Stokes equations. After presenting the scheme, we prove stability estimates for the solution in natural norms, i.e. the discrete $\mathrm{H}^{1}$ norm and the $\mathrm{L}^{2}$ norm for the velocity and the pressure respectively; by a topological degree argument, these bounds yield the existence of a discrete solution. The remaining of the section is devoted to the convergence analysis.

\subsection{The finite volume scheme}

As in the section devoted to the generalized Stokes problem, we first write the finite volume scheme under consideration in a variational-like setting. Under hypotheses (2.2)-(2.4) and $\mathcal{D}$ being a super-admissible discretization of $\Omega$ in the sense of Definitions 3.1 and 3.2, we look for $(\boldsymbol{u}, p)$ such that:

$$
\mid \begin{array}{ll}
(\boldsymbol{u}, p) \in \mathrm{H}_{\mathcal{D}}(\Omega)^{d} \times \mathrm{H}_{\mathcal{D}}(\Omega) \text { with } \int_{\Omega} p(\boldsymbol{x}) \mathrm{d} \boldsymbol{x}=0, & \\
\eta \int_{\Omega} \boldsymbol{u}(\boldsymbol{x}) \cdot \boldsymbol{v}(\boldsymbol{x}) \mathrm{d} \boldsymbol{x}+\nu[\boldsymbol{u}, \boldsymbol{v}]_{\mathcal{D}}+b_{\mathcal{D}}(\boldsymbol{u}, \boldsymbol{u}, \boldsymbol{v})-\int_{\Omega} p(\boldsymbol{x}) \operatorname{div}_{\mathcal{D}} \boldsymbol{v}(\boldsymbol{x}) \mathrm{d} \boldsymbol{x}=\int_{\Omega} \boldsymbol{f}(\boldsymbol{x}) \cdot \boldsymbol{v}(\boldsymbol{x}) \mathrm{d} \boldsymbol{x} & \forall \boldsymbol{v} \in \mathrm{H}_{\mathcal{D}}(\Omega)^{d} \\
\int_{\Omega} \operatorname{div}_{\mathcal{D}} \boldsymbol{u}(\boldsymbol{x}) q(\boldsymbol{x}) \mathrm{d} \boldsymbol{x}+\lambda\langle p, q\rangle_{\mathcal{G}}=0 & \forall q \in \mathrm{H}_{\mathcal{D}}(\Omega)
\end{array}
$$


where $\lambda$ is a positive real number and, for $\boldsymbol{u}, \boldsymbol{v}, \boldsymbol{w} \in \mathrm{H}_{\mathcal{D}}(\Omega)^{d}$, the trilinear form $b(\boldsymbol{u}, \boldsymbol{v}, \boldsymbol{w})$ is defined by:

$$
b_{\mathcal{D}}(\boldsymbol{u}, \boldsymbol{v}, \boldsymbol{w})=\frac{1}{2} \sum_{K \in \mathcal{M}} \sum_{\substack{\sigma \in \mathcal{E}_{\mathrm{int}} \cap \mathcal{E}(K), \sigma=K \mid L}} m_{\sigma}\left[\frac{d_{L, \sigma} \boldsymbol{u}_{K}+d_{K, \sigma} \boldsymbol{u}_{L}}{d_{\sigma}} \cdot \boldsymbol{n}_{\sigma}\right]\left[\boldsymbol{v}_{L} \cdot \boldsymbol{w}_{K}\right]
$$

Thanks to the definition of the operator $\operatorname{div}_{\mathcal{D}}$ and of the bilinear form $\langle\cdot, \cdot\rangle_{\mathcal{G}}$, we observe that both sides of the second equation of (5.1) vanish for any constant test function $q$; in spite of the assumption $\int_{\Omega} p(\boldsymbol{x}) \mathrm{d} \boldsymbol{x}=0$, system (5.1) is thus equivalent to a square nonlinear system.

Remark 5.1 (on the construction of the trilinear form). Let us introduce the following notation:

$$
G_{\sigma}(\boldsymbol{u})=m_{\sigma} \frac{d_{L, \sigma} \boldsymbol{u}_{K}+d_{K, \sigma} \boldsymbol{u}_{L}}{d_{\sigma}} \cdot \boldsymbol{n}_{\sigma}
$$

The natural discretization of the trilinear form is then:

$$
b_{\mathcal{D}}^{\prime}(\boldsymbol{u}, \boldsymbol{v}, \boldsymbol{w})=\sum_{K \in \mathcal{M}} \sum_{\substack{\sigma \in \mathcal{E}_{\text {int }} \cap \mathcal{E}(K), \sigma=K \mid L}} G_{\sigma}(\boldsymbol{u}) \frac{\boldsymbol{v}_{K}+\boldsymbol{v}_{L}}{2} \cdot \boldsymbol{w}_{K}
$$

Note that this discretization satisfies the antisymmetry property $b_{\mathcal{D}}^{\prime}(\boldsymbol{u}, \boldsymbol{v}, \boldsymbol{w})=-b_{\mathcal{D}}^{\prime}(\boldsymbol{u}, \boldsymbol{w}, \boldsymbol{v})$ for any $\boldsymbol{u}$ satisfying the non-stabilized continuity equation $\int_{\Omega} \operatorname{div}_{\mathcal{D}} \boldsymbol{u}(x) q(x)=0$ for all $q \in \mathrm{H}_{\mathcal{D}}(\Omega)$. An antisymmetrical discretized form $b_{\mathcal{D}}^{\mathrm{a}}(\cdot, \cdot, \cdot)$ can then easily be obtained by defining $b_{\mathcal{D}}^{\mathrm{a}}(\boldsymbol{u}, \boldsymbol{v}, \boldsymbol{w})=1 / 2\left[b_{\mathcal{D}}^{\prime}(\boldsymbol{u}, \boldsymbol{v}, \boldsymbol{w})-b_{\mathcal{D}}^{\prime}(\boldsymbol{u}, \boldsymbol{w}, \boldsymbol{v})\right]$. Computing this latter expression, we get:

$$
b_{\mathcal{D}}^{\mathrm{a}}(\boldsymbol{u}, \boldsymbol{v}, \boldsymbol{w})=\frac{1}{4} \sum_{K \in \mathcal{M}} \sum_{\substack{\sigma \in \mathcal{E}_{\mathrm{int}} \cap \mathcal{E}(K), \sigma=K \mid L}} G_{\sigma}(\boldsymbol{u})\left(\boldsymbol{v}_{L} \cdot \boldsymbol{w}_{K}-\boldsymbol{v}_{K} \cdot \boldsymbol{w}_{L}\right)
$$

By conservativity, we have:

$$
\sum_{K \in \mathcal{M}} \sum_{\substack{\sigma \in \mathcal{E}_{\mathrm{int}} \cap \mathcal{E}(K), \sigma=K \mid L}} G_{\sigma}(\boldsymbol{u})\left(\boldsymbol{v}_{L} \cdot \boldsymbol{w}_{K}+\boldsymbol{v}_{K} \cdot \boldsymbol{w}_{L}\right)=0
$$

We thus obtain that the trilinear form $b_{\mathcal{D}}^{\mathrm{a}}(\cdot, \cdot, \cdot)$ is exactly the same as $b_{\mathcal{D}}(\cdot, \cdot, \cdot)$. Therefore, $b_{\mathcal{D}}(\cdot, \cdot, \cdot)$ is antisymmetrical, which also yields the property (5.3) below. Note that in $[6,14]$, a slightly different discretization of $b_{\mathcal{D}}(\cdot, \cdot, \cdot)$ was introduced, which uses the stabilized mass flux for the discretization of $G_{\sigma}(\boldsymbol{u})$ on the control volume boundaries. This discretization leads to a discrete trilinear form which also depends on the pressure (i.e. $G_{\sigma}(\boldsymbol{u})$ becomes in fact $G_{\sigma}(\boldsymbol{u}, p)$ ) and is also quite efficient (see [6]). However, its analysis in the context of local stabilization studied here is quite technical because of the lack of estimate on the jump of the pressure through the edges separating two clusters.

Using the discrete mass balance to transform the expression of the convection term, system (5.1) is equivalent to finding the family of vectors $\left(\boldsymbol{u}_{K}\right)_{K \in \mathcal{M}} \subset \mathbb{R}^{d}$, and scalars $\left(p_{K}\right)_{K \in \mathcal{M}} \subset \mathbb{R}$ solution to the system of equations 
obtained by writing for each control volume $K$ of $\mathcal{M}$ :

$$
\begin{aligned}
& \mid \begin{array}{l}
\eta m_{K} \boldsymbol{u}_{K}-\nu \sum_{\substack{\sigma \in \mathcal{E}_{\text {int }} \cap \mathcal{E}(K), \sigma=K \mid L}} \frac{m_{\sigma}}{d_{\sigma}}\left(\boldsymbol{u}_{L}-\boldsymbol{u}_{K}\right)-\nu \sum_{\sigma \in \mathcal{E}_{\text {ext }} \cap \mathcal{E}(K)} \frac{m_{\sigma}}{d_{K, \sigma}}\left(0-\boldsymbol{u}_{K}\right) \\
+\sum_{\substack{\sigma \in \mathcal{E}_{\text {int }}^{\sigma=K \mathcal{E}(K), L}, m_{\sigma}}} m_{\sigma}\left[\frac{d_{L, \sigma} \boldsymbol{u}_{K}+d_{K, \sigma} \boldsymbol{u}_{L}}{d_{\sigma}} \cdot \boldsymbol{n}_{\sigma}\right] \frac{\boldsymbol{u}_{K}+\boldsymbol{u}_{L}}{2}-\lambda\left[\sum_{L \in \mathcal{N}_{K} \cap G_{K}} m_{K \mid L}\left(h_{K}+h_{L}\right)\left(p_{L}-p_{K}\right)\right] \frac{\boldsymbol{u}_{K}}{2} \\
+\sum_{\substack{\sigma \in \mathcal{E}_{\text {int }} \cap \mathcal{E}(K), \sigma=K \mid L}} m_{\sigma} \frac{d_{L, \sigma} p_{L}+d_{K, \sigma} p_{K}}{d_{K \mid L}} \boldsymbol{n}_{K \mid L}+\sum_{\sigma \in \mathcal{E}_{\text {ext }} \cap \mathcal{E}(K)} m_{\sigma} p_{K} \boldsymbol{n}_{\sigma}=\int_{K} \boldsymbol{f}(\boldsymbol{x}) \mathrm{d} \boldsymbol{x}
\end{array} \\
& \sum_{\substack{\sigma \in \mathcal{E}_{\text {int } \cap \mathcal{E}(K),}^{\sigma=K \mid L} \\
\sigma=}} \frac{d_{L, \sigma} \boldsymbol{u}_{K}+d_{K, \sigma} \boldsymbol{u}_{L}}{d_{\sigma}} \cdot \boldsymbol{n}_{\sigma}-\lambda \sum_{L \in \mathcal{N}_{K} \cap G_{K}} m_{K \mid L}\left(h_{K}+h_{L}\right)\left(p_{L}-p_{K}\right)=0
\end{aligned}
$$

supplemented by the relation:

$$
\sum_{K \in \mathcal{M}} m_{K} p_{K}=0
$$

The above scheme is written in a conservative form except for the second term in the discretisation of the trilinear form, i.e.:

which stems from the stabilization term.

$$
\lambda\left[\sum_{L \in \mathcal{N}_{K} \cap G_{K}} m_{K \mid L}\left(h_{K}+h_{L}\right)\left(p_{L}-p_{K}\right)\right] \frac{\boldsymbol{u}_{K}}{2}
$$

\subsection{Stability and existence of discrete solutions}

Let us first remark that $b_{\mathcal{D}}(\cdot, \cdot, \cdot)$ is such that, for all $\boldsymbol{u}, \boldsymbol{v} \in \mathrm{H}_{\mathcal{D}}(\Omega)^{d}$ :

$$
b_{\mathcal{D}}(\boldsymbol{u}, \boldsymbol{v}, \boldsymbol{v})=0
$$

Let us also remark that, in a similar way as in [15], Lemma 4.2, we have the existence of $c_{b}$, only depending on $d$ and $\Omega$, such that:

$$
\left|b_{\mathcal{D}}(\boldsymbol{u}, \boldsymbol{v}, \boldsymbol{w})\right| \leq c_{b}\|\boldsymbol{u}\|_{\mathcal{D}}\|\boldsymbol{v}\|_{\mathcal{D}}\|\boldsymbol{w}\|_{\mathcal{D}}, \quad \forall \boldsymbol{u}, \boldsymbol{v}, \boldsymbol{w} \in \mathrm{H}_{\mathcal{D}}(\Omega)^{d}
$$

Lemma 5.2 (estimate for the velocity and the pressure). Under hypotheses (2.2)-(2.4), let $\mathcal{D}$ be an admissible discretization of $\Omega$ in the sense of Definition 3.1 and $\lambda \in(0,+\infty)$ be given. For $\rho \in[0,1]$, we assume that $(\boldsymbol{u}, p)$ is a solution to the following system of equations (which reduces to (5.1) as $\rho=1$ and to (4.4) as $\rho=0)$ :

$$
\begin{array}{|ll}
(\boldsymbol{u}, p) \in \mathrm{H}_{\mathcal{D}}(\Omega)^{d} \times \mathrm{H}_{\mathcal{D}}(\Omega) \text { with } \int_{\Omega} p(\boldsymbol{x}) \mathrm{d} \boldsymbol{x}=0, & \\
\eta \int_{\Omega} \boldsymbol{u}(\boldsymbol{x}) \cdot \boldsymbol{v}(\boldsymbol{x}) \mathrm{d} \boldsymbol{x}+\nu[\boldsymbol{u}, \boldsymbol{v}]_{\mathcal{D}}+\rho b_{\mathcal{D}}(\boldsymbol{u}, \boldsymbol{u}, \boldsymbol{v}) & \\
-\int_{\Omega} p(\boldsymbol{x}) \operatorname{div}_{\mathcal{D}} \boldsymbol{v}(\boldsymbol{x}) \mathrm{d} \boldsymbol{x}=\int_{\Omega} \boldsymbol{f}(\boldsymbol{x}) \cdot \boldsymbol{v}(\boldsymbol{x}) \mathrm{d} \boldsymbol{x} & \forall \boldsymbol{v} \in \mathrm{H}_{\mathcal{D}}(\Omega)^{d} \\
\int_{\Omega} \operatorname{div}_{\mathcal{D}} \boldsymbol{u}(\boldsymbol{x}) q(\boldsymbol{x}) \mathrm{d} \boldsymbol{x}+\lambda\langle p, q\rangle_{\mathcal{G}}=0 & \forall q \in \mathrm{H}_{\mathcal{D}}(\Omega) .
\end{array}
$$


Then $\boldsymbol{u}$ and $p$ satisfy the following estimates:

$$
\nu^{2}\|\boldsymbol{u}\|_{\mathcal{D}}^{2}+2 \nu \lambda|p|_{\mathcal{G}}^{2} \leq \operatorname{diam}(\Omega)^{2}\|\boldsymbol{f}\|_{L^{2}(\Omega)^{d}}^{2} .
$$

Moreover, let $\theta>0$ be such that $\operatorname{regul}(\mathcal{D}, \mathcal{G})>\theta$; then there exist $c_{\mathrm{p}}$ only depending on $d, \Omega, \eta, \nu, \lambda, \boldsymbol{f}, \theta$ and not on $\rho \in[0,1]$ such that the following inequality holds:

$$
\|p\|_{\mathrm{L}^{2}(\Omega)} \leq c_{\mathrm{p}}
$$

Proof. The proof of (5.6) is first obtained by setting $(\boldsymbol{v}, q)=(\boldsymbol{u}, p)$ in (5.5) and using the property (5.3) on the discrete form $b_{\mathcal{D}}(\cdot, \cdot, \cdot)$. Then, by Lemma 4.2 , we know that there exists a function $\boldsymbol{v} \in \mathrm{H}_{\mathcal{D}}(\Omega)^{d}$ such that:

$$
\|\boldsymbol{v}\|_{\mathcal{D}} \leq \beta_{1}\|p\|_{\mathrm{L}^{2}(\Omega)} \quad \text { and } \quad \int_{\Omega} p(\boldsymbol{x}) \operatorname{div}_{\mathcal{D}} \boldsymbol{v}(\boldsymbol{x}) \mathrm{d} \boldsymbol{x} \geq\|p\|_{\mathrm{L}^{2}(\Omega)}^{2}-\beta_{2}|p|_{\mathcal{G}}^{2}
$$

where $\beta_{1}$ and $\beta_{2}$ are two positive real numbers only depending on $d, \Omega$ and $\theta$. Choosing such a function $\boldsymbol{v}$ as test function in the first equation of $(5.5)$, we get:

$$
\|p\|_{\mathrm{L}^{2}(\Omega)}^{2} \leq \beta_{2}|p|_{\mathcal{G}}^{2}+\int_{\Omega} \boldsymbol{f}(\boldsymbol{x}) \cdot \boldsymbol{v}(\boldsymbol{x}) \mathrm{d} \boldsymbol{x}-\eta \int_{\Omega} \boldsymbol{u}(\boldsymbol{x}) \cdot \boldsymbol{v}(\boldsymbol{x}) \mathrm{d} \boldsymbol{x}-\rho b_{\mathcal{D}}(\boldsymbol{u}, \boldsymbol{u}, \boldsymbol{v})-\nu[\boldsymbol{u}, \boldsymbol{v}]_{\mathcal{D}} .
$$

And thus, by the discrete Poincaré inequality and (5.4):

$$
\|p\|_{\mathrm{L}^{2}(\Omega)}^{2} \leq \beta_{2}|p|_{\mathcal{G}}^{2}+\beta_{1}\|p\|_{\mathrm{L}^{2}(\Omega)}\left[\operatorname{diam}(\Omega)\|\boldsymbol{f}\|_{\mathrm{L}^{2}(\Omega)^{d}}+\eta \operatorname{diam}(\Omega)^{2}\|\boldsymbol{u}\|_{\mathcal{D}}+\rho c_{b}\|\boldsymbol{u}\|_{\mathcal{D}}^{2}+\nu\|\boldsymbol{u}\|_{\mathcal{D}}\right]
$$

and the estimate (5.7) follows by bounding $\rho$ by 1 and using (5.6).

We are now in position to prove the existence of at least one solution to scheme (5.1).

Theorem 5.3 (existence of a discrete solution). Under hypotheses (2.2)-(2.4), let $\mathcal{D}$ be an admissible discretization of $\Omega$ in the sense of Definition 3.1, and $\lambda \in(0,+\infty)$ be given. Then there exists at least one $(\boldsymbol{u}, p) \in\left(\mathrm{H}_{\mathcal{D}}(\Omega)\right)^{d} \times \mathrm{H}_{\mathcal{D}}(\Omega)$, solution to $(5.1)$.

Proof. Let us define the vector subspace $V$ of $\mathrm{H}_{\mathcal{D}}(\Omega)^{d} \times \mathrm{H}_{\mathcal{D}}(\Omega)$ by:

$$
V=\left\{(\boldsymbol{u}, p) \in\left(\mathrm{H}_{\mathcal{D}}(\Omega)\right)^{d} \times \mathrm{H}_{\mathcal{D}}(\Omega) \text { such that } \int_{\Omega} p(\boldsymbol{x}) \mathrm{d} \boldsymbol{x}=0\right\} .
$$

Consider the continuous mapping $F: V \times[0,1] \rightarrow V$ such that, for a given $(\boldsymbol{u}, p) \in V$ and $\rho \in[0,1],(\hat{\boldsymbol{u}}, \hat{p})=$ $F(\boldsymbol{u}, p, \rho)$ is defined by:

$$
\begin{array}{|lr}
\int_{\Omega} \hat{\boldsymbol{u}}(\boldsymbol{x}) \cdot \boldsymbol{v}(\boldsymbol{x}) \mathrm{d} \boldsymbol{x}=\eta \int_{\Omega} \boldsymbol{u}(\boldsymbol{x}) \cdot \boldsymbol{v}(\boldsymbol{x}) \mathrm{d} \boldsymbol{x}+\nu[\boldsymbol{u}, \boldsymbol{v}]_{\mathcal{D}}+\rho b_{\mathcal{D}}(\boldsymbol{u}, \boldsymbol{u}, \boldsymbol{v}) & \\
-\int_{\Omega} p(\boldsymbol{x}) \operatorname{div}_{\mathcal{D}} \boldsymbol{v}(\boldsymbol{x}) \mathrm{d} \boldsymbol{x}-\int_{\Omega} \boldsymbol{f}(\boldsymbol{x}) \cdot \boldsymbol{v}(\boldsymbol{x}) \mathrm{d} \boldsymbol{x} & \forall \boldsymbol{v} \in \mathrm{H}_{\mathcal{D}}(\Omega)^{d} \\
\int_{\Omega} \hat{p}(\boldsymbol{x}) \cdot q(\boldsymbol{x}) \mathrm{d} \boldsymbol{x}=\int_{\Omega} \operatorname{div}_{\mathcal{D}} \boldsymbol{u}(\boldsymbol{x}) q(\boldsymbol{x}) \mathrm{d} \boldsymbol{x}+\lambda\langle p, q\rangle_{\mathcal{G}} & \forall q \in \mathrm{H}_{\mathcal{D}}(\Omega) .
\end{array}
$$

It is easily checked that the two above relations define a one to one function $F(., .,$.$) . Indeed, the value of \hat{\boldsymbol{u}}_{K}^{(i)}$ and $\hat{p}_{K}$ for a given $K \in \mathcal{M}$ and $i=1, \ldots, d$ are readily obtained by setting in this system $\boldsymbol{v}^{(i)}=1_{K}, \boldsymbol{v}^{(j)}=0$ for $j \neq i$, and $q=1_{K}$. 
The mapping $F(., . .$.$) is continuous, and, for a given (\boldsymbol{u}, p)$ such that $F(\boldsymbol{u}, p, \rho)=(0,0)$, we can apply Lemma 5.2, which proves that $(\boldsymbol{u}, p)$ is bounded independently of $\rho$. Since $F(\boldsymbol{u}, p, 0)$ is a bijective affine function of $(\boldsymbol{u}, p)$ from $V$ to $V$ (by Cor. 4.6), the existence of at least one solution $(\boldsymbol{u}, p)$ to the equation $F(\boldsymbol{u}, p, 1)=(0,0)$, which is exactly (5.1), follows by a topological degree argument (see [8] for the theory and [15], Thm. 4.3, for a precise formulation of the abstract theorem used here).

\subsection{Convergence analysis}

We first begin by a technical lemma.

Lemma 5.4. Under hypothesis (2.2), let $\theta>0$ be given and let $\left(\mathcal{D}^{(m)}\right)_{m \in \mathbb{N}}$ be a sequence of admissible discretizations of $\Omega$ in the sense of Definition 3.1 , such that $\lim _{m \rightarrow+\infty} h_{\mathcal{D}}^{(m)}=0$ and such that $\operatorname{regul}\left(\mathcal{D}^{(m)}\right) \geq \theta$, for all $m \in \mathbb{N}$. Let $\left(u^{(m)}\right)_{m \in \mathbb{N}}$ be a sequence satisfying the following assumptions: $\forall m \in \mathbb{N}, u^{(m)} \in \mathrm{H}_{\mathcal{D}^{(m)}}(\Omega)$, there exists $\bar{u} \in \mathrm{H}_{0}^{1}(\Omega)$ such that $u^{(m)}$ converges to $\bar{u}$ in $\mathrm{L}^{2}(\Omega)$ as $m$ tends to $+\infty$ and $\left\|u^{(m)}\right\|_{\mathcal{D}}$ remains bounded.

(1) For each edge $\sigma$ of the mesh and $m \in \mathbb{N}$, we define:

$$
\begin{array}{ll}
\text { if } \sigma \in \mathcal{E}_{\mathrm{int}}, \sigma=K \mid L & \left(\overline{\boldsymbol{\nabla}}_{\mathcal{D}} u^{(m)}\right)_{\sigma}=d \frac{1}{d_{\sigma}}\left[u_{L}^{(m)}-u_{K}^{(m)}\right] \boldsymbol{n}_{\sigma}, \\
\text { if } \sigma \in \mathcal{E}_{\mathrm{ext}}, \sigma \in \mathcal{E}(K) & \left(\overline{\boldsymbol{\nabla}}_{\mathcal{D}} u^{(m)}\right)_{\sigma}=d \frac{1}{d_{\sigma}}\left[0-u_{K}^{(m)}\right] \boldsymbol{n}_{\sigma},
\end{array}
$$

and we denote by $\overline{\boldsymbol{\nabla}}_{\mathcal{D}} u^{(m)}$ the piecewise constant function equal to $\left(\overline{\boldsymbol{\nabla}}_{\mathcal{D}} u^{(m)}\right)_{\sigma}$ on the diamond cell $D_{\sigma}$ associated to $\sigma$.

Then the sequence $\overline{\boldsymbol{\nabla}}_{\mathcal{D}} u^{(m)}$ weakly converges to $\boldsymbol{\nabla} \bar{u}$ in $\mathrm{L}^{2}(\Omega)^{d}$.

(2) For each edge $\sigma$ of the mesh and $m \in \mathbb{N}$, we define:

$$
\begin{array}{ll}
\text { if } \sigma \in \mathcal{E}_{\text {int }}, \sigma=K \mid L & u_{\sigma}^{(m)}=\frac{d_{L, \sigma}}{d_{\sigma}} u_{K}^{(m)}+\frac{d_{K, \sigma}}{d_{\sigma}} u_{L}^{(m)}, \\
\text { if } \sigma \in \mathcal{E}_{\text {ext }}, & u_{\sigma}^{(m)}=0,
\end{array}
$$

and we denote by $I_{\mathcal{E}}^{(m)} u^{(m)}$ the piecewise constant function equal to $u_{\sigma}^{(m)}$ on the diamond cell $D_{\sigma}$ associated to $\sigma$.

Then the sequence $I_{\mathcal{E}}^{(m)} u^{(m)}$ tends to $\bar{u}$ in $\mathrm{L}^{p}(\Omega)$, where $2 \leq p<+\infty$ if $d=2$ and $2 \leq p<6$ if $d=3$.

Proof. The proof of item (1) is given in [9], Lemma 2. For the proof of item (2), we first remark that, thanks to the discrete Sobolev inequalities $\|u\|_{L^{r}(\Omega)} \leq c\|u\|_{\mathcal{D}^{(m)}}$ for $2 \leq r<+\infty$ if $d=2$ and for $2 \leq r \leq 6$ if $d=3$ (see [7] or [11], p. 790). We thus have:

$$
\begin{aligned}
\left\|I_{\mathcal{E}}^{(m)} u^{(m)}\right\|_{L^{r}(\Omega)}^{r} & =\sum_{\substack{\sigma \in \mathcal{E}_{\text {int }}, \sigma=K \mid L}} m_{D_{\sigma}}\left|\frac{d_{L, \sigma}}{d_{\sigma}} u_{K}^{(m)}+\frac{d_{K, \sigma}}{d_{\sigma}} u_{L}^{(m)}\right|^{r} \leq 2^{r} \sum_{\substack{\sigma \in \mathcal{E}_{\text {int }}, \sigma=K \mid L}} m_{D_{\sigma}}\left[\left|u_{K}^{(m)}\right|^{r}+\left|u_{L}^{(m)}\right|^{r}\right] \\
& =2^{r} \sum_{K \in \mathcal{M}}\left[\sum_{\sigma \in \mathcal{E}(K)} m_{D_{\sigma}}\right]\left|u_{K}^{(m)}\right|^{r} \leq c\left\|u^{(m)}\right\|_{L^{r}(\Omega)}^{r},
\end{aligned}
$$


where $c \in \mathbb{R}_{+}$only depends on the parameter $\theta$ and $m_{D_{\sigma}}$ denotes the measure of the subset $D_{\sigma}$; the sequence $\left(I_{\mathcal{E}}^{(m)} u^{(m)}\right)_{m \in \mathbb{N}}$ is therefore bounded in $\mathrm{L}^{r}(\Omega)$. On the other hand, we also have:

$$
\left\|u^{(m)}-I_{\mathcal{E}}^{(m)} u^{(m)}\right\|_{\mathrm{L}^{2}(\Omega)}^{2} \leq \sum_{\substack{\sigma \in \mathcal{E}_{\text {int }}, \sigma=K \mid L}} m_{D_{\sigma}}\left(u_{K}^{(m)}-u_{L}^{(m)}\right)^{2} \leq c h_{\mathcal{D}^{(m)}}^{2}\left\|u^{(m)}\right\|_{\mathcal{D}^{(m)}}^{2}
$$

and so the sequence $\left(I_{\mathcal{E}}^{(m)} u^{(m)}\right)_{m \in \mathbb{N}}$ tends to $\bar{u}$ in $\mathrm{L}^{2}(\Omega)$, which implies the result.

We can now state the convergence result for the scheme (5.1).

Theorem 5.5 (convergence of the scheme). Under hypotheses $(2.2)-(2.4)$, let $\left(\mathcal{D}^{(m)}\right)_{m \in \mathbb{N}}$ be a sequence of superadmissible discretizations of $\Omega$ in the sense of Definitions 3.1 and 3.2 , such that $h_{\mathcal{D}}^{(m)}$ tends to 0 as $m \rightarrow+\infty$ and such that there exists $\theta>0$ with $\operatorname{regul}\left(\mathcal{D}^{(m)}, \mathcal{G}^{(m)}\right) \geq \theta$, for all $m \in \mathbb{N}$. Let $\lambda \in(0,+\infty)$ be given. Let, for all $m \in \mathbb{N},\left(\boldsymbol{u}^{(m)}, p^{(m)}\right) \in \mathrm{H}_{\mathcal{D}^{(m)}}(\Omega)^{d} \times \mathrm{H}_{\mathcal{D}^{(m)}}(\Omega)$, be a solution to $(5.1)$ with $\mathcal{D}=\mathcal{D}^{(m)}$. Then there exists a weak solution $(\overline{\boldsymbol{u}}, \bar{p})$ to $(2.5)$ and a subsequence of $\left(\mathcal{D}^{(m)}\right)_{m \in \mathbb{N}}$, again denoted $\left(\mathcal{D}^{(m)}\right)_{m \in \mathbb{N}}$, such that the corresponding subsequence $\left(\boldsymbol{u}^{(m)}\right)_{m \in \mathbb{N}}$ converges to $\overline{\boldsymbol{u}}$ in $\mathrm{L}^{2}(\Omega)^{d}$ and the subsequence $\left(p^{(m)}\right)_{m \in \mathbb{N}}$ converges to $\bar{p}$ weakly in $\mathrm{L}^{2}(\Omega)$.

Proof. Thanks to the fact that the sequence $\left\|\boldsymbol{u}^{(m)}\right\|_{\mathcal{D}^{(m)}}$ is bounded independently of $m$ (estimate (5.6)), we obtain the existence of a subsequence of $\left(\mathcal{D}^{(m)}\right)_{m \in \mathbb{N}}$ and of $\overline{\boldsymbol{u}} \in \mathrm{H}_{0}^{1}(\Omega)^{d}$ such that $\left(\boldsymbol{u}^{(m)}\right)_{m \in \mathbb{N}}$ converges to $\overline{\boldsymbol{u}}$ in $\mathrm{L}^{2}(\Omega)^{d}$. We then again denote $\left(\mathcal{D}^{(m)}\right)_{m \in \mathbb{N}}$ this subsequence. In addition, thanks to the fact that $\left\|p^{(m)}\right\|_{\mathrm{L}^{2}(\Omega)}$ is bounded independently of $m$ by the estimate (5.7), we extract from this subsequence another one (still denoted $\left.\left(\mathcal{D}^{(m)}\right)_{m \in \mathbb{N}}\right)$ such that $\left(p^{(m)}\right)_{m \in \mathbb{N}}$ weakly converges to some function $\bar{p}$ in $\mathrm{L}^{2}(\Omega)$.

With exactly the same arguments as for the Stokes problem, we get $\operatorname{div} \overline{\boldsymbol{u}}(x)=0$ for a.e. $x$ in $\Omega$.

In order to prove the convergence of the scheme, we now must show that $(\overline{\boldsymbol{u}}, \bar{p})$ is a solution to the first equation of the continuous problem (2.5). By density, it is sufficient to prove that this variational problem is satisfied for any test function in $C_{c}^{\infty}(\Omega)^{d}$. As for the Stokes problem, this will be proved by passing to the limit in the scheme. We thus take $\varphi \in C_{c}^{\infty}(\Omega)^{d}$, and suppose that $m$ is large enough and thus $h_{\mathcal{D}}^{(m)}$ is small enough to ensure that, for all $K \in \mathcal{M}$ such that $K \cap \operatorname{support}(\varphi) \neq \emptyset, \partial K \cap \partial \Omega=\emptyset$. Let us take $\boldsymbol{v}=P_{\mathcal{D}^{(m)}} \varphi$ in $(4.4)$, which yields:

$$
\begin{array}{r}
\eta \int_{\Omega} \boldsymbol{u}^{(m)}(\boldsymbol{x}) \cdot P_{\mathcal{D}^{(m)}} \boldsymbol{\varphi}(\boldsymbol{x}) \mathrm{d} \boldsymbol{x}+\nu\left[\boldsymbol{u}^{(m)}, P_{\mathcal{D}^{(m)}} \boldsymbol{\varphi}\right]_{\mathcal{D}^{(m)}}-\int_{\Omega} p^{(m)}(\boldsymbol{x}) \operatorname{div}_{\mathcal{D}^{(m)}}\left(P_{\mathcal{D}^{(m)}} \boldsymbol{\varphi}\right)(\boldsymbol{x}) \mathrm{d} \boldsymbol{x} \\
+b_{\mathcal{D}^{(m)}}\left(\boldsymbol{u}^{(m)}, \boldsymbol{u}^{(m)}, P_{\mathcal{D}^{(m)}} \boldsymbol{\varphi}\right)=\int_{\Omega} \boldsymbol{f}(\boldsymbol{x}) \cdot P_{\mathcal{D}^{(m)}} \boldsymbol{\varphi}(\boldsymbol{x}) \mathrm{d} \boldsymbol{x} .
\end{array}
$$

The convergence of the first three terms at the left hand side of this relation, together with the convergence of the right hand side has been dealt with in the convergence proof for the Stokes problem. To conclude the present convergence analysis, it is thus sufficient to prove that:

$$
\lim _{m \rightarrow+\infty} b_{\mathcal{D}^{(m)}}\left(\boldsymbol{u}^{(m)}, \boldsymbol{u}^{(m)}, P_{\mathcal{D}^{(m)}} \boldsymbol{\varphi}\right)=b(\overline{\boldsymbol{u}}, \overline{\boldsymbol{u}}, \boldsymbol{\varphi})
$$

We remark that, for any two neighbouring control volumes $K$ and $L$ :

$$
\boldsymbol{u}_{L} \cdot \boldsymbol{v}_{K}-\boldsymbol{u}_{K} \cdot \boldsymbol{v}_{L}=\left(\boldsymbol{u}_{L}-\boldsymbol{u}_{K}\right) \cdot \boldsymbol{v}_{K \mid L}+\left(\boldsymbol{v}_{K}-\boldsymbol{v}_{L}\right) \cdot \boldsymbol{u}_{K \mid L}
$$

where, for any function of $\mathrm{H}_{\mathcal{D}^{(m)}}(\Omega)^{d}$, we introduce the following notation:

$$
\forall \sigma \in \mathcal{E}_{\text {int }}^{(m)}, \sigma=K \mid L, \quad \boldsymbol{v}_{\sigma}=\frac{d_{L, \sigma} \boldsymbol{v}_{K}+d_{K, \sigma} \boldsymbol{v}_{L}}{d_{K \mid L}} .
$$


The quantity $b_{\mathcal{D}}\left(\boldsymbol{u}^{(m)}, \boldsymbol{u}^{(m)}, P_{\mathcal{D}^{(m)}} \boldsymbol{\varphi}\right)$ then reads:

$$
\begin{aligned}
& b_{\mathcal{D}}\left(\boldsymbol{u}^{(m)}, \boldsymbol{u}^{(m)}, P_{\mathcal{D}^{(m)}} \boldsymbol{\varphi}\right)=\frac{1}{2} \sum_{i=1}^{d} \sum_{K \in \mathcal{M}} \sum_{\substack{\sigma \in \mathcal{E}_{\operatorname{int} \cap \mathcal{E}(K),} \sigma=K \mid L \\
\sigma=}} m_{\sigma}\left[\boldsymbol{u}_{\sigma}^{(m)} \cdot \boldsymbol{n}_{\sigma}\right]\left[\boldsymbol{u}_{L}^{(m, i)} \boldsymbol{\varphi}^{(i)}\left(\boldsymbol{x}_{K}\right)\right] \\
& =\frac{1}{2} \sum_{i=1}^{d} \sum_{\sigma \in \mathcal{E}_{\text {int }}, \sigma=K \mid L} m_{\sigma}\left[\boldsymbol{u}_{\sigma}^{(m)} \cdot \boldsymbol{n}_{\sigma}\right]\left[\boldsymbol{u}_{L}^{(m, i)} \boldsymbol{\varphi}^{(i)}\left(\boldsymbol{x}_{K}\right)-\boldsymbol{u}_{K}^{(m, i)} \boldsymbol{\varphi}^{(i)}\left(\boldsymbol{x}_{L}\right)\right] \\
& =\frac{1}{2} \sum_{i=1}^{d} \underbrace{\sum_{\sigma \in \mathcal{E}_{\mathrm{int}}, \sigma=K \mid L} m_{\sigma}\left[\boldsymbol{u}_{\sigma}^{(m)} \cdot \boldsymbol{n}_{\sigma}\right]\left[\boldsymbol{u}_{L}^{(m, i)}-\boldsymbol{u}_{K}^{(m, i)}\right]\left(P_{\mathcal{D}^{(m)}} \boldsymbol{\varphi}^{(i)}\right)_{\sigma}}_{\boldsymbol{T}_{1}^{(m, i)}} \\
& +\frac{1}{2} \sum_{i=1}^{d} \underbrace{\sum_{\sigma \in \mathcal{E}_{\text {int }}, \sigma=K \mid L} m_{\sigma}\left[\boldsymbol{u}_{\sigma}^{(m)} \cdot \boldsymbol{n}_{\sigma}\right]\left[\boldsymbol{\varphi}^{(i)}\left(\boldsymbol{x}_{K}\right)-\boldsymbol{\varphi}^{(i)}\left(\boldsymbol{x}_{L}\right)\right] \boldsymbol{u}_{\sigma}^{(m, i)}}_{\boldsymbol{T}_{2}^{(m, i)}} .
\end{aligned}
$$

The term $\boldsymbol{T}_{1}^{(m, i)}$ equivalently reads:

$$
\begin{aligned}
\boldsymbol{T}_{1}^{(m, i)} & =\sum_{\sigma \in \mathcal{E}_{\mathrm{int}}, \sigma=K \mid L} m_{D_{\sigma}}\left(P_{\mathcal{D}^{(m)}} \boldsymbol{\varphi}^{(i)}\right)_{\sigma} \boldsymbol{u}_{\sigma}^{(m)} \cdot\left[d \frac{\boldsymbol{u}_{L}^{(m, i)}-\boldsymbol{u}_{K}^{(m, i)}}{d_{\sigma}} \boldsymbol{n}_{\sigma}\right] \\
& =\int_{\Omega} I_{\mathcal{E}}^{(m)} P_{\mathcal{D}^{(m)}} \boldsymbol{\varphi}^{(i)}(\boldsymbol{x}) I_{\mathcal{E}}^{(m)} \boldsymbol{u}^{(m)}(\boldsymbol{x}) \cdot \bar{\nabla}_{\mathcal{D}^{(m)}} \boldsymbol{u}^{(m, i)}(\boldsymbol{x}) \mathrm{d} \boldsymbol{x}
\end{aligned}
$$

where, for the terms $I_{\mathcal{E}}^{(m)} P_{\mathcal{D}^{(m)}} \varphi^{(i)}$ and $I_{\mathcal{E}}^{(m)} \boldsymbol{u}^{(m)}$, we use the notation introduced in Lemma 5.4. The sequence $\left(P_{\mathcal{D}^{(m)}} \varphi^{(i)}\right)_{m \in \mathbb{N}}$ can be seen to converge to $\varphi^{(i)}$ in $\mathrm{L}^{2}(\Omega)$ and to be bounded in $\mathrm{H}_{\mathcal{D}^{(m)}}(\Omega)$, thus, by the same Lemma 5.4, the sequence $\left(I_{\mathcal{E}}^{(m)} P_{\mathcal{D}^{(m)}} \varphi^{(i)}\right)_{m \in \mathbb{N}}$ converges to $\varphi^{(i)}$ in $\mathrm{L}^{4}(\Omega)$. The same holds for the sequence $\left(I_{\mathcal{E}}^{(m)} \boldsymbol{u}^{(m)}\right)_{m \in \mathbb{N}}$. Finally, still by Lemma $5.4,\left(\overline{\boldsymbol{\nabla}}_{\mathcal{D}(m)} \boldsymbol{u}^{(m, i)}\right)_{m \in \mathbb{N}}$ weakly converges to $\boldsymbol{\nabla} \overline{\boldsymbol{u}}^{(i)}$ in $\mathrm{L}^{2}(\Omega)^{d}$. We thus can pass to the limit in the above equation to obtain:

$$
\lim _{m \rightarrow+\infty} \boldsymbol{T}_{1}^{(m, i)}=\int_{\Omega} \boldsymbol{\varphi}^{(i)}(\boldsymbol{x}) \overline{\boldsymbol{u}}(\boldsymbol{x}) \cdot \boldsymbol{\nabla} \overline{\boldsymbol{u}}^{(i)}(\boldsymbol{x}) \mathrm{d} \boldsymbol{x} .
$$

By the same arguments, we get:

$$
\lim _{m \rightarrow+\infty} \boldsymbol{T}_{2}^{(m, i)}=-\int_{\Omega} \overline{\boldsymbol{u}}^{(i)}(\boldsymbol{x}) \overline{\boldsymbol{u}}(\boldsymbol{x}) \cdot \boldsymbol{\nabla} \boldsymbol{\varphi}^{(i)}(\boldsymbol{x}) \mathrm{d} \boldsymbol{x} .
$$

Since $\operatorname{div} \overline{\boldsymbol{u}}=0$, both limits are equal and the proof of convergence is complete.

\section{NumERICAL TESTS}

The aim of this section is to check the validity of the theoretical analysis against a practical test case for which an analytical solution can be exhibited. This solution is built as follows. We choose a streamfunction 


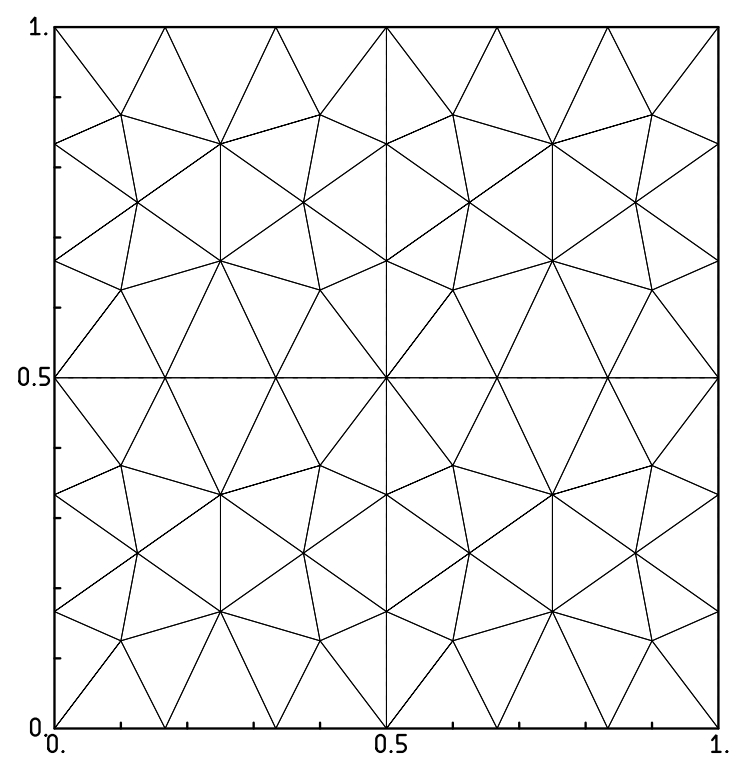

Figure 3. Coarsest mesh of the computational domain in clusters.

and a geometrical domain such that homogeneous Dirichlet conditions hold:

$$
\left.\varphi=1000[x(1-x) y(1-y)]^{2}, \quad \Omega=\right] 0,1[\times] 0,1\left[, \quad \overline{\boldsymbol{u}}=\left[\begin{array}{c}
\frac{\partial \varphi}{\partial y} \\
-\frac{\partial \varphi}{\partial x}
\end{array}\right]\right.
$$

we pick an arbitrary (zero mean value) pressure in $\mathrm{L}^{2}(\Omega)$ :

$$
\bar{p}=100\left(x^{2}+y^{2}-\frac{2}{3}\right)
$$

and the right hand side $f$ is computed so $\bar{u}$ and $\bar{p}$ are solutions to the stationary Navier-Stokes equations, written in dimensional form:

$$
\rho(\overline{\boldsymbol{u}} \boldsymbol{\nabla}) \overline{\boldsymbol{u}}-\mu \Delta \overline{\boldsymbol{u}}+\nabla \bar{p}=\boldsymbol{f} .
$$

To obtain the numerical results displayed here, the practical implementation has been performed using the software object-oriented component library PELICANS, developed at IRSN [22].

The velocity and pressure errors are defined respectively as:

$$
\boldsymbol{e}_{K}^{(i)}=\boldsymbol{u}_{K}^{(i)}-\overline{\boldsymbol{u}}^{(i)}\left(\boldsymbol{x}_{K}\right), \quad \epsilon_{K}=p_{K}-\bar{p}\left(\boldsymbol{x}_{K}\right) .
$$

This pressure error definition is not the same as in the analysis; however it is easy to see from Theorem 4.7 that, for a regular pressure field (for instance, in $\mathrm{H}^{2}(\Omega)$ ), this definition equivalently leads to a first order convergence.

The partition of the computational domain in clusters is built by first splitting the domain into sub-squares and then cutting each sub-square into 26 triangles, all having angles of at most $80^{\circ}$ (corresponding to Fig. 5 


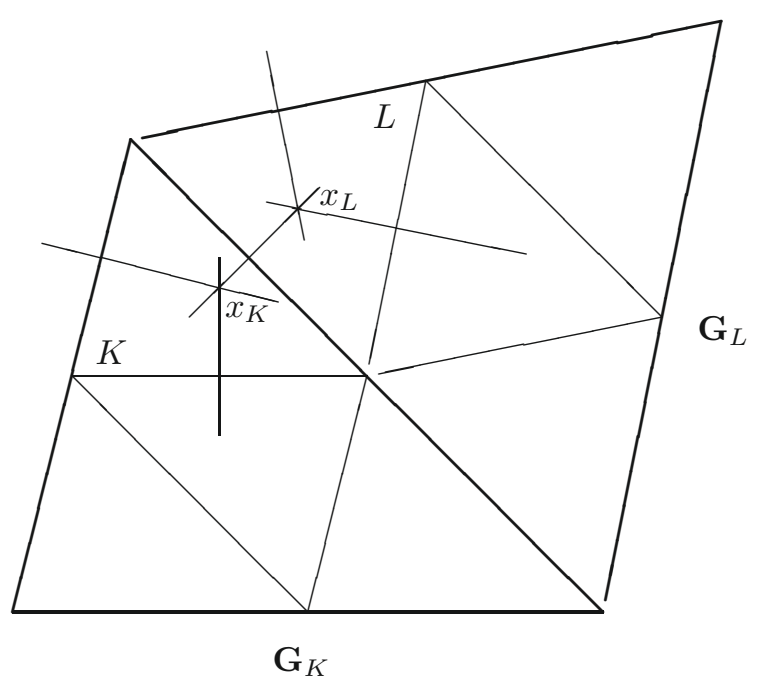

FIGURE 4. Example of clustered simplicial mesh, as used in numerical experiments.

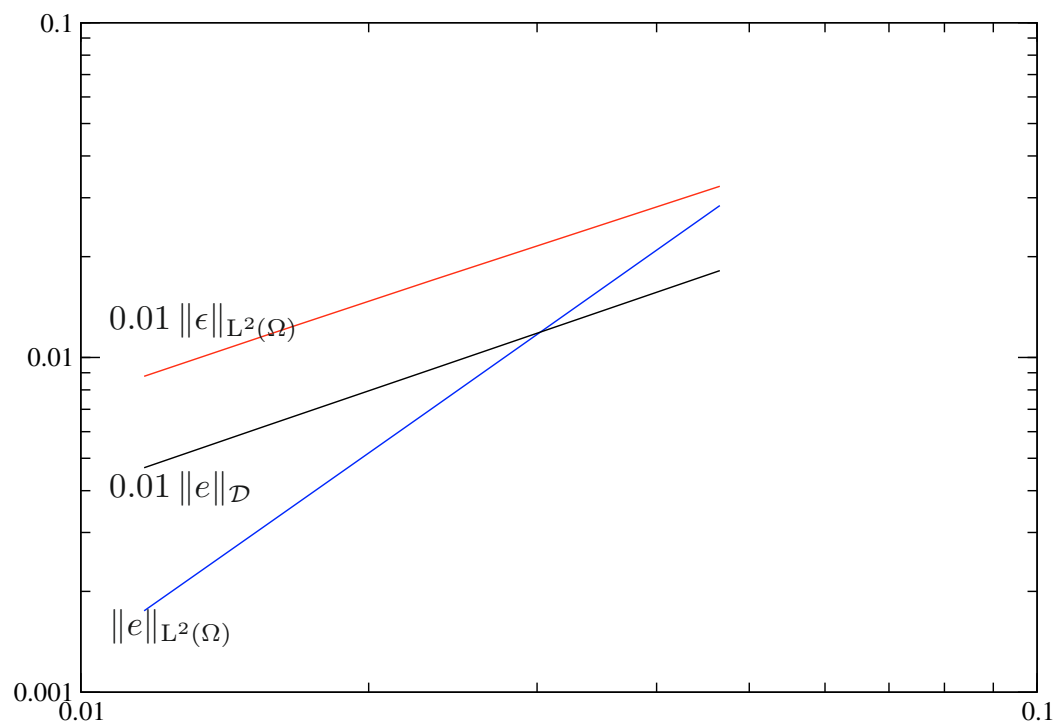

Figure 5. Errors for the velocity and the pressure obtained for the Stokes problem, as a function of the mesh parameter $h_{\mathcal{D}}$.

- bbbb in [2]). The coarsest one is displayed in Figure 3. Control volumes are then obtained by cutting each cluster into four similar triangles, the vertices of which are located on the mid-points of each edge, as shown in Figure 4.

We first begin by the Stokes problem, obtained by setting the density $\rho$ to zero and the viscosity $\mu$ to 1 . The norms of the errors between the numerical and the exact solution are displayed in Figure 5; we observe a first 


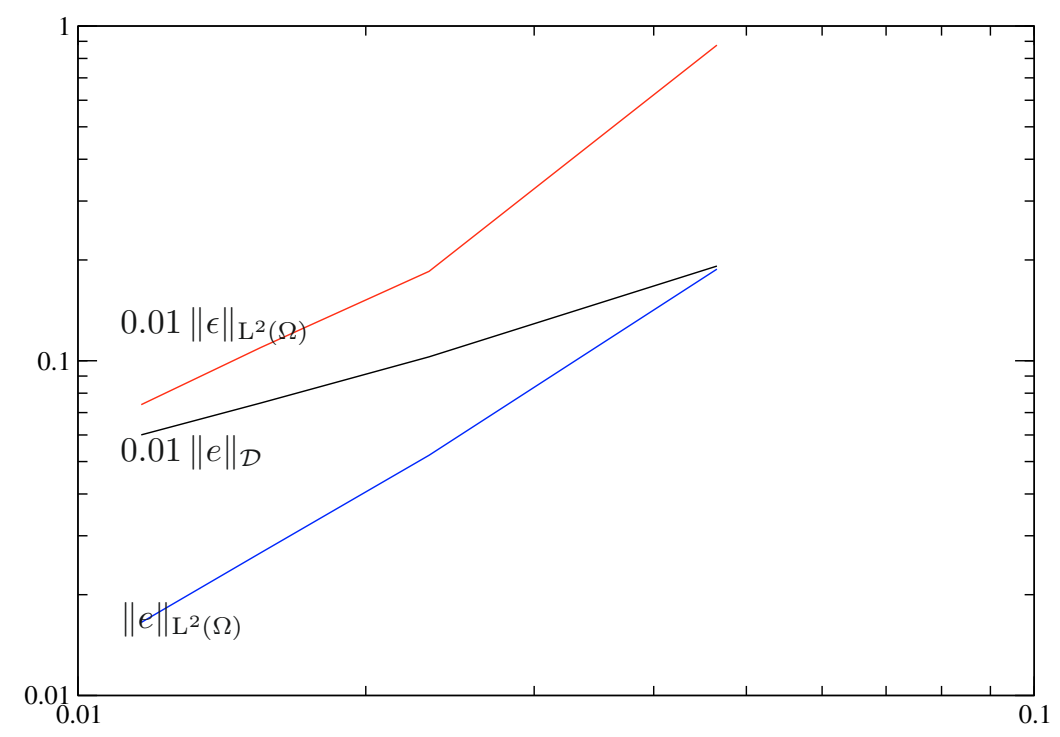

FiguRE 6. Errors for the velocity and the pressure obtained in the Navier-Stokes case $(\mathrm{Re} \approx 1000)$, as a function of the mesh parameter $h_{\mathcal{D}}$.

order convergence for the velocity and the pressure in respectively discrete $\mathrm{H}^{1}$ and $\mathrm{L}^{2}$ norms, and a second order convergence for the velocity in the discrete $\mathrm{L}^{2}$ norm.

We then turn to Navier-Stokes equations, setting $\rho=100$ and $\mu=1$ which, in view of the value of the velocity, leads to a Reynolds number $R e$ in the range of $R e \approx 1000$. We observe (Fig. 6) a noticeable loss of accuracy for the pressure, the convergence of which is, as a counterpart, faster than 1 at high value of the mesh size $h_{\mathcal{D}}$. For coarse grids, pressure oscillations are observed, which do not affect the velocity field; these pressure oscillations do not appear for the Stokes problem, neither for the Navier-Stokes equations when using a mesh based on rectangles (to construct such meshes, clusters are built first by a structured gridding of the domain, then control volumes are obtained by cutting each (rectangular) cluster in four, along the lines joining the mid-edge points). Once again, a first and second order convergence is obtained for the velocity in respectively discrete $\mathrm{H}^{1}$ and $\mathrm{L}^{2}$ norms.

Finally, numerical experiments show that the accuracy of the results is almost insensitive to the stabilization parameter $\lambda$, provided that $\lambda \geq 0.1$ : indeed, for the studied Navier-Stokes case and an intermediate mesh $\left(h_{\mathcal{D}} \approx 0.02\right.$ ), only a difference of less than $20 \%$ on the magnitude of the errors is obtained when varying $\lambda$ up to 10 . For lower values of $\lambda$, the accuracy of the pressure first deteriorates, then the velocity is affected; for $\lambda=0.001$, the error is multiplied by 2 for the velocity and by 7 for the pressure. These results are quite different when using a stabilization à la Brezzi-Pitkäranta, as previously proposed [13,15]: for low values of $\lambda$, results are equivalent with the present scheme but errors dramatically increase when $\lambda$ reaches unity. This behaviour was already evidenced in [6], where the calculated reattachment length of the flow beyond a backward facing step was shown to be very sensitive to the stabilization parameter with the Brezzi-Pitkäranta variant and quite unsensitive with the clustered scheme.

Further tests of the scheme analysed in this paper can be found in $[5,6]$. They include comparisons to analytical solutions, for the Stokes problem, for Navier-Stokes equations (the classical Green-Taylor vortex test) and for an incompressible anisotherm case, together with classical benchmarks, as the two and three dimensional lid driven cavity or the backward facing step, up to more "industrial" natural convection flows. 
In these works (including the present one), the discrete nonlinear system is solved with a Newton method, sometimes with strong relaxation; at each iteration, the linear system is solved by a direct or a Krylov subspace method. An augmented Lagrangian technique also has been implemented. However, this practical issue should clearly deserve further work.

\section{Conclusion}

We presented and analysed in this paper a novel cell-centered collocated finite volume scheme for incompressible flow problems. This scheme is shown to be stable and convergent for the Navier-Stokes equations; moreover, we prove that it is first-order accurate in natural energy norms for the Stokes problem. Numerical experiments confirm the analysis and show, in addition, that the scheme is still first order accurate for a high Reynolds number problem; in addition, a second order convergence for the velocity in a discrete $L^{2}$ norm is observed in any case. Unfortunately, these properties only hold for a particular class of meshes, the so-called super-admissible meshes, which is rather restrictive in practice; getting rid of these limitations is clearly a topic of interest for future work, and results in this direction have already be obtained [10].

One underlying argument of this analysis is that the pair of discrete spaces associating the classical cellcentered approximation for the velocities and cluster-wide constant pressures is inf-sup stable; to our knowledge, this is the first result of this type for finite volume collocated discretizations.

The present work is already extended in practical applications to unsteady problems, also involving heat transfer, either within the framework of the Boussinesq approximation or using the more general asymptotic model for low Mach-number flows [5,6]. These problems should deserve more attention in the future, both from a theoretical point of view as for the design of efficient numerical solvers.

\section{A. Appendix: Proof of the consistency Results}

We begin this section by stating a trace lemma which will be used in the following developments.

Lemma A.1 (a trace inequality). Let $\sigma$ be a bounded measurable subset of an hyperplane of $\mathbb{R}^{3}$, $\boldsymbol{x}_{\mathrm{c}}$ a point of $\mathbb{R}^{3}$ located outside $\sigma$. We denote by $d_{\boldsymbol{x}_{\mathrm{c}}, \sigma}$ the distance from $\boldsymbol{x}_{\mathrm{c}}$ to $\sigma$ and by $D_{\sigma, 1 / 2}$ the volume defined by:

$$
D_{\sigma, 1 / 2}=\left\{t \boldsymbol{x}+(1-t) \boldsymbol{x}_{\mathrm{c}}, \boldsymbol{x} \in \sigma, t \in\left(\frac{1}{2}, 1\right)\right\} .
$$

Let $v$ be a function of $\mathrm{H}^{1}\left(D_{\sigma, 1 / 2}\right)$. Then the following bound holds:

$$
\|v\|_{\mathrm{L}^{2}(\sigma)} \leq \sqrt{10} \frac{1}{d_{\boldsymbol{x}_{c}, \sigma}^{1 / 2}}\left[\|v\|_{\mathrm{L}^{2}\left(D_{\sigma, 1 / 2}\right)}+h_{\sigma}|\nabla v|_{\mathrm{L}^{2}\left(D_{\sigma, 1 / 2}\right)}\right]
$$

where $h_{\sigma}=\operatorname{diam}(\sigma)+d_{\boldsymbol{x}_{\mathrm{c}}, \sigma}$.

Proof. Let $\sigma$ and $\boldsymbol{x}_{\mathrm{c}}$ be given, and $v$ be a function of $\mathrm{H}^{1}\left(D_{\sigma, 1 / 2}\right)$. Without loss of generality, we suppose that $\sigma$ is a part of the plane $\boldsymbol{x}^{(1)}=0$ and that $\boldsymbol{x}_{\mathrm{c}}$ is located at $\left(-d_{\boldsymbol{x}_{\mathrm{c}}, \sigma}, 0,0\right)^{t}$. Let us define the following mapping:

$$
F: \quad \mid \begin{array}{lll}
{[0,1] \times \sigma} & \rightarrow & D_{\sigma, 1} \\
(t, y) & \mapsto & x=t \boldsymbol{y}+(1-t) \boldsymbol{x}_{\mathrm{c}}
\end{array}
$$

where $D_{\sigma, 1}$ is the range of $F$ (see Fig. 7). This mapping is regular and we have: $\mathrm{d} \boldsymbol{x}=t^{2} d_{\boldsymbol{x}_{\mathrm{c}}, \sigma} \mathrm{d} t \mathrm{~d} \gamma(\boldsymbol{y})$. In addition, the following elementary geometrical relation holds:

$$
t=\frac{\boldsymbol{x}^{(1)}+d_{\boldsymbol{x}_{\mathrm{c}}, \sigma}}{d_{\boldsymbol{x}_{\mathrm{c}}, \sigma}}, \quad \text { and } \quad \mathrm{d} t \mathrm{~d} \gamma(\boldsymbol{y})=\frac{d_{\boldsymbol{x}_{\mathrm{c}}, \sigma}}{\left(\boldsymbol{x}^{(1)}+d_{\boldsymbol{x}_{\mathrm{c}}, \sigma}\right)^{2}} \mathrm{~d} \boldsymbol{x} .
$$




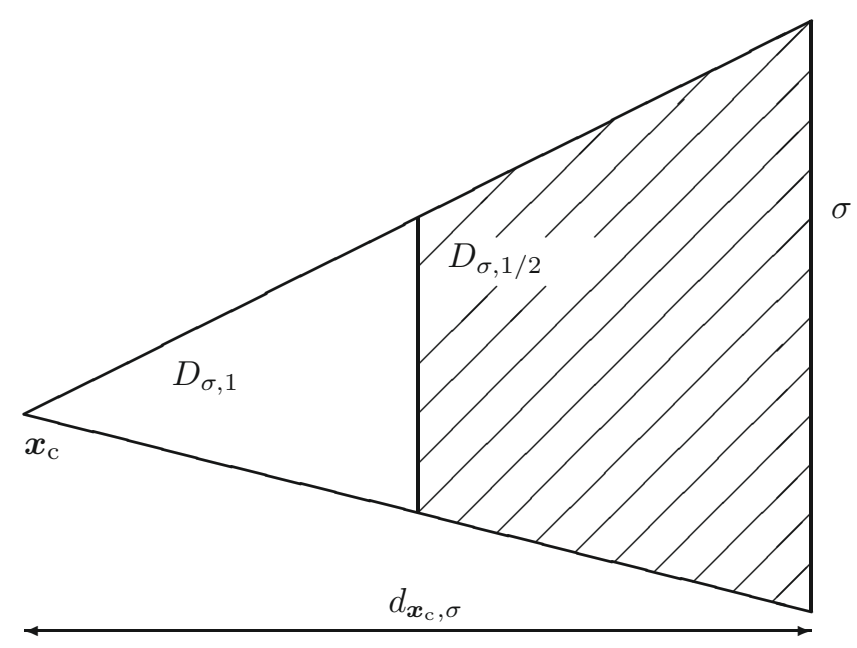

FiguRE 7. Sketch of the geometrical configuration used for Lemma A.1.

For any $\boldsymbol{y}$ on $\sigma$, we have:

$$
\begin{aligned}
v(\boldsymbol{y})^{2} & =\int_{1 / 2}^{1} \frac{\partial}{\partial t}\left[(2 t-1) v^{2}(F(t, \boldsymbol{y}))\right] \mathrm{d} t \\
& =2 \int_{1 / 2}^{1} v^{2}(F(t, \boldsymbol{y})) \mathrm{d} t+2 \int_{1 / 2}^{1}(2 t-1)\left[\boldsymbol{\nabla} v(F(t, \boldsymbol{y})) \cdot\left(\boldsymbol{y}-\boldsymbol{x}_{\mathrm{c}}\right)\right] v(F(t, \boldsymbol{y})) \mathrm{d} t .
\end{aligned}
$$

Integrating on $\sigma$, we thus get:

$$
\begin{aligned}
\int_{\sigma} v(\boldsymbol{y})^{2} \mathrm{~d} \gamma(\boldsymbol{y})= & \underbrace{2 \int_{\sigma} \int_{1 / 2}^{1} v^{2}(F(t, \boldsymbol{y})) \mathrm{d} t \mathrm{~d} \gamma(\boldsymbol{y})}_{T_{1}} \\
& +\underbrace{2 \int_{\sigma} \int_{1 / 2}^{1}(2 t-1)\left[\boldsymbol{\nabla} v(F(t, \boldsymbol{y})) \cdot\left(\boldsymbol{y}-\boldsymbol{x}_{\mathrm{c}}\right)\right] v(F(t, \boldsymbol{y})) \mathrm{d} t \mathrm{~d} \gamma(\boldsymbol{y})}_{T_{2}} .
\end{aligned}
$$

On one hand, the first term of this relation can be estimated as follows:

$$
T_{1}=2 \int_{D_{\sigma, 1 / 2}} v(\boldsymbol{x})^{2} \frac{d_{\boldsymbol{x}_{\mathrm{c}}, \sigma}}{\left(\boldsymbol{x}^{(1)}+d_{\boldsymbol{x}_{\mathrm{c}}, \sigma}\right)^{2}} \mathrm{~d} \boldsymbol{x} .
$$

As $\forall \boldsymbol{x} \in D_{\sigma, 1 / 2}, \boldsymbol{x}^{(1)} \geq-d_{\boldsymbol{x}_{\mathrm{c}}, \sigma} / 2$, the following inequality holds:

$$
\forall \boldsymbol{x} \in D_{\sigma, 1 / 2}, \quad \frac{d_{\boldsymbol{x}_{\mathrm{c}}, \sigma}}{\left(\boldsymbol{x}^{(1)}+d_{\boldsymbol{x}_{\mathrm{c}}, \sigma}\right)^{2}} \leq \frac{4}{d_{\boldsymbol{x}_{\mathrm{c}}, \sigma}}
$$

and thus:

$$
\left|T_{1}\right| \leq \frac{8}{d_{\boldsymbol{x}_{\mathrm{c}}, \sigma}}\|v\|_{\mathrm{L}^{2}\left(D_{\sigma, 1 / 2}\right)}^{2}
$$


On the second hand, as $\forall \boldsymbol{x} \in D_{\sigma, 1 / 2},\left\|\boldsymbol{x}-\boldsymbol{x}_{\mathrm{c}}\right\| \leq \operatorname{diam}(\sigma)+d_{\boldsymbol{x}_{\mathrm{c}}, \sigma}=h_{\sigma}$, the term $T_{2}$ can be bounded by:

$$
\begin{aligned}
\left|T_{2}\right| & \leq 2 h_{\sigma} \int_{\sigma} \int_{1 / 2}^{1}\|\nabla v(F(t, \boldsymbol{y}))\||v(F(t, \boldsymbol{y}))| \mathrm{d} t \mathrm{~d} \gamma(\boldsymbol{y}) \\
& =2 h_{\sigma} \int_{D_{\sigma, 1 / 2}}\|\boldsymbol{\nabla} v(\boldsymbol{x})\||v(\boldsymbol{x})| \frac{d_{\boldsymbol{x}_{\mathrm{c}}, \sigma}}{\left(\boldsymbol{x}^{(1)}+d_{\boldsymbol{x}_{\mathrm{c}}, \sigma}\right)^{2}} \mathrm{~d} \boldsymbol{x} \\
& \leq \frac{8}{d_{\boldsymbol{x}_{\mathrm{c}}, \sigma}} h_{\sigma}\|\boldsymbol{\nabla} v\|_{\mathrm{L}^{2}\left(D_{\sigma, 1 / 2}\right)}\|v\|_{\mathrm{L}^{2}\left(D_{\sigma, 1 / 2}\right)} .
\end{aligned}
$$

Collecting the bounds for $T_{1}$ and $T_{2}$, we get:

$$
\|v\|_{\mathrm{L}^{2}(\sigma)}^{2} \leq \frac{8}{d_{\boldsymbol{x}_{c}, \sigma}}\left[\|v\|_{\mathrm{L}^{2}\left(D_{\sigma, 1 / 2}\right)}^{2}+h_{\sigma}\|\nabla v\|_{\mathrm{L}^{2}\left(D_{\sigma, 1 / 2}\right)}\|v\|_{\mathrm{L}^{2}\left(D_{\sigma, 1 / 2}\right)}\right]
$$

and thus, thanks to Young's inequality, we obtain $\forall \alpha>0$ :

$$
\|v\|_{\mathrm{L}^{2}(\sigma)}^{2} \leq \frac{8}{d_{\boldsymbol{x}_{\mathrm{c}}, \sigma}}\left[\left(1+\frac{\alpha}{2}\right)\|v\|_{\mathrm{L}^{2}\left(D_{\sigma, 1 / 2}\right)}^{2}+h_{\sigma}^{2} \frac{1}{2 \alpha}\|\nabla v\|_{\mathrm{L}^{2}\left(D_{\sigma, 1 / 2}\right)}^{2}\right] .
$$

Choosing $\alpha=1 / 2$ yields the result.

In two dimensions, extending to this case the preceding notations, the following similar estimate is proven in $[24]$ :

$$
\|v\|_{\mathrm{L}^{2}(\sigma)} \leq \sqrt{2}\left(\frac{m_{\sigma}}{m_{D_{\sigma, 1}}}\right)^{1 / 2}\left[\|v\|_{\mathrm{L}^{2}\left(D_{\sigma, 1}\right)}+h_{\sigma}|\nabla v|_{\mathrm{L}^{2}\left(D_{\sigma, 1}\right)}\right] .
$$

We recall the following Poincaré inequality, proved by Payne and Weinberger [21] and valid for any convex domain $\omega$ :

$$
\forall \phi \in \mathrm{H}^{1}(\omega) \text { such that } \int_{\omega} \phi(\boldsymbol{x}) \mathrm{d} \boldsymbol{x}=0, \quad\|\phi\|_{\mathrm{L}^{2}(\omega)} \leq \frac{\operatorname{diam}(\omega)}{\pi}|\phi|_{\mathrm{H}^{1}(\omega)} .
$$

We are now in position to give a bound of elementary (i.e. related to a single edge or control volume) consistency residuals; this is the aim of the following two lemmas.

Lemma A.2. We suppose that the assumption (2.2) holds. Let $\mathcal{D}$ be an admissible discretization of $\Omega$ in the sense of Definition 3.1 and let $\theta>0$ be such that $\operatorname{regul}(\mathcal{D})>\theta$. Let $v$ be a function of $\mathrm{H}^{2}(\Omega) \cap \mathrm{H}_{0}^{1}(\Omega)$. Then the following bounds hold:

$$
\begin{array}{ll}
\forall \sigma \in\left(\mathcal{E}_{\text {int }} \cup \mathcal{E}_{\text {ext }}\right), & \left|R_{\Delta, \sigma}(v)\right| \leq c_{\Delta} \frac{\left[\sum_{K} \text { s.t. } \sigma \in \mathcal{E}(K)\right.}{m_{\sigma}^{1 / 2} \mathrm{~d}_{\sigma}^{3 / 2}} h_{K}^{2} \\
\forall K \in \mathcal{M}, & \left|R_{\mathrm{o}, K}(v)\right| \leq c_{\mathrm{o}} h_{K} m_{K}^{-1 / 2}\|v\|_{\mathrm{H}^{2}\left(D_{\sigma}\right)}
\end{array}
$$

where the positive real numbers $c_{\Delta}$ and $c_{\mathrm{o}}$ only depends on $d$ and $\theta$. In addition, if the mesh is super-admissible in the sense of Definition 3.2, we have:

$$
\forall \sigma \in \mathcal{E}_{\text {int }}, \sigma=\left.K|L \quad| R_{\mathrm{div}, \sigma}(v)\left|\leq c_{\operatorname{div}} \frac{\left[h_{K}+h_{L}\right]^{2}}{\left(m_{\sigma} \mathrm{d}_{\sigma}\right)^{1 / 2}}\right| v\right|_{\mathrm{H}^{2}\left(D_{\sigma}\right)}
$$

where the positive real number $c_{\mathrm{div}}$ only depends on $d$ and $\theta$. 
Proof. By definition of $R_{\Delta, \sigma}(v)$, the bound (A.4) is proven in [11], pp. 786-789, and a stronger version of the estimate (A.5) is given in [18], Lemma 3.3, equation (3.26). We now turn to the proof of (A.6). By definition of $R_{\mathrm{div}, \sigma}$, this bound is equivalent to:

$$
\left|\frac{d_{L, \sigma}}{d_{\sigma}} v\left(\boldsymbol{x}_{K}\right)+\frac{d_{K, \sigma}}{d_{\sigma}} v\left(\boldsymbol{x}_{L}\right)-\frac{1}{m_{\sigma}} \int_{\sigma} v(\boldsymbol{x}) \mathrm{d} \gamma(\boldsymbol{x})\right| \leq c_{\mathrm{div}} \frac{\left[h_{K}+h_{L}\right]^{2}}{\left(m_{\sigma} \mathrm{d}_{\sigma}\right)^{1 / 2}}|v|_{\mathrm{H}^{2}\left(D_{\sigma}\right)} .
$$

Let $v$ be now a function of $\mathrm{C}^{2}\left(\overline{D_{\sigma}}\right)$. The two following Taylor expansions hold for any $\boldsymbol{x}$ of $\sigma=K \mid L$ :

$$
\begin{aligned}
& v\left(\boldsymbol{x}_{K}\right)=v(\boldsymbol{x})+\boldsymbol{\nabla} v(\boldsymbol{x}) \cdot\left(\boldsymbol{x}_{K}-\boldsymbol{x}\right)+\int_{0}^{1}\left[\boldsymbol{H}(v)\left(t \boldsymbol{x}+(1-t) \boldsymbol{x}_{K}\right)\left(\boldsymbol{x}_{K}-\boldsymbol{x}\right)\right] \cdot\left(\boldsymbol{x}_{K}-\boldsymbol{x}\right) t \mathrm{~d} t \\
& v\left(\boldsymbol{x}_{L}\right)=v(\boldsymbol{x})+\boldsymbol{\nabla} v(\boldsymbol{x}) \cdot\left(\boldsymbol{x}_{L}-\boldsymbol{x}\right)+\int_{0}^{1}\left[\boldsymbol{H}(v)\left(t \boldsymbol{x}+(1-t) \boldsymbol{x}_{L}\right)\left(\boldsymbol{x}_{L}-\boldsymbol{x}\right)\right] \cdot\left(\boldsymbol{x}_{L}-\boldsymbol{x}\right) t \mathrm{~d} t
\end{aligned}
$$

where $\boldsymbol{H}(v)(\boldsymbol{x})$ is the Hessian matrix of $v$ at point $\boldsymbol{x}$.

Multiplying the first relation by $d_{L, \sigma} / d_{\sigma}$, the second one by $d_{K, \sigma} / d_{\sigma}$, summing, integrating over $\sigma$ and dividing by $m_{\sigma}$ yields:

$$
\frac{d_{L, \sigma}}{d_{\sigma}} v\left(\boldsymbol{x}_{K}\right)+\frac{d_{K, \sigma}}{d_{\sigma}} v\left(\boldsymbol{x}_{L}\right)=\frac{1}{m_{\sigma}} \int_{\sigma} v(\boldsymbol{x}) \mathrm{d} \gamma(\boldsymbol{x})+\underbrace{\frac{1}{m_{\sigma}} \int_{\sigma} \boldsymbol{\nabla} v(\boldsymbol{x}) \cdot\left(\boldsymbol{x}_{G}-\boldsymbol{x}\right) \mathrm{d} \gamma(\boldsymbol{x})}_{T_{1}}+\frac{d_{L, \sigma}}{d_{\sigma}} R_{K, \sigma}+\frac{d_{K, \sigma}}{d_{\sigma}} R_{L, \sigma}
$$

where $\boldsymbol{x}_{G}=\frac{d_{L, \sigma}}{d_{\sigma}} \boldsymbol{x}_{K}+\frac{d_{K, \sigma}}{d_{\sigma}} \boldsymbol{x}_{L}$ is the mass center of $\sigma$, thanks to the fact that the mesh is assumed to be super-admissible, and:

$$
\begin{aligned}
& R_{K, \sigma}=\frac{1}{m_{\sigma}} \int_{\sigma} \int_{0}^{1}\left[\boldsymbol{H}(v)\left(t \boldsymbol{x}+(1-t) \boldsymbol{x}_{K}\right)\left(\boldsymbol{x}_{K}-\boldsymbol{x}\right)\right] \cdot\left(\boldsymbol{x}_{K}-\boldsymbol{x}\right) t \mathrm{~d} t \mathrm{~d} \gamma(\boldsymbol{x}) \\
& R_{L, \sigma}=\frac{1}{m_{\sigma}} \int_{\sigma} \int_{0}^{1}\left[\boldsymbol{H}(v)\left(t \boldsymbol{x}+(1-t) \boldsymbol{x}_{L}\right)\left(\boldsymbol{x}_{L}-\boldsymbol{x}\right)\right] \cdot\left(\boldsymbol{x}_{L}-\boldsymbol{x}\right) t \mathrm{~d} t \mathrm{~d} \gamma(\boldsymbol{x}) .
\end{aligned}
$$

The following bound of these quantities is given in [11], pp. 786-789:

$$
\left|R_{K, \sigma}\right| \leq c_{1} \frac{h_{K}^{2}}{\left(m_{\sigma} d_{K, \sigma}\right)^{1 / 2}}|v|_{\mathrm{H}^{2}\left(D_{K, \sigma}\right)}, \quad\left|R_{L, \sigma}\right| \leq c_{1} \frac{h_{L}^{2}}{\left(m_{\sigma} d_{L, \sigma}\right)^{1 / 2}}|v|_{\mathrm{H}^{2}\left(D_{L, \sigma}\right)}
$$

where $c_{1}$ only depends on the space dimension $d$. On the other hand, we have:

$$
T_{1}=\frac{1}{m_{\sigma}} \int_{\sigma} \boldsymbol{\nabla} v(\boldsymbol{x}) \cdot\left(\boldsymbol{x}_{G}-\boldsymbol{x}\right) \mathrm{d} \gamma(\boldsymbol{x})=\frac{1}{m_{\sigma}} \int_{\sigma} \boldsymbol{\nabla}(v(\boldsymbol{x})-p(\boldsymbol{x})) \cdot\left(\boldsymbol{x}_{G}-\boldsymbol{x}\right) \mathrm{d} \gamma(\boldsymbol{x})
$$

for any linear polynomial $p(\cdot)$. Using the Cauchy-Schwarz inequality then the convenient trace lemma, i.e. choosing either $K$ or $L$ and applying (A.2) for $d=2$ and (A.1) for $d=3$, both with $\boldsymbol{x}_{\mathrm{c}}$ equal to $\boldsymbol{x}_{K}$ or $\boldsymbol{x}_{L}$, we obtain:

$$
\begin{aligned}
\left|T_{1}\right| & \leq \frac{\max \left(h_{K}, h_{L}\right)}{m_{\sigma}^{1 / 2}}\|\nabla(v-p)\|_{\mathrm{L}^{2}(\sigma)} \\
& \leq c_{2} \frac{\max \left(h_{K}, h_{L}\right)}{m_{\sigma}^{1 / 2}} \frac{1}{\max \left(d_{K, \sigma}, d_{L, \sigma}\right)^{1 / 2}}\left[\|\nabla(v-p)\|_{\mathrm{L}^{2}\left(D_{\sigma}\right)}+\max \left(h_{K}, h_{L}\right)|v|_{\mathrm{H}^{2}\left(D_{\sigma}\right)}\right]
\end{aligned}
$$


where $c_{2}$ only depends on the regularity of the mesh. Choosing for $p(\cdot)$ the function defined by:

$$
p(\boldsymbol{x})=\sum_{i=1}^{d} \boldsymbol{x}^{(i)} \frac{1}{m_{D_{\sigma}}} \int_{D_{\sigma}} \frac{\partial v}{\partial \boldsymbol{x}^{(i)}}(\boldsymbol{x}) \mathrm{d} \boldsymbol{x}
$$

and applying the Poincaré inequality (A.3) concludes the proof.

Lemma A.3. We suppose that hypotheses (2.2) holds. Let $\mathcal{D}$ be an admissible discretization of $\Omega$ in the sense of Definition 3.1 and $\theta>0$ be such that $\operatorname{regul}(\mathcal{D})>\theta$. Let $v \in \mathrm{H}^{1}(\Omega)$. Then, for each edge $\sigma$ of the mesh and each control volume $K$ such that $\sigma \subset \bar{K}$, we have:

$$
\left|\frac{1}{m_{\sigma}} \int_{\sigma} v(\boldsymbol{x}) \mathrm{d} \gamma(\boldsymbol{x})-P_{\mathcal{M}}(v)_{K}\right| \leq c\left[\frac{h_{K}}{m_{\sigma}}\right]^{1 / 2}|v|_{\mathrm{H}^{1}(K)}
$$

where $c$ only depends on $d$ and $\theta$. Consequently, for each pair of neighbouring control volumes $K$ and $L$ of the mesh, the following estimate holds:

$$
\left|P_{\mathcal{M}}(v)_{L}-P_{\mathcal{M}}(v)_{K}\right| \leq 2 c\left[\frac{h_{K}+h_{L}}{m_{K \mid L}}\right]^{1 / 2}|v|_{\mathrm{H}^{1}(K \cup L)} .
$$

Proof. The results (A.8)-(A.9) are proven for $d=2$ in [11], pp. 777-779. We provide here a proof of this latter estimate valid for $d=2$ and $d=3$. Let $\sigma$ be an edge of the mesh, $K$ one control volume such that $\sigma \subset \bar{K}$ and $v$ a function of $\mathrm{H}^{1}(K)$. We have:

$$
R=P_{\mathcal{M}}(v)_{K}-\frac{1}{m_{\sigma}} \int_{\sigma} v(\boldsymbol{x}) \mathrm{d} \gamma(\boldsymbol{x})=\frac{1}{m_{\sigma}} \int_{\sigma}\left(P_{\mathcal{M}}(v)_{K}-v(\boldsymbol{x})\right) \mathrm{d} \gamma(\boldsymbol{x})
$$

By the Cauchy-Schwarz inequality then the estimates (A.2) (for $d=2$ ) or (A.1) (for $d=3$ ) with $\boldsymbol{x}_{\mathrm{c}}=\boldsymbol{x}_{K}$, we get:

$$
|R| \leq \frac{1}{m_{\sigma}^{1 / 2}}\left\|v-P_{\mathcal{M}}(v)_{K}\right\|_{\mathrm{L}^{2}(\sigma)} \leq c_{1} \frac{1}{\left(m_{\sigma} d_{K, \sigma}\right)^{1 / 2}}\left[\left\|v-P_{\mathcal{M}}(v)_{K}\right\|_{\mathrm{L}^{2}(K)}+h_{K}\|\nabla v\|_{\mathrm{L}^{2}(K)}\right]
$$

and thus, by the Poincaré inequality (A.3):

$$
|R| \leq c_{2} \frac{h_{K}}{\left(m_{\sigma} d_{K, \sigma}\right)^{1 / 2}}\|\nabla v\|_{\mathrm{L}^{2}(K)}
$$

where both $c_{1}$ and $c_{2}$ only depends on $d$. The estimate (A.8) follows using regularity assumptions for the mesh; inequality (A.9) is then an easy consequence of this result and the triangle inequality.

Corollary A.4. We suppose that hypotheses (2.2) holds. Let $\mathcal{D}$ be an admissible discretization of $\Omega$ in the sense of Definition 3.1 and $\theta>0$ be such that $\operatorname{regul}(\mathcal{D}, \mathcal{G})>\theta$. Let $v \in \mathrm{H}^{1}(\Omega)$. Then the following bounds hold:

$$
\begin{array}{ll}
\forall \sigma \in\left(\mathcal{E}_{\text {int }} \cup \mathcal{E}_{\text {ext }}\right), & \left|R_{\mathrm{grad}, \mathcal{M}, \sigma}\right| \leq c_{\mathrm{g}, \mathcal{M}} \frac{\left[\sum_{K} h_{\text {s.t. } \sigma \in \mathcal{E}(K)} h_{K}\right]^{1 / 2}}{m_{\sigma}^{1 / 2}}|v|_{\mathrm{H}^{1}\left(\cup_{K} \text { s.t. } \sigma \in \mathcal{E}(K)\right.}{ }^{K)}( \\
\forall \sigma \in \mathcal{E}_{\text {int }}, \sigma=K \mid L & \left|R_{\mathrm{stab}, \mathcal{M}, \sigma}\right| \leq c_{\mathrm{stab}} \frac{\left[h_{K}+h_{L}\right]^{3 / 2}}{m_{\sigma}^{1 / 2}}|v|_{\mathrm{H}^{1}(K \cup L)} \\
\forall \sigma \in\left(\mathcal{E}_{\text {int }} \cup \mathcal{E}_{\text {ext }}\right), & \left|R_{\mathrm{grad}, \mathcal{G}, \sigma}\right| \leq c_{\mathrm{g}, \mathcal{G}}\left[\max _{K \in\left(G_{\sigma}\right)}\left(h_{K}\right) \frac{1}{m_{\sigma}}\right]^{1 / 2}|v|_{\mathrm{H}^{1}\left(C_{\sigma}\right)}
\end{array}
$$


where the three positive real numbers $c_{\mathrm{g}, \mathcal{M}}, c_{\mathrm{stab}}$ and $c_{\mathrm{g}, \mathcal{G}}$ only depend on $d$ and $\theta$ and, in the last relation, $G_{\sigma}$ and $C_{\sigma}$ are defined as follows:

- if $\sigma \in \mathcal{E}_{\text {int }}, \sigma=K \mid L$, then $G_{\sigma}=G_{K} \cup G_{L}$ and $C_{\sigma}=\cup_{M \in\left(G_{K} \cup G_{L}\right)} M$;

- if $\sigma \in \mathcal{E}_{\text {ext }}, \sigma \in \mathcal{E}(K), G_{\sigma}=G_{K}$ and $C_{\sigma}=\cup_{M \in G_{K}} M$.

Note that, if $\sigma$ is an internal edge of a cluster, $G_{K}=G_{L}$.

Proof. The estimate (A.10) is the same relation as (A.8) for an external edge or (A.9) for an internal one. The bound (A.11) follows directly from (A.9). Let us prove the estimate (A.12). Let $\sigma$ be an edge of the mesh and $K$ a control volume such that $\sigma \in \mathcal{E}(K)$. First, we suppose that $\sigma$ is an external edge of the mesh. By definition, we have:

$$
\left|R_{\text {grad }, \mathcal{G}, \sigma}\right|=\left|\frac{1}{\left(\sum_{L \in G_{K}} m_{L}\right)} \int_{\cup L \in G_{K}} v(\boldsymbol{x}) \mathrm{d} \boldsymbol{x}-\frac{1}{m_{\sigma}} \int_{\sigma} v(\boldsymbol{x}) \mathrm{d} \gamma(\boldsymbol{x})\right| .
$$

Decomposing the first term, we get:

$$
\begin{aligned}
\left|R_{\mathrm{grad}, \mathcal{G}, \sigma}\right|= & \left|\sum_{L \in G_{K}} \frac{m_{L}}{\left(\sum_{L \in G_{K}} m_{L}\right)} \frac{1}{m_{L}} \int_{L} v(\boldsymbol{x}) \mathrm{d} \boldsymbol{x}-\frac{1}{m_{\sigma}} \int_{\sigma} v(\boldsymbol{x}) \mathrm{d} \gamma(\boldsymbol{x})\right| \\
= & \mid \sum_{L \in G_{K}} \frac{m_{L}}{\left(\sum_{L \in G_{K}} m_{L}\right)}[\underbrace{\frac{1}{m_{L}} \int_{L} v(\boldsymbol{x}) \mathrm{d} \boldsymbol{x}-\frac{1}{m_{K}} \int_{K} v(\boldsymbol{x}) \mathrm{d} \boldsymbol{x}}_{T_{K, L}}] \\
& +\underbrace{\frac{1}{m_{K}} \int_{K} v(\boldsymbol{x}) \mathrm{d} \boldsymbol{x}-\frac{1}{m_{\sigma}} \int_{\sigma} v(\boldsymbol{x}) \mathrm{d} \gamma(\boldsymbol{x})}_{T_{2}} \mid
\end{aligned}
$$

The latter difference $T_{2}$ is bounded by (A.8), and, if $K$ can be chosen such that, for each $L$ in $G_{K}, K$ and $L$ are two neighbouring control volumes, each difference $T_{K, L}$ in the sum is bounded by (A.9); otherwise, this difference may be decomposed in a sum of differences of the mean value of $v(\cdot)$ over two neighbouring control volumes, the number of terms of this sum being bounded by the number of control volumes in the cluster $G_{K}$. This completes the proof of (A.12), in the case where $\sigma \in \mathcal{E}_{\text {ext }}$. When $\sigma$ is an internal edge of a cluster, the quantities $P_{\mathcal{G}}(v)_{K}$ and $P_{\mathcal{G}}(v)_{L}$ are identical, and the definition of $R_{\operatorname{grad}, \mathcal{G}, \sigma}$ is the same as in the previous case. Finally, when $\sigma$ is at the boundary of two clusters, the bound (A.12) follows from the same argument, using the triangle inequality.

Acknowledgements. The authors would like to thank an anonymous referee for an helpful suggestion which significantly improved the contents of this paper.

\section{REFERENCES}

[1] F. Archambeau, N. Méchitoua and M. Sakiz, Code saturne: A finite volume code for turbulent flows. International Journal of Finite Volumes 1 (2004), http://www.latp.univ-mrs.fr/IJFV/.

[2] M. Bern, D. Eppstein and J. Gilbert, Provably good mesh generation. J. Comput. System Sci. 48 (1994) 384-409.

[3] F. Boyer and P. Fabrie, Eléments d'analyse pour l'étude de quelques modèles d'écoulements de fluides visqueux incompressibles, Mathématiques et Applications 52. Springer-Verlag (2006). 
[4] F. Brezzi and M. Fortin, A minimal stabilisation procedure for mixed finite element methods. Numer. Math. 89 (2001) $457-491$.

[5] E. Chénier, R. Eymard and O. Touazi, Numerical results using a colocated finite-volume scheme on unstructured grids for incompressible fluid flows. Numer. Heat Transf. Part B: Fundam. 49 (2006) 259-276.

[6] E. Chénier, R. Eymard, R. Herbin and O. Touazi, Collocated finite volume schemes for the simulation of natural convective flows on unstructured meshes. Int. J. Num. Methods Fluids 56 (2008) 2045-2068.

[7] Y. Coudière, T. Gallouët and R. Herbin, Discrete Sobolev inequalities and $L^{p}$ error estimates for finite volume solutions of convection diffusion equations. ESAIM: M2AN 35 (2001) 767-778.

[8] K. Deimling, Nonlinear functional analysis. Springer-Verlag (1985).

[9] R. Eymard and T. Gallouët, H-convergence and numerical schemes for elliptic equations. SIAM J. Numer. Anal. 41 (2003) 539-562.

[10] R. Eymard and R. Herbin, A new colocated finite volume scheme for the incompressible Navier-Stokes equations on general non-matching grids. C. R. Acad. Sci., Sér. I Math. 344 (2007) 659-662.

[11] R. Eymard, T. Gallouët and R. Herbin, Finite volume methods, Handbook of Numerical Analysis VII. North Holland (2000) $713-1020$.

[12] R. Eymard, T. Gallouët and R. Herbin, A finite volume scheme for anisotropic diffusion problems. C. R. Acad. Sci., Sér. I Math. 339 (2004) 299-302.

[13] R. Eymard, R. Herbin and J.C. Latché, On a stabilized colocated finite volume scheme for the Stokes problem. ESAIM: M2AN 40 (2006) 501-528.

[14] R. Eymard, T. Gallouët, R. Herbin and J.-C. Latché, Analysis tools for finite volume schemes. Acta Mathematica Universitatis Comenianae 76 (2007) 111-136.

[15] R. Eymard, R. Herbin and J.C. Latché, Convergence analysis of a colocated finite volume scheme for the incompressible Navier-Stokes equations on general 2D or 3D meshes. SIAM J. Numer. Anal. 45 (2007) 1-36.

[16] R. Eymard, R. Herbin, J.C. Latché and B. Piar, On the stability of colocated clustered finite volume simplicial discretizations for the 2D Stokes problem. Calcolo 44 (2007) 219-234.

[17] L.P. Franca and R. Stenberg, Error analysis of some Galerkin Least Squares methods for the elasticity equations. SIAM J. Numer. Anal. 28 (1991) 1680-1697.

[18] T. Gallouët, R. Herbin and M.H. Vignal, Error estimates for the approximate finite volume solution of convection diffusion equations with general boundary conditions. SIAM J. Numer. Anal. 37 (2000) 1935-1972.

[19] V. Girault and P.-A. Raviart, Finite Element Methods for Navier-Stokes Equations - Theory and Algorithms, Springer Series in Computational Mathematics 5. Springer-Verlag (1986).

[20] J. Nečas, Équations aux dérivées partielles. Presses de l'Université de Montréal (1965).

[21] L.E. Payne and H.F. Weinberger, An optimal Poincaré-inequality for convex domains. Arch. Rational Mech. Anal. 5 (1960) 286-292.

[22] B. Piar, PELICANS : Un outil d'implémentation de solveurs d'équations aux dérivées partielles. Note Technique 2004/33, IRSN/DPAM/SEMIC (2004).

[23] R. Temam, Navier-Stokes Equations, Studies in mathematics and its applications. North-Holland (1977).

[24] R. Verfürth, Error estimates for some quasi-interpolation operators. ESAIM: M2AN 33 (1999) 695-713. 